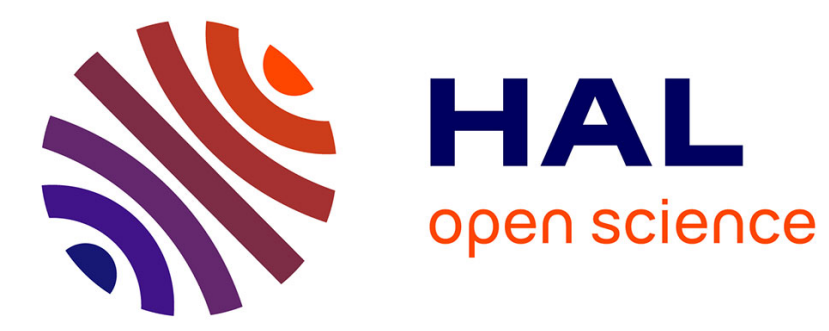

\title{
SELF-INDUCED SYNCHRONIZATION BY LARGE DELAY
}

Camille Poignard

\section{To cite this version:}

Camille Poignard. SELF-INDUCED SYNCHRONIZATION BY LARGE DELAY. 2021. hal03183760

\author{
HAL Id: hal-03183760 \\ https://hal.science/hal-03183760
}

Preprint submitted on 28 Mar 2021

HAL is a multi-disciplinary open access archive for the deposit and dissemination of scientific research documents, whether they are published or not. The documents may come from teaching and research institutions in France or abroad, or from public or private research centers.
L'archive ouverte pluridisciplinaire HAL, est destinée au dépôt et à la diffusion de documents scientifiques de niveau recherche, publiés ou non, émanant des établissements d'enseignement et de recherche français ou étrangers, des laboratoires publics ou privés. 


\title{
SELF-INDUCED SYNCHRONIZATION BY LARGE DELAY
}

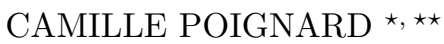 \\ * EPSRC Centre for Predictive Modelling in Healthcare, University of Exeter, Exeter EX4 4QF, UK \\ ** Instituto de Ciências Matemáticas e Computação, Universidade de São Paulo, São Carlos 13566-590, \\ Brazil
}

\begin{abstract}
We investigate the dynamics of a delay differential equation obtained by perturbing a vector field $\mathrm{f}: \mathbb{R}^{n} \rightarrow \mathbb{R}^{n}$, admitting a stable periodic orbit, thanks to a delayed feedback control $\eta \mathrm{g}(x, x(t-\tau))$ of same regularity, where $\eta$ is small and $\tau$ large so that $\eta \tau$ be bounded but non small. We prove that trajectories starting in a neighborhood (of size independent on the parameters $\eta, \tau$ ) of this original periodic orbit in the set of continuous functions from $[-\tau, 0]$ to $\mathbb{R}^{n}$ enter asymptotically a periodic regime, which regimes existing in finite number. Our synchronization result is based on the construction of an invariant manifold via a process inspired by the Lyapunov-Perron method for integral operators associated to solutions of ordinary differential equations, and on the persistence of normally hyperbolic invariant manifolds for semi-flows on Banach spaces. The statement we provide here complements already known results on periodic orbits in the context of delay differential equations.
\end{abstract}

\section{INTRODUCTION}

The control of nonlinear dynamical systems is an active research area which has led to many improvements in engineering disciplines, notably for: driving multibody systems, stabilising spacecrafts attitude [25], controlling blood glucose [33] and neural firing rate means [26], stabilising erratic dynamics of synthetic gene circuits [39] (or inducing such behaviors [31]).

In this context, among the different methods of control developed, time-delayed feedbacks have now been used for several decades. Initiated at the end of the 1980s with the seminal work of Ott, Grebogi, and Yorke on chaos control [27], several delay feedbacks have been proved to be useful to turn an unstable periodic orbit of a chaotic attractor into a stable one: a particular scheme, efficient and easy to implement in practical experiments, is the control of Pyragas [32], who introduced a feedback term given by the difference between the dynamical state of a signal and its value delayed by a time equal to the period of this signal.

Since these two pioneering works, various investigations have been performed successfully in this direction, for instance to stabilize an unstable equilibrium point ([20], [43]), to control the synchronization of dynamical networks [21], to stabilize unstable periodic orbits emerging from a subcritical Hopf bifurcation ([6], [13]), or again rotating waves near a fold bifurcation [14]. All these investigations led to new insights and achievements for various fields of applications, including the control of stochastic neural synchrony in the brain [34] (suspected to be responsible of disease such as Parkinson or epilepsy) among other therapeutic approaches, and the stabilization of electrochemical or optoelectronic systems ([10], [40], [14], [15]), in control engineering. The genericity of stabilization by such delayed feedback controls has also been tackled, showing that, in principle, there is no restriction for their use (see [37]).

In this paper, we consider the perturbation of a vector field $\mathrm{f}: \mathbb{R}^{n} \rightarrow \mathbb{R}^{n}$ (admitting a stable periodic orbit $\Gamma$ in $\mathbb{R}^{n}$ ) by a delay feedback control of the form $\eta \mathrm{g}(x(t), x(t-\tau))$. Compared to the studies mentioned above, we are not interested in turning $\Gamma$ into an unstable orbit, but our goal is to describe the dynamical phenomena induced by the delay $\tau$, and this in a neighborhood of $\Gamma$ in the infinite-dimensional phase space corresponding to this type of perturbation. In particular, we are interested in the following questions: how much of periodicity persists or is enhanced thanks to the delay feedback? Does the delay induce other type of

Date: 1 February 2020. 
phenomena (including chaos or other complicated dynamics which can occur even for scalar delay dynamical systems [18]) in such a neighborhood?

This idea of looking at the emergence of particular dynamical states from the delay is not at all new. In fact, Pyragas himself had suggested the existence of a delay-induced multistability in his famous article. Since then, a work done by Perlikowskwi and Yanchuk in [28] (followed later by Sieber, Wolfrum, Lichtner and Yanchuk in [38]) has provided some answers to the questions above: they showed that given a smooth delay equation $\dot{x}=\mathrm{f}(x(t), x(t-\tau))$ admitting a periodic orbit, then the presence of this orbit automatically implies its reappearance when the delay is increasing: as a consequence, they deduced that more and more periodic orbits appear when the delay tends to infinity, some of them stable, others, unstable. This is in accordance with experiments observed in lasers when the delay taken is large ([42], [23]).

In this context, our purpose here is to prove that for our delay equation $\dot{x}=\mathrm{f}(x)+\eta \mathrm{g}(x(t), x(t-\tau))$ taken when $\eta>0$ is small and $\tau>0$ large so that $\eta \tau$ be uniformly bounded but non small, there is nothing else but periodic orbits in a neighborhood (whose size does not depend on $\eta, \tau$ ) of the original orbit $\Gamma$ : we provide a Mathematical statement saying that, any trajectory in the phase space $\mathscr{C}\left([-\tau, 0], \mathbb{R}^{n}\right)$ starting in such a neighborhood converges asymptotically to a periodic orbit, these periodic orbits existing in finite number. Hence the trajectories in this neighborhood exhibit a delayed-induced synchronization, and they are split in clusters of synchronization with distinct frequencies: we give a formula (at first order in $\eta$ ) for these distinct frequencies. Moreover the larger $\tau$ is (at a fixed value of $\eta$ ), the more numerous are the periodic orbits, and thus the more numerous are the different clusters.

Our work therefore completes the one of Perlikowski and Yanchuk in [28], in the particular case of our equation with the two parameters $\eta, \tau$. Our result shows three main assets. The first one is to hold for a non trivial range of parameters, namely when the delay $\tau$ can possibly be very large, compared to classical perturbations results requiring $\tau$ to be fixed and $\eta$ small enough compared to $\tau$ (as a consequence $\eta \tau$ appears to be usually very small). The second one is to give a full description of the dynamics in a neighborhood of size fixed with respect to these parameters $\eta, \tau$ (which size depending only on the vector field f), and therefore in a neighborhood which is not very small. The third one is that this synchronization we obtained here is provided by a precise Mathematical statement valid for the delay equation $\dot{x}=\mathrm{f}(x)+\eta \mathrm{g}(x(t), x(t-\tau))$ itself with any (sufficiently smooth) maps $\mathrm{f}$ and $\mathrm{g}$, whereas, very often, such a result is established for phase reduction models deduced from such a delay equation (and, as said above, often in the situation where the delay is fixed or small: see [22] and very recently [1]). To our knowledge, these three points constitute a new result.

The approach we adopt to obtain it is based on the use of two classical tools in nonlinear dynamical systems theory. The first one is the Banach Contraction Mapping Principle [2], which, under the form of the Lyapunov-Perron method for curves solutions of an ordinary differential equation [29, 30, 9], has permitted to establish in the past the existence of various types of invariant manifolds on which it is convenient and usual to reduce such a dynamical system (see [35], [36], [41]): we adapt this method here for our delay system, and as a result obtain an averaged system for which we manage to prove the desired frequency-locking. The second tool is the persistence of normally hyperbolic invariant manifolds under perturbation of semi-flows generated by delay differential equations: this persistence under small perturbations is well known for flows in finite dimensional spaces (see [12], [19], [24] and as well [11]) and it has been extended at the end of the 1990s by Bates, Lu, Zeng in $[3,4,5]$, in the infinite dimensional setting of semi-flows on Banach spaces. ${ }^{1}{ }^{2}$ Thanks to this result, we obtain that the desired synchronization exists not only for our averaged system, but also for our original delay equation.

We now present our result in details (Section 2), before giving its proof in the remaining part of the paper (in Sections 3, 4, 5, 6, 7 and in the Appendix).

\section{Presentation of the Main Result}

Let $\dot{x}(t)=\mathrm{f}(x)$ be a differential equation defined by a vector field of class $\mathscr{C}^{3}$ in $\mathbb{R}^{n}$, for which exists a stable hyperbolic periodic orbit $\Gamma$ of period $T$ in $\mathbb{R}^{n}$. In this paper we consider a perturbation of this vector

\footnotetext{
${ }^{1}$ The Lyapunov-Perron method permits as well to obtain all these persistence results, both in finite or infinite dimension.

${ }^{2}$ We reproduce the precise statement of this result in the Appendix.
} 
field by a delayed feedback term:

$$
\dot{x}=\mathrm{f}(x)+\eta \mathrm{g}(x(t), x(t-\tau)),
$$

in which $\mathrm{g}: \mathbb{R}^{2 n} \rightarrow \mathbb{R}^{n}$ is of class $\mathscr{C}^{3}$ as well, $\eta$ is taken very small, and the delay $\tau>0$ is possibly very large: in particular we allow the product $\eta \tau$ to be bounded but non small, which constitutes a non trivial case of persistence of periodic behavior under small perturbation.

2.1. Notations. In all the paper, $\mathbb{R}$ will denote the set of real numbers taken with the absolute value $|\cdot|$, $\mathbb{R}_{+}$the set $\left[0,+\infty\left[\right.\right.$, and $\mathbb{R}_{-}$the set $\left.]-\infty, 0\right]$. We will denote by $\mathbb{S}^{1}$ the circle of length $2 \pi$.

$\mathbb{R}^{n}$ will be equipped with a fixed norm denoted indistinctly by $\|\cdot\|$ for any $n \geq 2$. For square matrices of size $n-1$, we will also denote by $\|\cdot\|$ the matrix norm induced by $\|\cdot\|$ on $\mathbb{R}^{n-1}$.

$\left(\mathscr{C}([-1,0], \mathbb{R}),\|\cdot\|_{\infty}\right)$ will denote the Banach space of continuous functions from the interval $[-1,0]$ to $\mathbb{R}$ equipped with the uniform norm defined by the relation $\|\mathrm{f}\|_{\infty}=\sup _{s \in[-1,0]}|\mathrm{f}(s)|$, and similarly for other spaces such as $\mathscr{C}\left([-\tau, 0], \mathbb{R}^{n}\right)$, or $\mathscr{C}\left([-\tau, 0], \mathbb{R}^{n-1}\right)$. The notation $\left(\mathcal{B}_{\delta_{0}}\left(\mathbb{R}_{-}, \mathbb{R}^{n-1}\right),\|\cdot\|_{\infty}\right)$ will stand for the Banach space of continuous functions from $\mathbb{R}_{-}$to $\mathbb{R}^{n-1}$ uniformly bounded by $\delta_{0}$. We will need to use as well weighted Banach spaces, mainly the space $\mathscr{C}_{\alpha}\left(\mathbb{R}_{-}, \mathbb{R}\right)$ of functions having an exponential growth of rate at most given by $\alpha>0$ :

$$
\mathscr{C}_{\alpha}\left(\mathbb{R}_{-}, \mathbb{R}\right)=\left\{f: \mathbb{R}_{-} \rightarrow \mathbb{R} \text { continuous, }\|f\|_{\alpha}<+\infty\right\}
$$

where the norm $\|\cdot\|_{\alpha}$ is defined by the relation $\|f\|_{\alpha}=\sup _{s \leq 0}|f(s)| e^{\alpha s}$.

For products of two Banach spaces (whether those are spaces of functions or not), the norm taken will be always the one given by the maximum of the two norms.

For the delay differential equation (1), we denote by $x(t, \gamma)$ the trajectory of (1) starting at the initial condition $\gamma \in \mathscr{C}\left([-\tau, 0], \mathbb{R}^{n}\right)$ and taken at time $t$. We will also need to consider the semi-flow $\left(\mathcal{T}_{t, \eta, \tau}\right)_{t \geq 0}$ associated to this differential equation, i.e the map from $\mathscr{C}\left([-\tau, 0], \mathbb{R}^{n}\right)$ to itself defined by:

$$
\forall \gamma \in \mathscr{C}\left([-\tau, 0], \mathbb{R}^{n}\right), \forall s \in[-\tau, 0]: \mathcal{T}_{t, \eta, \tau}(\gamma)(s)=x(t+s, \gamma)
$$

These notations $x(t, \gamma)$ and $\mathcal{T}_{t, \eta, \tau}(\gamma)(s)$ will be adapted in a straightforward way to other delay differential equations: for instance for the delay equation (3) written in normal coordinates (see Lemma 2.2), we will denote by $\mathcal{T}_{t, \eta, \tau}\left(\phi_{0}, y_{0}\right)$ the semi-flow associated to (3) taken at the initial condition $\left(\phi_{0}, y_{0}\right)$. When semiflows of different equations will be considered in the same context, the reference to the considered equation will be explicitly mentioned in the notations: in Section 6 for instance, $\mathcal{T}_{t, \eta, \tau}^{\text {av }}$ will stand for the semi-flow of the averaged equation (15), and similarly $\mathcal{T}_{(8), t, \eta, \tau}$ will denote the semi-flow of (8).

We refer the reader to [18] for an introduction to these basic notions on delay differential equations.

We will often use the notation $\mathrm{f}(\cdot)$ to denote the function $x \mapsto \mathrm{f}(x)$ when this notation will be convenient. For instance in Section 6 , in Proposition 6.3 with the function $\eta \tau \mathrm{G}\left(\cdot+\theta_{\tau}\right)$ from $\mathbb{R}$ to $\mathbb{R}$, and in Lemma 6.5 with the functions $\frac{2 \pi \tau}{T} \cdot+C_{1}$ from $\mathbb{R}_{-}$to $\mathbb{R}$ standing for $s \mapsto \frac{2 \pi \tau}{T} s+C_{1}$, and $\omega_{i}^{*} \cdot+C_{2}$ standing for $s \mapsto \omega_{i}^{*} s+C_{2}$. Similarly in other parts of the paper, notably in Corollary 7.1 with the function $\chi_{i, C}$ from $\mathbb{R}_{-}$to $\mathbb{R}$ written as:

$$
\chi_{i, C}=\frac{2 \pi \tau}{T}+\omega_{i}^{*} \cdot+C+\alpha_{i}(\cdot, \eta, \tau, C)+\beta_{i}(\cdot, \eta, \tau, C)
$$

and standing for:

$$
s \mapsto \frac{2 \pi \tau s}{T}+\omega_{i}^{*} s+C+\alpha_{i}(s, \eta, \tau, C)+\beta_{i}(s, \eta, \tau, C) .
$$

For a map F defined on $\mathbb{R}^{n-1}$ and of class $\mathscr{C}^{r}, r \geq 1$, the notation $D \mathrm{~F}(z)$ will stand for the Jacobian of $\mathrm{F}$ at $z$ in $\mathbb{R}^{n-1}$, and similarly for semi-flows on Banach spaces with the notation $D \mathcal{T}_{t, \eta, \tau}(\psi, y)$ where $(\psi, y)$ lies in a specific product of Banach spaces of continuous functions.

The classical Landau notation $o(\|y\|)$ ("little o") will stand for a term $z$ in $\mathbb{R}^{n-1}$ whose norm $\|z\|$ can be 
neglected with respect to $\|y\|$ in a neighborhood of a point (often 0 ) in $\mathbb{R}^{n-1}$. This notation will be also used for Banach spaces: for instance $o\left(\|\mathrm{~h}\|_{\alpha}\right)$, where $\mathrm{h}$ is in $\mathscr{C}_{\alpha}\left(\mathbb{R}_{-}, \mathbb{R}\right)$.

2.2. Statement of the main result and sketch of the proof. The main result of this paper unveils a frequency-locking for trajectories of the infinite-dimensional dynamical system (1) starting (in some sense) close to the original periodic orbit of $\mathrm{f}$ in $\mathbb{R}^{n}$. This means all trajectories starting in a neighborhood of this orbit in $\mathscr{C}\left([-\tau, 0], \mathbb{R}^{n}\right)$ will be proven to asymptotically converge to a periodic orbit, such periodic orbits being in finite number (depending on $\eta, \tau$ and g). Let's introduce the following notation:

Notation 2.1. Consider the periodic orbit $\Gamma \subset \mathbb{R}^{n}$ of the vector field $\mathrm{f}$. For any $\delta>0$, we denote by $\mathcal{V}_{\Gamma, \tau}(\delta)$ the neighborhood of $\Gamma$ in $\mathscr{C}\left([-\tau, 0], \mathbb{R}^{n}\right)$ of size $\delta$, that is to say the following open set:

$$
\mathcal{V}_{\Gamma, \tau}(\delta)=\left\{\gamma_{0} \in \mathscr{C}\left([-\tau, 0], \mathbb{R}^{n}\right): \sup _{s \in[-\tau, 0]} \inf _{y \in \Gamma}\left\|\gamma_{0}(s)-y\right\|<\delta\right\} .
$$

The frequency-locking we are looking for will be obtained by using normal coordinates. To introduce these coordinates, we first establish the following result (see the proof in Section 3):

Lemma 2.2. There exist $\delta_{0}, \eta_{0}>0$, an open set $\mathcal{V}_{\delta_{0}}$ of $\mathbb{R}^{n}$ containing $\Gamma$ and a $\mathscr{C}^{2}$-diffeomorphism $\Phi$ :

$$
\begin{gathered}
\Phi: \mathcal{V}_{\delta_{0}} \subset \mathbb{R}^{n} \rightarrow \mathbb{S}^{1} \times \mathcal{W}_{\delta_{0}} \subset \mathbb{S}^{1} \times \mathbb{R}^{n-1} \\
x \quad\left(\Phi_{1}(x), \Phi_{2}(x)\right)=(\psi, y)
\end{gathered}
$$

where $\mathcal{W}_{\delta_{0}}=\left\{y \in \mathbb{R}^{n-1}:\|y\| \leq \delta_{0}\right\}$ such that for any $0<\eta<\eta_{0}$, and any $\tau>0$, the diffeomorphism $\Phi$ transforms the solutions $(x(t))_{t \geq 0}$ of (1) starting at initial conditions in the neighborhood $\mathcal{V}_{\Gamma, \tau}\left(\delta_{0}\right)$ of $\Gamma$ in $\mathscr{C}\left([-\tau, 0], \mathbb{R}^{n}\right)$ into the curves $(\bar{\psi}(t), y(t))_{t \geq 0}$ solutions of the system:

$$
\left\{\begin{array}{l}
\frac{d \psi}{d t}=\frac{2 \pi}{T}+\eta \mathrm{g}_{1}(\psi(t), y(t), \psi(t-\tau), y(t-\tau)) \\
\left.\frac{d y}{d t}=A y+\mathrm{h}_{2}(\psi, y)+\eta \mathrm{g}_{2}(\psi(t), y(t)), \psi(t-\tau), y(t-\tau)\right)
\end{array},\right.
$$

where $\psi$ has values in $\mathbb{R}, y$ has values in $\mathbb{R}^{n-1}$, the $\mathscr{C}^{2}$ maps $\mathrm{g}_{1}, \mathrm{~g}_{2}, \mathrm{~h}_{2}$ are $2 \pi$-periodic in the variable $\psi$, and there exists a constant $\mathcal{K}\left(\mathrm{h}_{2}\right)>0$ such that for any $\psi \in \mathbb{R}$ and any $y \in \mathcal{W}_{\delta_{0}}$ we have: $\left\|\mathrm{h}_{2}(\psi, y)\right\| \leq \mathcal{K}\left(\mathrm{h}_{2}\right)\|y\|^{2}$.

The proof of this lemma requires to perform a coordinates transformation for Equation (1), similar to the classical case of a hyperbolic stable periodic orbit for a smooth vector field in $\mathbb{R}^{n}$ (see the book of Hale [17] or [35]). Compared to other works, this coordinate transformation for Equation (1) is not done in the phase space (as in [16]), which is infinite dimensional, but at any time $t$, using the fact that the term $x(t)$ solution of (1) always belongs to $\mathbb{R}^{n}$. In this way we can "formally" use the $\mathscr{C}^{2}$-diffeomorphism $\Phi$ giving the normal coordinates in the non delay situation and obtain a new form of our delay equation (1), more suitable for the obtention of the frequency-locking we are looking for.

Remark 2.3. This means that the $\mathscr{C}^{2}$-diffeomorphism $\Phi$ of Lemma 2.2 gives a bijective correspondance between solutions $(x(t))_{t \geq 0}$ of Equation (1) starting at initial conditions $\gamma_{0}$ close to $\Gamma$ in $\mathscr{C}\left([-\tau, 0], \mathbb{R}^{n}\right)$ and solutions $(\psi(t), y(t))_{t \geq 0}$ of Equation (2) starting at initial conditions $\Phi \circ \gamma_{0}$.

By rescaling time from $t$ to $t^{\prime}=t / \tau$ we are thus led to the following equivalent equation:

$$
\left\{\begin{array}{l}
\frac{d \psi}{d t}=\frac{2 \pi \tau}{T}+\eta \tau \mathrm{g}_{1}(\psi(t), y(t), \psi(t-1), y(t-1)) \\
\left.\frac{d y}{d t}=\tau A y+\tau \mathrm{h}_{2}(\psi, y)+\eta \tau \mathrm{g}_{2}(\psi(t), y(t)), \psi(t-1), y(t-1)\right)
\end{array}\right.
$$

for which the initial conditions are taken in a neighborhood $\mathcal{V}_{\Gamma, 1}(\delta)$ close enough to $\Gamma$ in $\mathscr{C}\left([-1,0], \mathbb{R}^{n}\right)$.

Now, for Equation (3), we observe in Section 3 that after a finite time the value of $\|y(t)\|$ is of order of magnitude $\eta$. Thus, replacing in the equation of the variable $\psi$, we get a delay equation for $\psi$ that roughly speaking does not depend on $y$.

To prove this assertion rigorously, we use an invariant manifold approach similar to the construction of invariant manifolds for flows in $\mathbb{R}^{n}$ : we perform a method inspired by the Perron-Lyapunov method $[9,29,30]$, which comes to applying the Banach Contraction Mapping principle for an integral operator 
coming from the variation of constants formula (see [35]). Such a method require to work on curves defined for infinite backward time, but in general solutions of delay differential equations are not unique in the past: to overcome this problem, we consider the semi-flow of (3) for initial conditions directly defined on the entire set $\mathbb{R}_{-}$, and not on the finite domain $[-1,0]$. A natural choice is the Banach space $\mathscr{C}_{\alpha}\left(\mathbb{R}_{-}, \mathbb{R}\right)$ of functions of growth exponentially bounded on $\mathbb{R}_{-}$for the variable $\psi$, and the Banach space $\mathcal{B}_{\delta_{0}}\left(\mathbb{R}_{-}, \mathbb{R}^{n-1}\right.$ ) of uniformly bounded functions on $\mathbb{R}_{-}$for the variable $y$. On $\mathscr{C}_{\alpha}\left(\mathbb{R}_{-}, \mathbb{R}\right) \times \mathcal{B}_{\delta_{0}}\left(\mathbb{R}_{-}, \mathbb{R}^{n-1}\right)$, the semi-flow of Equation (3) is $\mathscr{C}^{2}$, and we prove in Section 4 (Lemma 4.3) it admits the following $\mathscr{C}^{2}$ invariant manifold in that space:

$$
\mathcal{M}_{\alpha}=\left\{\left(\phi, \mathrm{G}_{\alpha}(\phi)\right), \phi \in \mathscr{C}_{\alpha}\left(\mathbb{R}_{-}, \mathbb{R}\right)\right\},
$$

where $\mathrm{G}_{\alpha}$ is of class $\mathscr{C}^{2}$ with values in $\mathcal{B}_{\delta_{0}}\left(\mathbb{R}_{-}, \mathbb{R}^{n-1}\right)$. A key point in our approach is the existence of an exponent $\boldsymbol{\alpha}_{\mathbf{1}}>0$ (depending on $\eta, \tau$ ) for which this manifold $\mathcal{M}_{\boldsymbol{\alpha}_{1}}$ is in fact globally stable for the semi-flow of (3) (see Theorem 4.5), in the meaning that for any $\left(\phi_{1}, y_{1}\right)$ in $\mathscr{C}([-1,0], \mathbb{R}) \times \mathcal{B}_{\delta_{0} / 2}\left([-1,0], \mathbb{R}^{n-1}\right)$ there exists an initial condition $\left(\phi_{0}, y_{\phi_{0}}\right)$ in $\mathcal{M}_{\boldsymbol{\alpha}_{1}}$ such that we have:

$$
\lim _{t \rightarrow \infty} \sup _{s \in[-1,0]}\left\|\mathcal{T}_{t, \eta, \tau}\left(\phi_{0}, y_{\phi_{0}}\right)(s)-\mathcal{T}_{t+1, \eta, \tau}\left(\phi_{1}, y_{1}\right)(s)\right\|=0
$$

This convergence is not as strong as the foliation result obtained by Chen, Hale, and Tan [7], due to the shift by time $t+1$ in this expression (see Figure 1): however, this global attractivity still permits us to reduce Equation (3) on this manifold $\mathcal{M}_{\boldsymbol{\alpha}_{1}}$. This comes to reducing it to an equation in only one variable, but this time defined by a semi-flow $\mathcal{T}_{\mathcal{M}_{\boldsymbol{\alpha}_{1}}, t, \eta, \tau}$ of class $\mathscr{C}^{2}$ on $\mathscr{C}_{\boldsymbol{\alpha}_{1}}\left(\mathbb{R}_{-}, \mathbb{R}\right)$, and not on $\mathscr{C}([-1,0], \mathbb{R})$. This equation, obtained at the end of Section 4 , has the form:

$$
\frac{d \psi}{d t}=\frac{2 \pi \tau}{T}+\eta \tau \mathcal{G}_{1}\left(\psi_{t}\right)+\eta^{2} \tau \mathcal{H}_{\boldsymbol{\eta}}\left(\psi_{t}\right)
$$

where $\mathcal{H}_{\boldsymbol{\eta}}: \mathscr{C}_{\boldsymbol{\alpha}_{\mathbf{1}}}\left(\mathbb{R}_{-}, \mathbb{R}\right) \rightarrow \mathbb{R}$ is uniformly bounded in $\eta$ (and thus $\eta^{2} \tau \mathcal{H}_{\boldsymbol{\eta}}\left(\psi_{t}\right)$ is a perturbed term of order $\eta$, provided $\eta \tau$ is larger than a constant larger than one). In fact the semi-flow of this equation is not only $\mathscr{C}^{2}$ on $\mathscr{C}_{\alpha_{1}}\left(\mathbb{R}_{-}, \mathbb{R}\right)$ but on $\mathscr{C}_{\alpha}\left(\mathbb{R}_{-}, \mathbb{R}\right)$ for any $\alpha>0$ (only the global stability mentioned above and given by Theorem 4.5 of such a manifold $\mathcal{M}_{\alpha}$ is true for $\left.\alpha=\boldsymbol{\alpha}_{\mathbf{1}}\right)$. The obtention of this equation concludes the first part (composed of Section 3 and Section 4) of this paper.

In the second part (from Section 5) we study this equation in the variable $\psi$, a priori on any space of the form $\mathscr{C}_{\alpha}\left(\mathbb{R}_{-}, \mathbb{R}\right)$, with the purpose of describing its solutions in asymptotic time: in that purpose, we first look at its first order approximation in $\eta$ (Equation (8)). Using averaging techniques we reduce Equation (8) to a much simpler averaged system (15). For this averaged system, we prove the existence of an exponent $\boldsymbol{\alpha}_{\mathbf{2}}>0$ for which we can fully describe the asymptotic behavior of any trajectory starting in $\mathscr{C}_{\boldsymbol{\alpha}_{\mathbf{2}}}\left(\mathbb{R}_{-}, \mathbb{R}\right)$ : this is done in Lemma 6.5 (using Lemma 6.1, Proposition 6.3). The asymptotic behavior, roughly in $\left(\frac{2 \pi \tau}{T}+\omega^{*}\right) t$ where $\omega^{*}$ is a constant depending on $\eta, \tau$ and $g$, is the desired frequency-locking for System (15). It is there that we use the persistence result of normally hyperbolic invariant manifolds [3] of Bates, Lu, Zeng (we recall this theorem in Section 8.1 of the Appendix): it says that if a semi-flow admitting a normally hyperbolic invariant manifold is of class $\mathscr{C}^{1}$ on the Banach space considered, and if its linearized semi-flow restricted to the unstable part associated to this manifold is an isomorphism, then this manifold persists under small perturbations of the semi-flow (see the Appendix).

Applying this result, we conclude there exists an exponent $\boldsymbol{\alpha}_{\mathbf{2}}>0$ such that the asymptotic behavior of any trajectory (starting in $\mathscr{C}_{\boldsymbol{\alpha}_{\mathbf{2}}}\left(\mathbb{R}_{-}, \mathbb{R}\right)$ ) of Equation (8) is given approximately by this term $\left(\frac{2 \pi \tau}{T}+\omega^{*}\right) t$ : this is the statement given by Corollary 6.7, which concludes the second part of this paper.

Finally, in Section 7 we merge the two parts above. The two exponents obtained $\boldsymbol{\alpha}_{\mathbf{1}}>0$ and $\boldsymbol{\alpha}_{\mathbf{2}}>0$ have no reason to be equal but given any initial condition $X_{0} \in \mathscr{C}_{\boldsymbol{\alpha}_{1}}\left(\mathbb{R}_{-}, \mathbb{R}\right)$ we can always modify it on ]$-\infty,-1]$ so that the new initial condition $\tilde{X}_{0}$ belongs to $\mathscr{C}_{\boldsymbol{\alpha}_{1}}\left(\mathbb{R}_{-}, \mathbb{R}\right) \cap \mathscr{C}_{\boldsymbol{\alpha}_{2}}\left(\mathbb{R}_{-}, \mathbb{R}\right)$. Since our goal is to deal with Equation (1) which is defined by a finite time delay (equal to 1 in the rescaled time), we can consider the asymptotic behavior of $\left(\mathcal{T}_{\mathcal{M}_{\alpha_{1}}, t, \eta, \tau}\left(X_{0}\right)(s)\right)_{t \geq 0}$ for time $s \in[-1,0]$, which therefore comes to considering the one of $\left(\mathcal{T}_{\mathcal{M}_{\alpha_{1}}, t, \eta, \tau}\left(\tilde{X_{0}}\right)(s)\right)_{t \geq 0}$. Using this point, we can use Corollary 6.7 of the second part to describe trajectories of the semi-flow $\mathcal{T}_{\mathcal{M}_{\alpha_{1}}}$ : this gives Corollary 7.1. From this, we can conclude that any trajectory of Equation (3) starting with an initial condition in $\mathscr{C}([-1,0], \mathbb{R}) \times \mathcal{B}_{\delta_{0}}\left([-1,0], \mathbb{R}^{n-1}\right)$ converges to a periodic 
orbit (as stated in Theorem 7.3), which periodic orbits are in finite number and defined by the constants $\omega^{*}$ mentioned above. Using the diffeomorphism $\Phi$ of Lemma 2.2, we finally obtain the same conclusion for our original Equation (1). This conclusion (see the end of Section 7) constitutes Theorem A, which is the main result of this paper, and its statement is the following:

Theorem A. There exists $\delta_{0}>0$ for which, given any $L_{1}, L_{2} \geq 1$, there exist $\eta_{0}, M_{0}>0$ such that, for a generic choice of $\eta, \tau$ in the set $\left\{(\eta, \tau) \in \mathbb{R}^{2}: 0<\eta<\eta_{0}, \tau>0, L_{1} \leq \eta \tau \leq L_{2}\right\}$, there exist $p$ real numbers $\omega_{1}^{*}, \cdots, \omega_{p}^{*}$ associated to $p$ functions $\zeta_{1}, \cdots, \zeta_{p} \in \mathscr{C}\left([-\tau, 0], \mathbb{R}^{n}\right)$ satisfying the following:

for any initial condition $\gamma$ in the neighborhood $\mathcal{V}_{\Gamma, \tau}\left(\delta_{0} / 2\right)$ of $\Gamma$ in $\mathscr{C}\left([-\tau, 0], \mathbb{R}^{n}\right)$, there exists an integer $1 \leq i \leq p$ for which the trajectory $\left(\mathcal{T}_{t, \eta, \tau}(\gamma)\right)_{t \geq 0}$ solution of Equation (1) converges asymptotically to the $\operatorname{orbit}\left(\mathcal{T}_{t, \eta, \tau}\left(\zeta_{i}\right)\right)_{t \geq 0}$. Moreover, $\left(\mathcal{T}_{t, \eta, \tau}\left(\zeta_{i}\right)\right)_{t \geq 0}$ is periodic, and of period $A_{i}$ satisfying $\left|A_{i}-\frac{2 \pi}{\frac{2 \pi}{T}+\frac{\omega_{i}^{*}}{\tau}}\right| \leq M_{0} \eta^{2}$.

Interpretation of Theorem A. When $\eta$ is very small and $\tau>0$ very large such that $\eta \tau$ be bounded but non small, there are clusters of synchronization in frequency, through which all trajectories of Equation (1) converge. The number of these clusters is finite and depends on $\eta, \tau$. Each of them is defined by a frequency equal (at first order in $\eta$ ) to $\frac{2 \pi}{T}+\frac{\omega_{i}^{*}}{\tau}$.

Remark 2.4. (i) In this statement, the real numbers $L_{1}, L_{2} \geq 1$ are just used to ensure $\eta \tau$ is bounded and larger than one (which imposes $\tau$ to tend to infinity when $\eta$ tends to 0): for simplicity, one can essentially consider in the first instance that $L_{1}=L_{2}=1$. Since in this case the delay $\tau$ is forced to be equal to $\frac{1}{\eta}$ (which is too restrictive), we take $L_{1}, L_{2} \geq 1$ arbitrarily to avoid this unnecessary restriction on the form of the delay.

(ii) The case where $\tau$ is kept at a fixed value $\tau_{0}$ when $\eta$ varies (or the case where $\tau$ is of order $\eta$ ), is trivial: indeed, in this case, a direct application of the result of Bates, Lu, Zeng ensures that for any $\eta$ small enough, the original periodic orbit $\Gamma$ persists as a stable periodic orbit (unique, in a small neighborhood of $\Gamma$ ) in $\mathscr{C}\left(\left[-\tau_{0}, 0\right], \mathbb{R}^{n}\right)$.

(iii) We will see (Section 6) that for any $1 \leq i \leq p$, we have $\omega_{i}^{*}=\eta \tau G\left(\omega_{i}^{*}+\frac{2 \pi \tau}{T}\right)$, where the map $G$ is $2 \pi$-periodic on $\mathbb{R}$ : thus, when $\eta$ tends to 0 , the period $A_{i}$ converges quadratically to the period $T$ of the original orbit $\Gamma$ in $\mathbb{R}^{n}$.

(iv) Observe that for larger and larger values of $\eta \tau$, the number of fixed points of the map $\eta \tau G\left(\cdot+\frac{2 \pi \tau}{T}\right)$ increases: therefore we recover, as in the paper of Perlikowski and Yanchuk [14] (and as in [1] as well), that the number of periodic orbits increases with the delay. Moreover, except for some pathological values of $\eta, \tau$ in $\left\{(\eta, \tau) \in \mathbb{R}^{2}: 0<\eta<\eta_{0}, \tau>0, L_{1} \leq \eta \tau \leq L_{2}\right\}$, we will prove that the stability of these periodic orbits are given by the slopes of the map $\eta \tau G\left(\cdot+\frac{2 \pi \tau}{T}\right)$ at the fixed points $\omega_{i}^{*}$ : namely, if $\mathrm{F}^{\prime}\left(\omega_{i}^{*}\right)>1$, then the associated periodic orbit is unstable and if $\mathrm{F}^{\prime}\left(\omega_{i}^{*}\right)<1$ and $\mathrm{F}^{\prime}\left(\omega_{i}^{*}\right) \neq 0$, it is stable. Thus, again as in [14] and [1], we recover the fact that roughly speaking, half of the periodic orbits are stable, and the other half unstable (see Figure 2 in Section 6).

The remaining part of this paper is dedicated to the proof of Theorem A, and contains the steps discussed above with all the technical proofs in detail.

\section{Normal coordinates for Equation (1)}

In this section we prove Lemma 2.2 stated in Section 2:

Proof of Lemma 2.2 As the vector field $\mathrm{f}$ is $\mathscr{C}^{3}$ in $\mathbb{R}^{n}$, then as explained in [17] there exists $\delta_{1}>0$, an open set $\mathcal{V}_{\delta_{1}}$ of $\mathbb{R}^{n}$ containing $\Gamma$, and a $\mathscr{C}^{2}$ map $\Psi$ :

$$
\begin{gathered}
\Psi: \mathbb{R} \times \mathcal{W}_{\delta_{1}} \subset \mathbb{R} \times \mathbb{R}^{n-1} \rightarrow \mathcal{V}_{\delta_{1}} \subset \mathbb{R}^{n} \\
(\psi, y) \stackrel{x}{ }
\end{gathered}
$$

where $\mathcal{W}_{\delta_{1}}=\left\{y \in \mathbb{R}^{n-1}:\|y\| \leq \delta_{1}\right\}$, satisfying the relation $\Psi(\psi+2 \pi, y)=\Psi(\psi, y)$ and which induces a diffeomorphism when restricted to $\left[0,2 \pi\left[\times \mathcal{W}_{\delta_{1}}\right.\right.$ such that this diffeomorphism $\Psi$ transforms the solutions of 
the equation $\dot{x}=\mathrm{f}(x)$ into the solutions of the following system:

$$
\left\{\begin{array}{l}
\dot{\psi}=\frac{2 \pi}{T} \\
\dot{y}=A y+\mathrm{h}_{2}(\psi, y)
\end{array}\right.
$$

in which $\psi$ takes values in $\mathbb{R}$, the matrix $A$ of size $n-1$ have eigenvalues $\lambda_{1}, \cdots, \lambda_{n-1}$ all strictly negative, and there exists a constant $\mathcal{K}\left(\mathrm{h}_{2}\right)$ satisfying, for any $\psi \in \mathbb{R}$ and $y \in \mathcal{W}_{\delta_{1}}:\left\|\mathrm{h}_{2}(\psi, y)\right\| \leq \mathcal{K}\left(\mathrm{h}_{2}\right)\|y\|^{2}$. Moreover, in this differential system the map $\mathrm{h}_{2}$ is of class $\mathscr{C}^{2}$ in both variables and satisfies the relation $\mathrm{h}_{2}(\psi+2 \pi, y)=\mathrm{h}_{2}(\psi, y)$.

Under this change of coordinates, the new variables $\psi, y$, are called the normal coordinates (see [35]). Let's denote by $\Phi$ the inverse of the restriction of the map $\Psi$ to $\left[0,2 \pi\left[\times \mathcal{W}_{\delta_{1}}\right.\right.$.

Then, given $\eta>0, \tau>0$ there exists a finite time $t_{0}>0$ such that the curves $(\Phi(x(t)))_{t \in\left[0, t_{0}\right]}=$ $(\psi(t), y(t))_{t \in\left[0, t_{0}\right]}$ (where $x(t)$ denotes a solution of $\dot{x}=\mathrm{f}(x)+\eta \mathrm{g}(x(t), x(t-\tau)$ ) having an initial condition $\gamma$ in the neighborhood $\mathcal{V}_{\Gamma, \tau}\left(\delta_{1}\right)$ of $\Gamma$ in $\left.\mathscr{C}\left([-\tau, 0], \mathbb{R}^{n}\right)\right)$ satisfy:

$$
\forall t \in\left[0, t_{0}\right], y(t) \in \mathcal{W}_{\delta_{1}}
$$

and therefore satisfy, formally:

$$
\left\{\begin{array}{l}
\frac{d \psi}{d t}=\frac{2 \pi}{T}+\eta \frac{\partial \Phi_{1}}{\partial x}(x(t)) \mathrm{g}(\Phi(\psi(t), y(t)), \Phi(\psi(t-\tau), y(t-\tau))) \\
\frac{d y}{d t}=A y+\mathrm{h}_{2}(\psi, y)+\eta \frac{\partial \Phi_{2}}{\partial x}(x(t)) \mathrm{g}(\Phi(\psi(t), y(t)), \Phi(\psi(t-\tau), y(t-\tau)))
\end{array} .\right.
$$
i.e:

In other words, we get that the curves $\left(\Phi_{1}(x(t)), \Phi_{2}(x(t))\right)_{t \in\left[0, t_{0}\right]}=(\psi(t), y(t))_{t \in\left[0, t_{0}\right]}$ satisfy System $(2)$,

$$
\left\{\begin{array}{l}
\frac{d \psi}{d t}=\frac{2 \pi}{T}+\eta \mathrm{g}_{1}(\psi(t), y(t), \psi(t-\tau), y(t-\tau)) \\
\frac{d y}{d t}=A y+\mathrm{h}_{2}(\psi, y)+\eta \mathrm{g}_{2}(\psi(t), y(t), \psi(t-\tau), y(t-\tau))
\end{array}\right.
$$

where $\mathrm{g}_{1}, \mathrm{~g}_{2}$ are functions of class $\mathscr{C}^{2}$ from $\left(\mathbb{R} \times \mathbb{R}^{n-1}\right)^{2}$ to $\mathbb{R}$ which are $2 \pi$-periodic in the variable $\psi$.

Now, in accordance with the statement we are proving, we need to justify this correspondance between System (1) and System (2) is valid for any time $t \geq 0$, not only for time $t \in\left[0, t_{0}\right]$ (i.e that the curves $(\Phi(x(t)))_{t \in\left[0, t_{0}\right]}=(\psi(t), y(t))_{t \in\left[0, t_{0}\right]}$ satisfy System $(2)$ above for any time $t \geq 0$ and not only in $\left.\left[0, t_{0}\right]\right)$. To do this, we first notice the existence of a constant $\sigma>0$ and of a matrix $P \in G L_{n-1}(\mathbb{R})$ (depending only on the matrix $A$, and not on $\mathrm{g}, \eta$, or $\tau$ ) such that:

$$
\forall z \in \mathbb{R}^{n-1}:\left\langle z, P^{-1} A P z\right\rangle \leq-\sigma\|z\|^{2},
$$

and this since all eigenvalues of $A$ are negative: this classic inequality can be seen by applying the Jordan normal form to $A$ (see Lemma 8.1 in the Appendix). We now choose $0<\delta_{0}<\delta_{1}$ satisfying the condition:

$$
-\sigma+\left\|P^{-1}\right\|^{2} \cdot\|P\|^{2} \mathcal{K}\left(\mathrm{h}_{2}\right) \delta_{0}<0
$$

and we choose $C_{0}>0$ large enough so that the inequality

$$
\left[-\sigma+\left\|P^{-1}\right\|^{2} \cdot\|P\|^{2} \mathcal{K}\left(\mathrm{h}_{2}\right) \delta_{0}\right]+\frac{\left\|P^{-1}\right\| \mathcal{K}\left(\mathrm{g}_{2}, \delta_{0}\right)}{C_{0}}<0
$$

be satisfied (where $\left.\mathcal{K}\left(\mathrm{g}_{2}, \delta_{0}\right)=\max _{\psi, \psi^{\prime} \in[0,2 \pi]^{2}, y, y^{\prime} \in \mathcal{W}_{\delta_{0}}^{2}}\left\|\mathrm{~g}_{2}\left[\psi, y, \psi^{\prime}, y^{\prime}\right]\right\|\right)$. Moreover, we reduce the value of $\eta>0$ so that we have $\eta C_{0}\|P\|<\delta_{0}$. Then, given a fixed $\tau>0$, let's consider a trajectory $(\Phi(x(t)))_{t \geq 0}=$ $(\psi(t), y(t))_{t \geq 0}$ starting at an initial condition in the open set $\mathcal{V}_{\Gamma, \tau}\left(\delta_{0}\right)$. Assume, that for a given time $t>0$ 
we have: $\left\|P^{-1} y(t)\right\| \geq \eta C_{0}$ and $\|y(t)\| \leq \delta_{0}$. Then it comes, by Lemma 8.1 of the Appendix:

$$
\begin{aligned}
\frac{1}{2} \frac{d}{d t}\left\|P^{-1} y(t)\right\|^{2} & =\left\langle P^{-1} y(t), P^{-1} \dot{y}(t)\right\rangle \\
& \leq-\sigma\left\|P^{-1} y(t)\right\|^{2}+\left\|P^{-1} y(t)\right\|^{3} \cdot\left\|P^{-1}\right\| \cdot\|P\|^{2} \mathcal{K}\left(\mathrm{h}_{2}\right)+\left\|P^{-1} y(t)\right\|^{2}\left\|P^{-1}\right\| \frac{\mathcal{K}\left(\mathrm{g}_{2}, \delta_{0}\right)}{C_{0}} \\
& \leq\left\|P^{-1} y(t)\right\|^{2} \cdot\left[-\sigma+\left\|P^{-1}\right\|^{2} \cdot\|P\|^{2} \mathcal{K}\left(\mathrm{h}_{2}\right) \delta_{0}+\frac{\left\|P^{-1}\right\| \mathcal{K}\left(\mathrm{g}_{2}, \delta_{0}\right)}{C_{0}}\right] \\
& \leq \eta^{2} C_{0}^{2}\left[-\sigma+\left\|P^{-1}\right\|^{2} \cdot\|P\|^{2} \mathcal{K}\left(\mathrm{h}_{2}\right) \delta_{0}+\frac{\left\|P^{-1}\right\| \mathcal{K}\left(\mathrm{g}_{2}, \delta_{0}\right)}{C_{0}}\right] \\
& <0 .
\end{aligned}
$$

This means the function $t \mapsto\left\|P^{-1} y(t)\right\|$ is locally decreasing at any time $t>0$ for which $\left\|P^{-1} y(t)\right\| \geq \eta C_{0}$ and $\|y(t)\| \leq \delta_{0}$. So for any time $t>0$ large enough, we have:

$$
\left\|P^{-1} y(t)\right\| \leq \eta C_{0}
$$

which implies:

$$
\|y(t)\| \leq \eta C_{0}\|P\|
$$

Setting $\eta_{0}=\frac{\delta_{0}}{C_{0}\|P\|}$, we have proved that for any $0<\eta<\eta_{0}$ and any $\tau>0$, each curve $(\Phi(x(t)))_{t \geq 0}=$ $(\psi(t), y(t))_{t \geq 0}$, (where $x(t)$ is a solution of $\dot{x}=\mathrm{f}(x(t))+\eta \mathrm{g}[x, x(t-\tau)]$ starting at an initial condition in the open set $\left.\mathcal{V}_{\Gamma, \tau}\left(\delta_{0}\right)\right)$ satisfies:

$$
\forall t \geq 0, y(t) \in \mathcal{W}_{\delta_{0}}
$$

and thus the curves $(\Phi(x(t)))_{t \geq 0}=(\psi(t), y(t))_{t \geq 0}$ satisfy Equation (2) for any time $t \geq 0$. QED.

In fact this proof contains two important informations:

Remark 3.1. $\quad$ (i) According to the proof of Lemma 2.2, the trajectories of System (2) starting in a neighborhood of size $\delta_{0}$ of $\Gamma$, contract to a neighborhood of size of magnitude $\eta$ after a finite time.

(ii) With the same $\eta_{0}, \delta_{0}>0$ taken in this proof, we have that if $0<\eta<\frac{\eta_{0}}{2}$, any trajectory starting at an initial condition in a neighborhood of size $\delta_{0} / 2$ of $\Gamma$ remains at any time in this neighborhood.

\section{Construction of a globally stable invariant manifold For (3)}

Since after a finite time the value of $\|y(t)\|$ in Equation (3) is of order of magnitude $\eta$, thus, replacing in the equation of the variable $\psi$, we get a delay equation for $\psi$ that roughly speaking does not depend on $y$ at first order (provided $\eta \tau$ is larger than 1 ): namely an equation of the form $\frac{d \psi}{d t}=\frac{2 \pi \tau}{T}+\eta \tau \mathrm{g}_{0}(\psi(t), 0, \psi(t-1), 0)$. As said in Section 2, to formalize this idea we prove here the existence of a globally stable invariant manifold $\mathcal{M}_{\alpha_{1}}$ for the semi-flow of Equation (3) (see Lemma (4.3) and Theorem 4.5 below) in which the elements $(\psi, y)$ satisfy that the variable $y$ can be expressed as a function of $\psi$.

In the rest of this section, that is to say for Propositions 4.1, 4.2, Lemma 4.3, and in the proof of Theorem 4.5 below, we fix (see Lemma 8.1 of the Appendix) a constant $C>0$ and $\sigma<0$ such that the following holds:

$$
\forall t \geq 0:\left\|e^{A t}\right\| \leq C e^{\sigma t},
$$

where $A$ is the matrix of System (2) and System (3). We also fix $\eta_{0}>0$ and $\delta_{0}>0$ satisfying Lemma 2.2 , and (as in the proof of this lemma), we reduce their value if necessary so that for any $0<\eta<\eta_{0}$, the 
following holds:

$$
\left\{\begin{array}{l}
C \cdot \frac{\mathcal{K}\left(\mathrm{h}_{2}\right) \delta_{0}^{2}+\eta \mathcal{K}\left(\mathrm{g}_{2}, \delta_{0}\right)}{-\sigma} \leq \frac{\delta_{0}}{4} \\
2 C \cdot \frac{\mathcal{K}\left(D \mathrm{~h}_{2}\right) \delta_{0}+\eta \mathcal{K}\left(D \mathrm{~g}_{2}, \delta_{0}\right)}{-\sigma}<1
\end{array}\right.
$$

where $\mathcal{K}\left(D \mathrm{~h}_{2}\right)=\sup _{z \in \mathcal{W}_{\delta_{0}}}\left\|D \mathrm{~h}_{2}(z)\right\|$ and $\mathcal{K}\left(D \mathrm{~g}_{2}, \delta_{0}\right)=\sup _{\mathfrak{z} \in\left([0,2 \pi] \times \mathcal{W}_{\delta_{0}}\right)^{2}}\left\|D \mathrm{~g}_{2}(\mathfrak{z})\right\|$.

The conditions $(\star)$ and $(\star \star)$ are mainly used in the proof of Theorem 4.5 and could have slightly been relaxed for Propositions 4.1, 4.2 and in Lemma 4.3.

Now given $\alpha>0$, and $0<\eta<\eta_{0}, \tau>0$, consider the following map $\mathrm{T}$ defined over the set of curves in $\mathscr{C}_{\alpha}\left(\mathbb{R}_{-}, \mathbb{R}\right) \times \mathcal{B}_{\delta_{0}}\left(\mathbb{R}_{-}, \mathbb{R}^{n-1}\right):$

$$
\begin{aligned}
& \mathrm{T}: \quad(\phi, y) \mapsto \quad \tilde{y}: t \mapsto \int_{-\infty}^{t} e^{\tau A(t-u)}\left[\tau \mathrm{h}_{2}(\phi(u), y(u))+\eta \tau \mathrm{g}_{2}(\phi(u), y(u), \phi(u-1), y(u-1))\right] d u . \\
& \mathscr{C}_{\alpha}\left(\mathbb{R}_{-}, \mathbb{R}\right) \times \mathcal{B}_{\delta_{0}}\left(\mathbb{R}_{-}, \mathbb{R}^{n-1}\right) \rightarrow \mathcal{B}_{\delta_{0}}\left(\mathbb{R}_{-}, \mathbb{R}^{n-1}\right)
\end{aligned}
$$

Proposition 4.1. For any $\alpha>0$, and any $0<\eta<\eta_{0}, \tau>0$, the map $T$ is well defined, that is to say we have:

$$
\forall \phi, y \in \mathscr{C}_{\alpha}\left(\mathbb{R}_{-}, \mathbb{R}\right) \times \mathcal{B}_{\delta_{0}}\left(\mathbb{R}_{-}, \mathbb{R}^{n-1}\right): T(\phi, y) \in \mathcal{B}_{\delta_{0}}\left(\mathbb{R}_{-}, \mathbb{R}^{n-1}\right)
$$

Proof. By choice of $\delta_{0}>0$, for any $(\phi, y)$ in $\mathscr{C}_{\alpha}\left(\mathbb{R}_{-}, \mathbb{R}\right) \times \mathcal{B}_{\delta_{0}}\left(\mathbb{R}_{-}, \mathbb{R}^{n-1}\right)$, for any $\tau>0$ and any $u \leq 0$, we have:

$$
\left\|\left[\tau \mathrm{h}_{2}(\psi(u), y(u))+\eta \tau \mathrm{g}_{2}(\phi(u), y(u), \phi(u-1), y(u-1))\right]\right\| \leq \tau \mathcal{K}\left(\mathrm{h}_{2}\right) \delta_{0}^{2}+\eta \tau \mathcal{K}\left(\mathrm{g}_{2}, \delta_{0}\right)
$$

and thus, by Conditions $(\star)$ and $(\star \star)$ :

$$
\forall t \geq 0,\|\tilde{y}(t)\| \leq \frac{C}{-\sigma}\left(\mathcal{K}\left(\mathrm{h}_{2}\right) \delta_{0}^{2}+\eta \mathcal{K}\left(\mathrm{g}_{2}, \delta_{0}\right)\right)<\delta_{0}
$$

as desired.

The following lemma is the one that will give us the existence of the desired manifold:

Proposition 4.2. For any $\alpha>0$, any $0<\eta<\eta_{0}, \tau>0$, and any $\phi \in \mathscr{C}_{\alpha}\left(\mathbb{R}_{-}, \mathbb{R}\right)$, the map $T$ is a $\frac{1}{2}$-contraction in the second component:

$$
\forall\left(y_{1}, y_{2}\right) \in \mathcal{B}_{\delta_{0}}\left(\mathbb{R}_{-}, \mathbb{R}^{n-1}\right)^{2},\left\|\mathrm{~T}\left(\phi, y_{1}\right)-\mathrm{T}\left(\phi, y_{2}\right)\right\|_{\infty} \leq \frac{1}{2}\left\|y_{1}-y_{2}\right\|_{\infty} .
$$

Proof. Let $\phi \in \mathscr{C}_{\alpha}\left(\mathbb{R}_{-}, \mathbb{R}\right)$ and $\left(y_{1}, y_{2}\right)$ in $\mathcal{B}_{\delta_{0}}\left(\mathbb{R}_{-}, \mathbb{R}^{n-1}\right)$. We have, for any time $t$ in $\mathbb{R}_{-}$:

$$
\begin{aligned}
\left\|\mathrm{T}\left(\phi, y_{1}\right)(t)-\mathrm{T}\left(\phi, y_{2}\right)(t)\right\| & \leq\left(\tau \mathcal{K}\left(D \mathrm{~h}_{2}\right) \delta_{0}+\eta \tau \mathcal{K}\left(D \mathrm{~g}_{2}, \delta\right)\right) \cdot\left\|y_{1}-y_{2}\right\|_{\infty} \int_{-\infty}^{t}\left\|e^{\tau A(t-s)}\right\| d s \\
& \leq \frac{C}{-\sigma}\left(\mathcal{K}\left(D \mathrm{~h}_{2}\right) \delta_{0}+\eta \mathcal{K}\left(D \mathrm{~g}_{2}, \delta_{0}\right)\right) \cdot\left\|y_{1}-y_{2}\right\|_{\infty}
\end{aligned}
$$

and therefore, by $(\star)$ and $(\star \star)$ we have that $\mathrm{T}$ is a $\frac{1}{2}$-contraction in the second component on the Banach space $\mathcal{B}_{\delta_{0}}\left(\mathbb{R}_{-}, \mathbb{R}^{n-1}\right)$ equipped with the norm $\|\cdot\|_{\infty}$.

From Proposition (4.2), we now have:

Lemma 4.3. For any $\alpha>0$ and any $0<\eta<\eta_{0}, \tau>0$, there exists a $\mathscr{C}^{2}$ manifold $\mathcal{M}_{\alpha}$ included in $\mathscr{C}_{\alpha}\left(\mathbb{R}_{-}, \mathbb{R}\right) \times \mathcal{B}_{\delta_{0}}\left(\mathbb{R}_{-}, \mathbb{R}^{n-1}\right)$, invariant for the semi-flow $\mathcal{T}_{t, \eta, \tau}$ associated to (3). This manifold is defined by:

$$
\mathcal{M}_{\alpha}=\left\{\left(\phi, y_{\phi}\right), \phi \in \mathscr{C}_{\alpha}\left(\mathbb{R}_{-}, \mathbb{R}\right)\right\}
$$

where $y_{\phi}$ denotes the unique fixed point of the map $y \mapsto \mathrm{T}(\phi, y)$, with $y \in \mathcal{B}_{\delta_{0}}\left(\mathbb{R}_{-}, \mathbb{R}^{n-1}\right)$. 
Proof. By Proposition 4.2, given a function $\phi \in \mathscr{C}_{\alpha}\left(\mathbb{R}_{-}, \mathbb{R}\right)$ the map $T$ is a contraction in the second component on the Banach space $\left(\mathcal{B}_{\delta_{0}}\left(\mathbb{R}_{-}, \mathbb{R}^{n-1}\right),\|\cdot\|_{\infty}\right)$ : thus by the Contraction Mapping Principle, this map admits a unique fixed point. Denoting by $y_{\phi}$ this unique fixed point, we obtain the desired manifold $\mathcal{M}_{\alpha}$ as a graph of a function in $\phi$.

Now, let us take $\left(\phi_{0}, y_{\phi_{0}}\right) \in \mathcal{M}_{\alpha}$ and a positive time $t>0$, and let us prove that $\mathcal{T}_{t, \eta, \tau}\left(\phi_{0}, y_{\phi_{0}}\right)$ belongs to $\mathcal{M}_{\alpha}$. Recall that by definition of the semi-flow we have:

$$
\begin{aligned}
\mathcal{T}_{t, \eta, \tau}\left(\phi_{0}, y_{\phi_{0}}\right): s \mapsto\left[\psi\left(t+s, \phi_{0}, y_{\phi_{0}}\right), y\left(t+s, \phi_{0}, y_{\phi_{0}}\right)\right] \\
\mathbb{R}_{-} \rightarrow \mathbb{R} \times \mathbb{R}^{n-1},
\end{aligned}
$$

where $\psi\left(t+s, \phi_{0}, y_{\phi_{0}}\right), y\left(t+s, \phi_{0}, y_{\phi_{0}}\right)$ denotes the solution of (3) starting at $\left(\phi_{0}, y_{\phi_{0}}\right)$ and taken at time $t+s$. The first coordinate $\mathcal{T}_{t, \eta, \tau}\left(\phi_{0}, y_{\phi_{0}}\right)_{1}$ is clearly in $\mathscr{C}_{\alpha}\left(\mathbb{R}_{-}, \mathbb{R}\right)$. Indeed, for any $s \in[-t, 0]$, the term $\mathcal{T}_{t, \eta, \tau}\left(\phi_{0}, y_{\phi_{0}}\right)_{1}(s)$ at time $t+s \geq 0$ is equal to:

$$
\frac{2 \pi \tau(t+s)}{T}+\phi_{0}(0)+\eta \tau \int_{0}^{t+s} \mathrm{~g}_{1}\left(\psi\left(u, \phi_{0}, y_{\phi_{0}}\right), y\left(u, \phi_{0}, y_{\phi_{0}}\right), \psi\left(u-1, \phi_{0}, y_{\phi_{0}}\right), y\left(u-1, \phi_{0}, y_{\phi_{0}}\right)\right) d u
$$

in which the integral is bounded (uniformly in $s \in[-t, 0]$ ) by a constant $\mathcal{K}_{t}<+\infty$, which gives:

$$
\left\|\mathcal{T}_{t, \eta, \tau}\left(\phi_{0}, y_{\phi_{0}}\right)_{1}\right\|_{\alpha} \leq \max \left\{\sup _{s \in]-\infty,-t]}\left|\phi_{0}(t+s)\right| e^{\alpha s},\left|\frac{2 \pi \tau t}{T}\right|+\left|\phi_{0}(0)\right|+\eta \tau \mathcal{K}_{t} t\right\}<+\infty .
$$

Now let us consider the second coordinate $\mathcal{T}_{t, \eta, \tau}\left(\phi_{0}, y_{\phi_{0}}\right)_{2}$.

If $s \in \mathbb{R}_{-}$satisfies $t+s \leq 0$, then we have:

$$
\begin{aligned}
& \mathcal{T}_{t, \eta, \tau}\left(\phi_{0}, y_{\phi_{0}}\right)_{2}(s)=y_{\phi_{0}}(t+s) \\
& =\int_{-\infty}^{t+s} e^{\tau A(t+s-u)}\left[\tau \mathrm{h}_{2}\left(\phi_{0}(u), y_{\phi_{0}}(u)\right)+\eta \tau \mathrm{g}_{2}\left(\phi_{0}(u), y_{\phi_{0}}(u), \phi_{0}(u-1), y_{\phi_{0}}(u-1)\right)\right] d u \\
& =\int_{-\infty}^{s} e^{\tau A(s-u)}\left[\tau \mathrm{h}_{2}\left(\phi_{0}(t+u), y_{\phi_{0}}(t+u)\right)\right. \\
& \left.+\eta \tau \mathrm{g}_{2}\left(\phi_{0}(t+u), y_{\phi_{0}}(t+u), \phi_{0}(t+u-1), y_{\phi_{0}}(t+u-1)\right)\right] d u,
\end{aligned}
$$

from which we get the relation: $y_{\phi_{0}}(t+s)=y_{\psi\left(t+\cdot, \phi_{0}, y_{\phi_{0}}\right)}(s)$, and therefore:

$$
\forall s \in]-\infty,-t], \mathcal{T}_{t, \eta, \tau}\left(\phi_{0}, y_{\phi_{0}}\right)_{2}(s)=y_{\mathcal{T}_{t, \eta, \tau}\left(\phi_{0}, y_{\phi_{0}}\right)_{1}}(s) .
$$

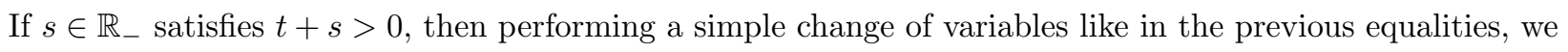
have:

$$
\begin{aligned}
\mathcal{T}_{t, \eta, \tau}\left(\phi_{0}, y_{\phi_{0}}\right)_{2}(s)= & y\left(t+\cdot, \phi_{0}, y_{\phi_{0}}\right)(s) \\
= & \int_{-\infty}^{s} e^{\tau A(s-u)}\left[\tau \mathrm{h}_{2}\left(\psi\left(t+u, \phi_{0}, y_{\phi_{0}}\right), y\left(t+u, \phi_{0}, y_{\phi_{0}}\right)\right)\right. \\
& \left.\quad+\eta \tau \mathrm{g}_{2}\left(\psi\left(t+u, \phi_{0}, y_{\phi_{0}}\right), y\left(t+u, \phi_{0}, y_{\phi_{0}}\right), \psi\left(t+u-1, \phi_{0}, y_{\phi_{0}}\right), y\left(t+u-1, \phi_{0}, y_{\phi_{0}}\right)\right)\right] d u
\end{aligned}
$$

and therefore:

$$
\forall s \in[-t, 0], y\left(t+\cdot, \phi_{0}, y_{\phi_{0}}\right)(s)=\mathrm{T}\left(\psi\left(t+\cdot, \phi_{0}, y_{\phi_{0}}\right), y\left(t+\cdot, \phi_{0}, y_{\phi_{0}}\right)\right)(s),
$$

from which comes:

$$
y\left(t+\cdot, \phi_{0}, y_{\phi_{0}}\right)(s)=y_{\psi\left(t+\cdot, \phi_{0}, y_{\phi_{0}}\right)}(s)
$$

and thus:

$$
\forall s \in[-t, 0], \mathcal{T}_{t, \eta, \tau}\left(\phi_{0}, y_{\phi_{0}}\right)_{2}(s)=y_{\mathcal{T}_{t, \eta, \tau}\left(\phi_{0}, y_{\phi_{0}}\right)_{1}}(s)
$$


Lastly, we remark that $\mathcal{T}_{t, \eta, \tau}\left(\phi_{0}, y_{\phi_{0}}\right)_{2} \in \mathcal{B}_{\delta_{0}}\left(\mathbb{R}_{-}, \mathbb{R}^{n-1}\right)$ since:

$$
\begin{aligned}
\left\|\mathcal{T}_{t, \eta, \tau}\left(\phi_{0}, y_{\phi_{0}}\right)_{2}\right\|_{\infty} & =\max \left\{\sup _{s \in]-\infty,-t]}\left\|y_{\phi_{0}}(t+s)\right\|, \sup _{s \in[-t, 0]}\left\|y_{\mathcal{T}_{t, \eta, \tau}\left(\phi_{0}, y_{\phi_{0}}\right)_{1}}(s)\right\|\right\} \\
& \leq \delta_{0} .
\end{aligned}
$$

We have proved that $\mathcal{T}_{t, \eta, \tau}\left(\phi_{0}, y_{\phi_{0}}\right)$ is in $\mathcal{M}_{\alpha}$, as desired.

Notation 4.4. It will be convenient to denote by $\mathrm{G}_{\alpha}: \mathscr{C}_{\alpha}\left(\mathbb{R}_{-}, \mathbb{R}\right) \rightarrow \mathcal{B}_{\delta_{0}}\left(\mathbb{R}_{-}, \mathbb{R}^{n-1}\right)$ the function of class $\mathscr{C}^{2}$ defining the manifold $\mathcal{M}_{\alpha}$, i.e to set $y_{\phi}:=\mathrm{G}_{\alpha}(\phi)$, in which way the manifold $\mathcal{M}_{\alpha}$ can be written as:

$$
\mathcal{M}_{\alpha}=\left\{\left(\phi, \mathrm{G}_{\alpha}(\phi)\right), \phi \in \mathscr{C}_{\alpha}\left(\mathbb{R}_{-}, \mathbb{R}\right)\right\} .
$$

The conclusion of this section is the next result, which shows that (for some values of $\alpha$ ), $\mathcal{M}_{\alpha}$ is attracting all initial conditions in $\mathscr{C}([-1,0], \mathbb{R}) \times \mathcal{B}_{\delta_{0} / 2}\left([-1,0], \mathbb{R}^{n-1}\right)$ for the semi-flow of Equation (3) (see Figure 1). This is a crucial step for the proof of the main result (Theorem A) of this paper:

Theorem 4.5. There exists $\eta_{0}>0, \delta_{0}>0$ for which the following holds.

Given $0<\eta<\eta_{0}$ and $\tau>0$, there exists $\boldsymbol{\alpha}_{\mathbf{1}}>0$ such that for any $\left(\phi_{1}, y_{1}\right)$ taken in $\mathscr{C}([-1,0], \mathbb{R}) \times$ $\mathcal{B}_{\delta_{0} / 2}\left([-1,0], \mathbb{R}^{n-1}\right)$, there exists an initial condition $\left(\phi_{0}, y_{\phi_{0}}\right)$ in $\mathcal{M}_{\boldsymbol{\alpha}_{\mathbf{1}}} \subset \mathscr{C}_{\boldsymbol{\alpha}_{\mathbf{1}}}\left(\mathbb{R}_{-}, \mathbb{R}\right) \times \mathcal{B}_{\delta_{0}}\left(\mathbb{R}_{-}, \mathbb{R}^{n-1}\right)$ such that for the semi-flow $\mathcal{T}_{t, \eta, \tau}$ of (3) we have:

$$
\lim _{t \rightarrow \infty} \sup _{s \in[-1,0]}\left\|\mathcal{T}_{t, \eta, \tau}\left(\phi_{0}, y_{\phi_{0}}\right)(s)-\mathcal{T}_{t+1, \eta, \tau}\left(\phi_{1}, y_{1}\right)(s)\right\|=0 .
$$

Proof. See Subsection 8.3 of the Appendix.

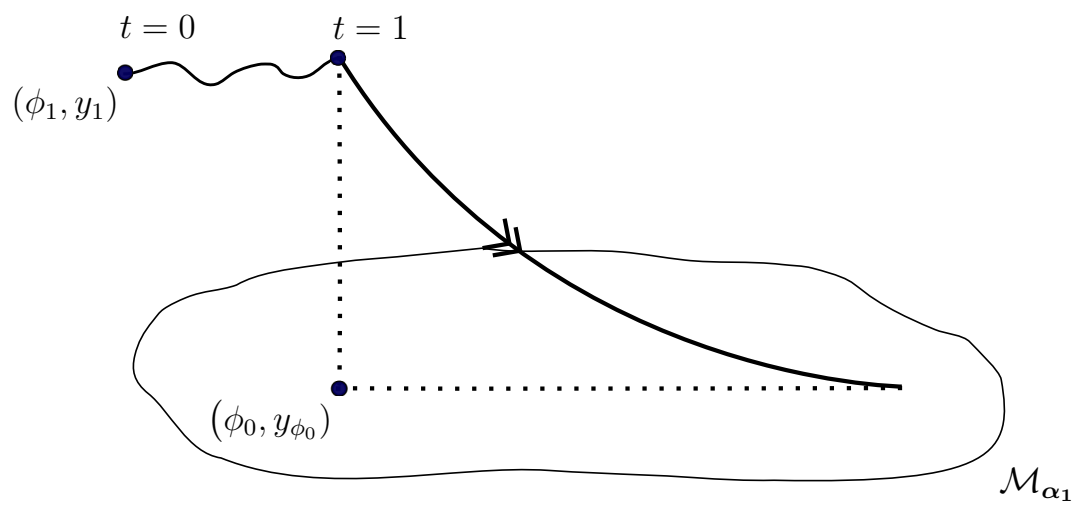

FiguRE 1. Global stability of the manifold $\mathcal{M}_{\boldsymbol{\alpha}_{1}}$ for the semi-flow of Equation (3).

The manifold $\mathcal{M}_{\boldsymbol{\alpha}_{1}}$ included in $\mathscr{C}_{\boldsymbol{\alpha}_{1}}\left(\mathbb{R}_{-}, \mathbb{R}\right) \times \mathcal{B}_{\delta_{0}}\left(\mathbb{R}_{-}, \mathbb{R}^{n-1}\right)$ being attracting, we can therefore restrict our delay differential equation (3) to $\mathcal{M}_{\boldsymbol{\alpha}_{1}}$. Since $\mathcal{M}_{\boldsymbol{\alpha}_{1}}$ is invariant, then given any initial condition $\left(\phi_{0}, y_{\phi_{0}}\right)$ and a positive time $t$, the trajectory $\left(\psi\left(t+s, \phi_{0}, y_{\phi_{0}}\right), y\left(t+s, \phi_{0}, y_{\phi_{0}}\right)\right)_{s \leq 0}$ solution of the delay equation starting at $\left(\phi_{0}, y_{\phi_{0}}\right)$ satisfies the following relation:

$$
\forall s \leq 0, y_{t}\left(\cdot, \phi_{0}, y_{\phi_{0}}\right)(s)=\mathrm{G}_{\boldsymbol{\alpha}_{\mathbf{1}}}\left(\psi_{t}\left(\cdot, \phi_{0}, y_{\phi_{0}}\right)\right)(s) .
$$

Moreover, using the fact that after a finite time $t>0$, the term $y\left(t, \phi_{0}, y_{\phi_{0}}\right.$ ) is of order of magnitude $\eta$ (as a consequence of Lemma 2.2), and using a Taylor expansion we can write:

$$
\begin{aligned}
\dot{\psi}=\frac{2 \pi \tau}{T} & +\eta \tau \mathrm{g}_{1}(\psi(t), 0, \psi(t-1), 0)+\eta^{2} \tau D \mathrm{~g}_{1}(\psi(t), 0, \psi(t-1), 0)\left(0, \frac{y(t)}{\eta}, 0, \frac{y(t-1)}{\eta}\right) \\
& +\epsilon_{1}(y(t), y(t-1)) \cdot\|y(t)\|+\epsilon_{2}(y(t), y(t-1)) \cdot\|y(t-1)\|
\end{aligned}
$$


where the terms $\epsilon_{1,2}(y(t), y(t-1))$ tend to 0 when $\|y(t)\|$ and $\|y(t-1)\|$ tend to 0 . Therefore, Theorem 4.5 tells us that, given any $\left(\phi_{0}, y_{\phi_{0}}\right) \in \mathcal{M}_{\boldsymbol{\alpha}_{1}}$, the trajectory starting at $\left(\phi_{0}, y_{\phi_{0}}\right)$ satisfies the equation in only one variable:

$$
\frac{d \psi}{d t}=\frac{2 \pi \tau}{T}+\eta \tau \mathcal{G}_{\mathbf{1}}\left(\psi_{t}\right)+\eta^{2} \tau \mathcal{H}_{\boldsymbol{\eta}}\left(\psi_{t}\right)
$$

where $\mathcal{G}_{1}: \mathscr{C}_{\boldsymbol{\alpha}_{1}}\left(\mathbb{R}_{-}, \mathbb{R}\right) \rightarrow \mathbb{R}$ is the function of class $\mathscr{C}^{2}$ defined by $\mathcal{G}_{\mathbf{1}}(\phi)=\mathrm{g}_{1}[\phi(0), 0, \phi(-1), 0]$, and $\mathcal{H}_{\eta}: \mathscr{C}_{\boldsymbol{\alpha}_{1}}\left(\mathbb{R}_{-}, \mathbb{R}\right) \rightarrow \mathbb{R}$ is $\mathscr{C}^{2}$ as well (since $\mathrm{G}_{\boldsymbol{\alpha}_{1}}$ is $\mathscr{C}^{2}$ ) and uniformly bounded in $\eta$.

Therefore, the equation truncated at first order in $\eta$ reads (provided $\eta \tau$ is larger than a constant $L \geq 1$ ):

$$
\frac{d \psi}{d t}=\frac{2 \pi \tau}{T}+\eta \tau \mathrm{h}(\psi(t), \psi(t-1))
$$

where $\mathrm{h}: \mathbb{R} \times \mathbb{R} \rightarrow \mathbb{R}$ defined by the relation $\mathrm{h}(\psi(t), \psi(t-1))=\mathrm{g}_{1}[\psi(t), 0, \psi(t-1), 0]$ is of class $\mathscr{C}^{2}$ and is $2 \pi$-periodic in both variables.

Remark 4.6. $\quad$ (i) Notice that, we must consider Equation (6) (and Equation (7)) on Banach spaces defined on $\mathbb{R}_{-}$and not on $[-1,0]$ since it results from the reduction to the manifold $\mathcal{M}_{\boldsymbol{\alpha}_{1}}$.

(ii) Equation (6) is in fact not only a delay equation with a $\mathscr{C}^{2}$ semi-flow on the Banach space $\mathscr{C}_{\boldsymbol{\alpha}_{\mathbf{1}}}\left(\mathbb{R}_{-}, \mathbb{R}\right)$, but on $\mathscr{C}_{\boldsymbol{\alpha}}\left(\mathbb{R}_{-}, \mathbb{R}\right)$ for any $\alpha>0$ : it's the convergence given by Theorem 4.5 which is only valid for some choices of exponents depending on $\eta$ and $\tau$.

\section{Using Averaging theory to study Equation (7)}

Now in Equation (7), the term $\tau / T$ is taken large enough: therefore the phase is rotating very quickly. To take it into account, we set:

$$
\begin{cases}\beta(t) & :=\frac{2 \pi \tau}{T} \cdot t \\ \phi(t) & :=\psi(t)-\beta(t)\end{cases}
$$

and embed the equation (7) in $\mathbb{R}^{2}$, by considering:

$$
\left\{\begin{array}{l}
\dot{\beta}=\frac{2 \pi \tau}{T} \\
\dot{\phi}=\eta \tau \mathrm{h}(\phi(t)+\beta(t), \phi(t-1)+\beta(t-1)) .
\end{array}\right.
$$

We can study Equation (7) through this embedding in $\mathbb{R}^{2}$ : indeed the equation in $\phi$ is the same equation as the one in $\psi$. Now, as $\mathrm{h}$ is of class $\mathscr{C}^{2}, 2 \pi$-periodic, therefore we can express $\mathrm{h}$ in Fourier series, using the basis given by functions $(s, t) \mapsto \exp (i(m s+n t))$, for any integers $m, n$ in $\mathbb{Z}$. Doing this, we get that $\phi$ has an equation of the form:

$$
\frac{d \phi}{d t}=\eta \tau \sum_{(m, n) \in \mathbb{Z} \times \mathbb{Z}} a_{m, n} e^{i\left(m \phi(t)-n \phi(t-1)+2 \pi n \frac{\tau}{T}\right)} e^{2 \pi i \frac{\tau}{T}(m-n) t},
$$

where $\left(a_{m, n}\right)_{(m, n) \in \mathbb{Z} \times \mathbb{Z}}$ is the family of Fourier coefficients of the function $\mathrm{h}$. Therefore, the reformulation of Equation (8) in the infinite dimensional setting takes the form:

(10)

$$
\left\{\begin{array}{l}
\frac{d \beta_{t}}{d t}(0)=\frac{2 \pi \tau}{T} \\
\frac{d \phi_{t}}{d t}(0)=\eta \tau \sum_{m \in \mathbb{Z}} a_{m, m} e^{i m\left(\phi_{t}(0)-\phi_{t}(-1)+2 \pi \frac{\tau}{T}\right)}+\eta \tau \sum_{(m \neq n)} a_{m, n} e^{i\left(m \phi_{t}(0)-n \phi_{t}(-1)+2 \pi n \frac{\tau}{T}\right)} e^{i(m-n) \beta_{t}(0)}
\end{array} .\right.
$$

As $\tau / T$ is taken very large, the second sum in the right hand side of the equation in $\dot{\phi}$ is very small, and can be skipped at first order in $\eta$ : to formalize this point rigorously, we are now going to use in a hidden way averaging theory.

For $t \geq 0$, let us set $\phi_{t}^{\text {new }}:=\phi_{t}-\eta \tau \Re\left(\sum_{(m \neq n) \in \mathbb{Z} \times \mathbb{Z}} a_{m, n} e^{i\left(m \phi_{t}-n \phi_{t-1}+2 \pi n \frac{\tau}{T}\right)} \int_{0}^{t} e^{i(m-n) \beta_{s}} d s\right)$ (which corresponds to defining a Krylov-Bogoliubov-Mitropolsky type of transformation, as in the classical Averaging 
theory). That is to say we set:

$$
\forall s \leq 0, \phi_{t}^{\text {new }}(s):=\phi_{t}(s)-\eta \Re\left(\sum_{(m \neq n) \in \mathbb{Z} \times \mathbb{Z}} \frac{a_{m, n}}{2 i \pi \frac{1}{T}(m-n)} e^{i\left(m \phi_{t}(s)-n \phi_{t-1}(s)+2 \pi n \frac{\tau}{T}\right)}\left(e^{i(m-n) \beta_{t}(s)}-1\right)\right),
$$

in which way the new coordinate $\phi_{t}^{\text {new }}$ is a small perturbation of $\phi_{t}$.

To study Equation (10), we want to make use of $\phi_{t}^{\text {new }}$ as new variables. We thus need to verify that the definition of $\phi_{t}^{\text {new }}$ above can be somehow considered as a change of variables (which is the statement of Proposition 5.2 below). In this purpose we precise the following notation:

Notation 5.1. Given $\alpha>0$, we denote by $\left(\mathscr{C}_{\alpha}\left(\mathbb{R}_{-}, \mathbb{R}\right)^{2},\|(\cdot, \cdot)\|_{\alpha}\right)$ the product Banach space $\mathscr{C}_{\alpha}\left(\mathbb{R}_{-}, \mathbb{R}\right) \times$ $\mathscr{C}_{\alpha}\left(\mathbb{R}_{-}, \mathbb{R}\right)$ equipped with the norm $\|(\cdot, \cdot)\|_{\alpha}$ defined by the relation

$$
\forall(\beta, \phi) \in \mathscr{C}_{\alpha}\left(\mathbb{R}_{-}, \mathbb{R}\right)^{2},\|(\beta, \phi)\|_{\alpha}:=\max \left(\|\beta\|_{\alpha},\|\phi\|_{\alpha}\right) .
$$

Proposition 5.2. Given $\alpha>0$, let's consider the maps $\mathbb{T}_{1}, \mathbb{T}_{2}, \mathbb{T}_{[-1]}$ defined by:

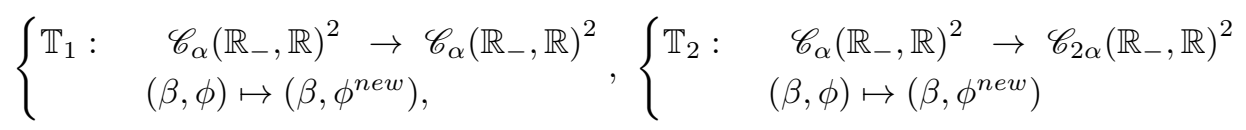

and

$$
\left\{\begin{array}{cc}
\mathbb{T}_{[-1]}: \quad \mathscr{C}_{\alpha}\left(\mathbb{R}_{-}, \mathbb{R}\right)^{2} \rightarrow \mathscr{C}_{2 \alpha}\left(\mathbb{R}_{-}, \mathbb{R}\right)^{2} \\
\left(\beta, \phi^{\text {new }}\right) \mapsto(\beta, \phi)=\mathbb{T}_{1}^{-1}\left(\beta, \phi^{\text {new }}\right)
\end{array}\right.
$$

where the spaces $\mathscr{C}_{\alpha}\left(\mathbb{R}_{-}, \mathbb{R}\right)^{2}$ and $\mathscr{C}_{2 \alpha}\left(\mathbb{R}_{-}, \mathbb{R}\right)^{2}$ are equipped with the norm $\|(\cdot, \cdot)\|_{\alpha}$ and $\|(\cdot, \cdot)\|_{2 \alpha}$. Then, the following holds:

(i) The map $\mathbb{T}_{1}$ is a bi-Lipschitz homeomorphism.

(ii) The map $\mathbb{T}_{2}$ is of class $\mathscr{C}^{1}$.

(iii) The map $\mathbb{T}_{[-1]}$ is of class $\mathscr{C}^{1}$.

Proof. See Appendix, Subsection 8.4.

Remark 5.3. For any given $\alpha>0$, the transformation $(\beta, \phi) \mapsto\left(\beta, \phi^{\text {new }}\right)$ is not a diffeomorphism from $\mathscr{C}_{\alpha}\left(\mathbb{R}_{-}, \mathbb{R}\right)^{2}$ to itself, i.e if we consider the same norm $\left.\|\cdot\|_{\alpha}\right)$. However the map $\mathbb{T}_{[-1]}$ plays the role of the inverse of $\mathbb{T}_{2}$, from which we are now going to prove (in Lemma 5.4 below) that it is sufficient to study Equation (10) through its version with the variable $\phi^{\text {new }}$.

As $\mathrm{h}$ is of class $\mathscr{C}^{2}$, the Fourier series of h converges normally. Therefore we can differentiate under the sign sum in the expression of $\phi_{t}^{\text {new }}$ in $s=0$ to get, for any $t \geq 1$ :

$$
\left\{\begin{aligned}
\frac{d \beta_{t}}{d t}(0)= & \frac{2 \pi \tau}{T} \\
\frac{d \phi_{t}^{\text {new }}}{d t}(0)= & \eta \tau \Re\left(\sum_{m \in \mathbb{Z}} a_{m, m} e^{i m\left(\phi_{t}(0)-\phi_{t}(-1)+2 \pi \frac{\tau}{T}\right)}\right)- \\
& \left.\eta \Re\left(\sum_{(m \neq n)} \frac{a_{m, n}}{2 \frac{\pi}{T}(m-n)} e^{i\left(m \phi_{t}(0)-n \phi_{t}(-1)+2 \pi n \frac{\tau}{T}\right)}\right)\left(m \dot{\phi}_{t}(0)-n \dot{\phi}_{t}(-1)\right)\left(e^{i(m-n) \beta_{t}(0)}-1\right)\right) .
\end{aligned}\right.
$$

It is not restrictive to look at Equation (12) for time $t \geq 1$ since we are interested in asymptotic behavior (i.e for large time $t>0$ ) of trajectories of our initial Equation (1).

Now, by Item (i) of Proposition 5.2, we can replace $\phi(t)$ by $\mathbb{T}_{1}^{-1}\left(\beta_{t}, \phi_{t}^{\text {new }}\right)(0)$, which leads us to the following crucial Lemma:

Lemma 5.4. The variable $\phi^{\text {new }}$ given by the equality (11) satisfies, for any time $t \geq 1$, a delay equation of the form:

$$
\begin{cases}\dot{\beta}(t) & =\frac{2 \pi \tau}{T} \\ \dot{\phi}^{\text {new }}(t) & =\mathcal{G}^{\text {new }}\left[\phi_{t}^{\text {new }}, \beta_{t}\right]\end{cases}
$$


in which $\mathcal{G}^{\text {new }}$ is a functional from $\mathscr{C}_{\alpha}\left(\mathbb{R}_{-}, \mathbb{R}\right)^{2}$ to $\mathbb{R}$, of class $\mathscr{C}^{1}$ for any $\alpha>0$. Moreover, the truncation of the second equation in (13) at first order in $\eta$ reads (provided $\eta \tau$ is larger than a constant $L \geq 1$ ):

$$
\dot{\phi}^{\text {new }}(t)=\eta \tau \Re\left(\sum_{m \in \mathbb{Z}} a_{m, m} e^{i m\left(\phi^{n e w}(t)-\phi^{\text {new }}(t-1)+2 \pi \frac{\tau}{T}\right)}\right) .
$$

Proof. The second equation in (12) is of the form $\dot{\phi}^{\text {new }}(t)=\mathrm{G}_{0}{ }^{\text {new }}[\phi(t), \phi(t-1), \dot{\phi}(t), \dot{\phi}(t-1), \beta(t)]$ with $\mathrm{G}_{0}{ }^{\text {new }}$ defined from $\mathbb{R}^{5}$ to $\mathbb{R}$, and $\mathscr{C}^{1}$ since the Fourier series of h converges normally. Now, for time $t \geq 1$, the two terms $\phi(t), \phi(t-1)$ satisfy Equation (9): thus $\dot{\phi}(t), \dot{\phi}(t-1)$ depend smoothly on $\phi(t), \phi(t-1), \phi(t-$ $2), \beta(t), \beta(t-1)$.

This yields that the second equation in (12) has the form $\dot{\phi}^{\text {new }}(t)=\mathrm{G}_{0}{ }^{\text {new }}[\phi(t), \phi(t-1), \phi(t-2), \beta(t), \beta(t-1)]$ with $\mathrm{G}_{0}{ }^{\text {new }}$ of class $\mathscr{C}^{1}$ from $\mathbb{R}^{5}$ to $\mathbb{R}$, which can be rewritten in a functional way under the form

$$
\dot{\phi}^{\text {new }}(t)=\mathcal{G}_{0}^{\text {new }}\left[\phi_{t}, \beta_{t}\right]
$$

in which $\mathcal{G}_{0}^{\text {new }}: \mathscr{C}_{2 \alpha}\left(\mathbb{R}_{-}, \mathbb{R}\right)^{2} \rightarrow \mathbb{R}$ inherits the smoothness from $\mathrm{G}_{0}{ }^{\text {new }}$ (and this for any fixed $\alpha>0$ ).

Finally by Item (iii) of Proposition 5.2, the map $\mathbb{T}_{[-1]}$ is $\mathscr{C}^{1}$ from $\mathscr{C}_{\alpha}\left(\mathbb{R}_{-}, \mathbb{R}\right)^{2}$ to $\mathscr{C}_{2 \alpha}\left(\mathbb{R}_{-}, \mathbb{R}\right)^{2}$, which means the variable $\phi^{\text {new }}$ given by the equality (11) satisfies, for any time $t \geq 1$, a delay differential equation of the form:

$$
\begin{cases}\dot{\beta}(t) & =\frac{2 \pi \tau}{T} \\ \dot{\phi}^{\text {new }}(t) & =\mathcal{G}^{\text {new }}\left[\phi_{t}^{\text {new }}, \beta_{t}\right]\end{cases}
$$

in which $\mathcal{G}^{\text {new }}$ is a $\mathscr{C}^{1}$ functional from $\mathscr{C}_{\alpha}\left(\mathbb{R}_{-}, \mathbb{R}\right)^{2}$ to $\mathbb{R}$. By Item (i) of Proposition 5.2, the variable $\phi(t)$ is a Lipschitz perturbation (in $\phi^{\text {new }}(t)$ ) of the variable $\phi^{\text {new }}(t)$ : therefore the truncation of this equation at first order in $\eta$ reads as the formula given by (14).

Thus, for our study in asymptotic time of the delay equation (7), we have proved this equation can be seen, for any time $t \geq 1$, as a $\mathscr{C}^{1}$ perturbation of the second equation of the following (averaged) delay differential system:

$$
\begin{cases}\dot{\beta}(t) & =\frac{2 \pi \tau}{T} \\ \dot{\phi}^{\text {new }}(t) & =\eta \tau \mathrm{G}\left[\phi^{\text {new }}(t)-\phi^{\text {new }}(t-1)+\theta_{\tau}\right]\end{cases}
$$

where $\mathrm{G}$ is a $2 \pi$-periodic function of class $\mathscr{C}^{2}$ defined in Fourier Series by the coefficients $\left(a_{m, m}\right)_{m \in \mathbb{Z}}$ in Equation (14) above, and $\theta_{\tau}=2 \pi \frac{\tau}{T}$.

\section{Study of the Dynamics of the AVERAGed EqUation}

In order to analyze System (15), it is useful to introduce the quantity, for time $t \geq 0$ :

$$
\omega(t)=\phi^{\text {new }}(t)-\phi^{\text {new }}(t-1) .
$$

Since the two variables in (15) are separate, we can analyse only the equation in $\phi^{\text {new }}$ in (15). Rewriting it with the new variable $\omega$, we are led to study:

$$
\frac{d \omega}{d t}=\mathrm{F}(\omega(t))-\mathrm{F}(\omega(t-1)), \quad t \geq 0
$$

in which the function $\mathrm{F}$ is defined on $\mathbb{R}$ by the relation $\mathrm{F}(x)=\eta \tau \mathrm{G}\left(x+\theta_{\tau}\right)$. This equation has the remarkable property that any of its trajectories converges to a constant in $\mathbb{R}$, as stated in the next lemma:

Lemma 6.1. Let $\alpha>0$, and $\eta>0, \tau>0$. For any $\omega_{0} \in \mathscr{C}_{\alpha}\left(\mathbb{R}_{-}, \mathbb{R}\right)$, there exists a constant $C_{\omega_{0}}$ such that the solution $\omega\left(\cdot, \omega_{0}\right)$ of the delay equation (16) with initial condition $\omega_{0}$ satisfies: $\omega\left(t, \omega_{0}\right) \rightarrow C_{\omega_{0}}$ when $t$ tends to infinity. 
Proof. 1st Step: Let us define $\varphi(x)=\int_{0}^{x} \mathrm{~F}(s) d s$, for any real $x$. The function $\varphi$ is somehow going to play the role of a Lyapunov function. Consider an initial condition $\omega_{0} \in \mathscr{C}_{\alpha}\left(\mathbb{R}_{-}, \mathbb{R}\right)$, and the solution $\omega:=\omega\left(\cdot, \omega_{0}\right)$ starting at $\omega_{0}$. We are going to prove in this first step that $\left|\int_{0}^{+\infty} \dot{\omega}(s)^{2} d s\right|<+\infty$ using this functional $\varphi$. We have, for any time $t \geq 0$ :

$$
\begin{aligned}
\frac{d}{d t} \varphi(\omega(t)) & =\mathrm{F}(\omega(t)) \dot{\omega}(t) \\
& =\mathrm{F}(\omega(t))[\mathrm{F}(\omega(t))-\mathrm{F}(\omega(t-1))] \\
& =\frac{\mathrm{F}(\omega(t))^{2}}{2}-\frac{\mathrm{F}(\omega(t-1))^{2}}{2}+\frac{1}{2}[\mathrm{~F}(\omega(t))-\mathrm{F}(\omega(t-1))]^{2} \\
& =\frac{\mathrm{F}(\omega(t))^{2}}{2}-\frac{\mathrm{F}(\omega(t-1))^{2}}{2}+\frac{1}{2} \dot{\omega}(t)^{2}
\end{aligned}
$$

and therefore by integrating from 0 to $t$ :

$$
\begin{aligned}
\varphi(\omega(t))-\varphi(\omega(0)) & =\int_{0}^{t} \frac{\mathrm{F}(\omega(s))^{2}}{2} d s-\int_{-1}^{t-1} \frac{\mathrm{F}(\omega(s))^{2}}{2} d s+\frac{1}{2} \int_{0}^{t} \dot{\omega}(s)^{2} d s \\
& =\int_{t-1}^{t} \frac{\mathrm{F}(\omega(s))^{2}}{2} d s-\int_{-1}^{0} \frac{\mathrm{F}(\omega(s))^{2}}{2} d s+\frac{1}{2} \int_{0}^{t} \dot{\omega}(s)^{2} d s .
\end{aligned}
$$

Now, as $\omega(t)$ is bounded (since $\mathrm{F}$ is bounded) we have :

$$
\sup _{t \in \mathbb{R}}|\varphi(\omega(t))-\varphi(\omega(0))|<+\infty .
$$

Moreover, the triangle inequality clearly gives:

$$
\sup _{t \in \mathbb{R}}\left|\varphi(\omega(t))-\varphi(\omega(0))-\int_{t-1}^{t} \frac{\mathrm{F}(\omega(s))^{2}}{2} d s+\int_{-1}^{0} \frac{\mathrm{F}(\omega(s))^{2}}{2} d s\right|<+\infty,
$$

and thus we get:

$$
\left|\int_{0}^{+\infty} \dot{\omega}(s)^{2} d s\right|<+\infty
$$

and this whatever be the initial condition $\omega_{0}$.

2nd Step: Now as $\dot{\omega}(t)$ is bounded and $\ddot{\omega}(t)$ is bounded as well (since $\mathrm{F}^{\prime}$ is bounded), therefore $\dot{\omega}(t)$ must tend to 0. Let's prove this assertion.

Assume the contrary, that is to say assume we have: $\exists \epsilon_{0}>0, \forall A>0, \exists t \geq A, \dot{\omega}(t) \geq \epsilon_{0}$. Thus, fixing such an $\epsilon_{0}>0$, there exists a strictly increasing sequence of positive numbers $\left(a_{n}\right)_{n \geq 1}$ such that:

$$
\forall n \geq 1: \dot{\omega}_{\left[\left[a_{2 n+1}, a_{2 n+2}\right]\right.} \geq \frac{\epsilon_{0}}{2} .
$$

For such a sequence $\left(a_{n}\right)_{n \geq 1}$ we can assume without restricting the generality that exists a constant $\epsilon_{1}>0$ such that for any $n \geq 1, \dot{\omega}\left(a_{2 n+2}\right)-\dot{\omega}\left(a_{2 n+1}\right) \geq \epsilon_{1}$ : otherwise the sequence of general term $\dot{\omega}\left(a_{2 n+2}\right)-$ $\dot{\omega}\left(a_{2 n+1}\right)$ would be approaching 0 and thus $\left(\dot{\omega}\left(a_{n}\right)\right)_{n \geq 1}$ will be asymptotically bounded from below by a positive constant. This is absurd by convergence of the integral of $\dot{\omega}^{2}$.

Now let's apply the mean value equality: $\forall n \geq 1, \dot{\omega}\left(a_{2 n+2}\right)-\dot{\omega}\left(a_{2 n+1}\right)=\ddot{\omega}\left(c_{n}\right)\left(a_{2 n+2}-a_{2 n+1}\right) \geq \epsilon_{1}$, where $c_{n}$ is a real number in $\left[a_{2 n+1}, a_{2 n+2}\right]$. Since $\ddot{\omega}$ is bounded the sequence $\left(a_{2 n+2}-a_{2 n+1}\right)_{n \geq 1}$ is therefore bounded from below by a positive constant. Thus:

$$
\sum_{n=0}^{+\infty} \int_{a_{2 n+1}}^{a_{2 n+2}} \dot{\omega}(s)^{2} d s \geq \sum_{n=0}^{+\infty}\left(a_{2 n+2}-a_{2 n+1}\right) \frac{\epsilon_{0}^{2}}{4}>+\infty,
$$


which is a contradiction. So $\dot{\omega}(t)$ tends to 0 , as desired.

3rd Step: Finally let's prove that $\omega(t)$ tends to a constant depending only on $\mathrm{F}$ when $t$ tends to infinity. Taking again the expression of $\varphi(\omega(t))-\varphi(\omega(0))$ written above, we have, by the mean value equality:

$$
\forall t \geq 0: \varphi(\omega(t))=\varphi(\omega(0))+\frac{\mathrm{F}\left(\omega\left(c_{t}\right)\right)^{2}}{2}-\int_{-1}^{0} \frac{\mathrm{F}(\omega(s))^{2}}{2} d s+\frac{1}{2} \int_{0}^{t} \dot{\omega}(s)^{2} d s,
$$

where $c_{t} \in[t-1, t]$. By the 1st Step we thus get the existence of a constant $c e_{\omega_{0}}$ (depending on $\mathrm{F}$ and on the initial condition $\omega_{0}$ ) such that

$$
\varphi(\omega(t))-\frac{\mathrm{F}\left(\omega\left(c_{t}\right)\right)^{2}}{2} \rightarrow c t \omega_{\omega_{0}}
$$

when $t$ tends to infinity. Then by the mean value equality again,

$$
\varphi(\omega(t))-\frac{\mathrm{F}(\omega(t))^{2}}{2}=\varphi(\omega(t))-\frac{\mathrm{F}\left(\omega\left(c_{t}\right)\right)^{2}}{2}+\mathrm{F}\left(\omega\left(d_{t}\right)\right) \mathrm{F}^{\prime}\left(\omega\left(d_{t}\right)\right) \dot{\omega}\left(d_{t}\right)\left(c_{t}-t\right)
$$

where $d_{t} \in\left[c_{t}, t\right]$. Now as $\mathrm{F}$ and $\mathrm{F}^{\prime}$ are bounded and as $d_{t}$ tends to $+\infty$ with $t$, it comes:

$$
\varphi(\omega(t))-\frac{\mathrm{F}(\omega(t))^{2}}{2} \rightarrow c t e_{\omega_{0}} .
$$

Next, by the mean value equality again, there exists $d_{\omega(t)} \in[\omega(0), \omega(t)]$ such that the following holds:

$$
\varphi(\omega(t))-\frac{\mathrm{F}(\omega(t))^{2}}{2}=\varphi(\omega(0))-\frac{\mathrm{F}(\omega(0))^{2}}{2}+\left[\varphi^{\prime}\left(d_{\omega(t)}\right)-\mathrm{F}\left(d_{\omega(t)}\right) \mathrm{F}^{\prime}\left(d_{\omega(t)}\right)\right] \cdot[\omega(t)-\omega(0)] .
$$

In the last relation, the left part converges, and the term $\left[\varphi^{\prime}\left(d_{\omega(t)}\right)-\mathrm{F}\left(d_{\omega(t)}\right) \mathrm{F}^{\prime}\left(d_{\omega(t)}\right)\right]$ is bounded and does not tend to 0 , since $\mathrm{F}^{\prime}$ does not tend to 1 . Consequently, for any initial condition $\omega_{0} \in \mathscr{C}_{\alpha}\left(\mathbb{R}_{-}, \mathbb{R}\right)$, the term $\omega(t)-\omega(0)$ must tend to a constant depending on $\omega_{0}$ when $t$ tends to infinity.

Remark 6.2. In fact, it is easy to see that Equation (16) admits a Lyapunov function, namely the one defined by the relation:

$$
L_{t}\left(\omega_{0}\right):=\varphi\left(\omega\left(t, \omega_{0}\right)\right)-\frac{1}{2} \int_{t-1}^{t} \mathrm{~F}\left(\omega\left(u, \omega_{0}\right)\right)^{2} d u .
$$

The reader might have noticed that all constant initial conditions on $[-1,0]$ are solutions of (16). Among them, we have:

Proposition 6.3. Let $\eta>0$ and $\tau>0$. Consider $\mathrm{c} \in \mathbb{R}$ such that $\mathrm{F}^{\prime}(\mathrm{c}) \neq 0$, and the characteristic equation $-\lambda+\mathrm{F}^{\prime}(\omega)\left[1-e^{-\lambda}\right]=0$ (where $\lambda \in \mathbb{C}$ ), associated to the linearized equation of (16). Then:

(i) There exists only one multiple eigenvalue which is 0 , and it is a double eigenvalue, occuring when $\mathrm{F}^{\prime}(\mathrm{c})=1$.

(ii) If $\left.\left.\mathrm{F}^{\prime}(\mathrm{c}) \in\right]-\infty, 0[\cup] 0,1\right]$, the characteristic equation admits a countable infinity of eigenvalues, all of them of negative real part, except the eigenvalue 0.

(iii) If $\mathrm{F}^{\prime}(\mathrm{c})>1$, there exists only one eigenvalue with positive real part, and it is a real solution. All other eigenvalues (in countable infinity) have negative real part, except the eigenvalue 0.

Proof. (i) If $\lambda$ is a multiple eigenvalue of the characteristic equation, then taking the first derivative we have $e^{\lambda}=\mathrm{F}^{\prime}(\mathrm{c})$ which implies $\lambda=\mathrm{F}^{\prime}(\mathrm{c})-1$ and thus $\lambda$ must be real in this case. This in turn leads to $\lambda=e^{\lambda}-1$, for which the only real solution is 0 : this proves Item (i).

To prove Items (ii) and (iii), let's write $\lambda=a+i b$ under real and imaginary parts $a, b$ in $\mathbb{R}$, and reformulate the characteristic equation as:

$$
\left\{\begin{array}{l}
a=\mathrm{F}^{\prime}(\mathrm{c})\left[1-e^{-a} \cos (b)\right] \\
b=\mathrm{F}^{\prime}(\mathrm{c}) e^{-a} \sin (b)
\end{array} .\right.
$$

(ii) Let's first assume $\mathrm{F}^{\prime}(\mathrm{c})<0$. We remark that any non null solution $\lambda=a+i b$ must satisfy $a<0$ (since we always have the relation $1-e^{-a} \cos (b) \geq 0$ for any $(a, b)$ in $\mathbb{R}_{+} \times \mathbb{R}$. 
Now, the real map $b \mapsto \ln \left(\frac{-\sin (b)}{b}\right)+b \operatorname{cotan}(b)$ is a surjective map (in fact even bijective) in each interval ] $k \pi,(k+1) \pi$ [, with $k$ odd in $\mathbb{Z}$ (since it tends to $+\infty$ in $\pi^{+}$and $-\infty$ in $2 \pi^{-}$) and therefore exists a countable infinity of values $b \in \mathbb{R}$ such that

$$
\ln \left(\frac{-\sin (b)}{b}\right)+b \operatorname{cotan}(b)=\mathrm{F}^{\prime}(\mathrm{c})-\ln \left(-\mathrm{F}^{\prime}(\mathrm{c})\right)
$$

Then we can set:

$$
a=\ln \left(\frac{-\sin (b)}{b}\right)+\ln \left(-\mathrm{F}^{\prime}(\mathrm{c})\right)
$$

from which comes:

$$
a=\mathrm{F}^{\prime}(\mathrm{c})\left(1-\frac{b \cos (b)}{\mathrm{F}^{\prime}(\mathrm{c}) \sin (b)}\right) \quad \text { and } \quad e^{a}=\mathrm{F}^{\prime}(\mathrm{c}) \frac{\sin (b)}{b}
$$

which means that $a+i b$ is solution. This proves that when $\mathrm{F}^{\prime}(\mathrm{c})<0$, there is an infinity of eigenvalues of the characteristic equation, and all of them have strictly negative real part (except the double eigenvalue 0 ).

Then let's assume $\left.\left.\mathrm{F}^{\prime}(\mathrm{c}) \in\right] 0,1\right]$ : in this case any non null solution $\lambda=a+i b$ must satisfy $a<0$ as well (otherwise we would have $|b|<|\sin (b)|$ which is absurd). By the exactly same reasoning as above, there exists an infinity of values $b \in \mathbb{R}$ such that

$$
\ln \left(\frac{\sin (b)}{b}\right)+b \operatorname{cotan}(b)=\mathrm{F}^{\prime}(\mathrm{c})-\ln \left(\mathrm{F}^{\prime}(\mathrm{c})\right)
$$

from which we conclude there exists as well an infinity of eigenvalues of the characteristic equation, all of them with strictly negative real part (except the eigenvalue 0). This proves Item (ii).

(iii) Lastly, to prove Item (iii) let's see $\mathrm{F}^{\prime}(\mathrm{c})$ as a parameter varying from a small positive value to infinity. By Item (ii), an eigenvalue with positive a can only emerge by intersecting or crossing the plane $\{a=0\}$ from below (i.e from negative values of $a$ ). But if $a=0$ then $b=0$ : therefore, as $\mathrm{F}^{\prime}(\mathrm{c})$ is varying, an eigenvalue with positive $a$ can only emerge from the multiple eigenvalue 0 . By Item (i), this means such a solution emerges at the value $\mathrm{F}^{\prime}(\mathrm{c})=1$. Since the multiplicity of 0 is two, it comes there exists a unique solution with positive real part for any given value $\mathrm{F}^{\prime}(\mathrm{c})>1$, and this is actually a real solution. As in (ii), there exists in addition to this positive solution, an infinity of eigenvalues with negative real part, which correspond to the ones existing for $\left.\mathrm{F}^{\prime}(\mathrm{c}) \in\right] 0,1\left[\right.$ that do not intersect the plane $\{a=0\}$ as $\mathrm{F}^{\prime}(\mathrm{c})$ crosses the value 1 . This proves Item (iii).

Now, among the constant solutions of Equation (16), those which are relevant for Equation (15) are given by the fixed points of $\mathrm{F}$ (as we will see in Lemma 6.5). Observe that F admits at least one fixed point $\omega^{*}$ such that $\mathrm{F}^{\prime}\left(\omega^{*}\right)<1$ since it is a periodic function from $\mathbb{R}$ to $\mathbb{R}$. Moreover, the larger $\eta \tau$ is, the more numerous these fixed points are, and the more numerous are the fixed points satisfying $\mathrm{F}^{\prime}\left(\omega^{*}\right)<1$ (see Figure 2 ). We have:

Proposition 6.4. For a generic choice of $\eta>0$ and $\tau>0$, the fixed points $\left(\omega_{1}^{*}, \cdots, \omega_{p}^{*}\right)$ of the function $\mathrm{F}=\eta \tau \mathrm{G}\left(\cdot+\theta_{\tau}\right)$ satisfy the condition $\mathrm{F}^{\prime}\left(\omega_{i}^{*}\right) \neq 0,1$.

Proof. For $x \in \mathbb{R}$, we have the equivalence:

$$
\left\{\begin{array} { l } 
{ \mathrm { F } ( x ) = x } \\
{ \mathrm { F } ^ { \prime } ( x ) \neq 0 , 1 }
\end{array} \Longleftrightarrow \left\{\begin{array}{l}
\mathrm{G}\left(x+\theta_{\tau}\right)=\frac{x}{\eta \tau} \\
\mathrm{G}^{\prime}\left(x+\theta_{\tau}\right) \neq 0, \frac{1}{\eta \tau}
\end{array}\right.\right.
$$

Then, consider a fixed point $\omega_{i}^{*}$ of $\mathrm{F}$ : if $\omega_{i}^{*}=0$, we clearly have $\mathrm{G}^{\prime}\left(\theta_{\tau}\right) \neq 0, \frac{1}{\eta \tau}$ for a generic choice of $\eta, \tau$ in $\mathbb{R}$. If, $\omega_{i}^{*}$ is non null, $\mathrm{G}\left(\omega_{i}^{*}+\theta\right)$ is non null as well, and a sufficient condition ensuring that $\omega_{i}^{*}$ satisfies $\mathrm{F}^{\prime}\left(\omega_{i}^{*}\right) \neq 0,1$ is that $\omega_{i}^{*}$ satisfies:

$$
\frac{\mathrm{G}^{\prime}\left(\omega_{i}^{*}+\theta_{\tau}\right)}{\mathrm{G}\left(\omega_{i}^{*}+\theta_{\tau}\right)} \neq 0, \frac{1}{\omega_{i}^{*}}
$$




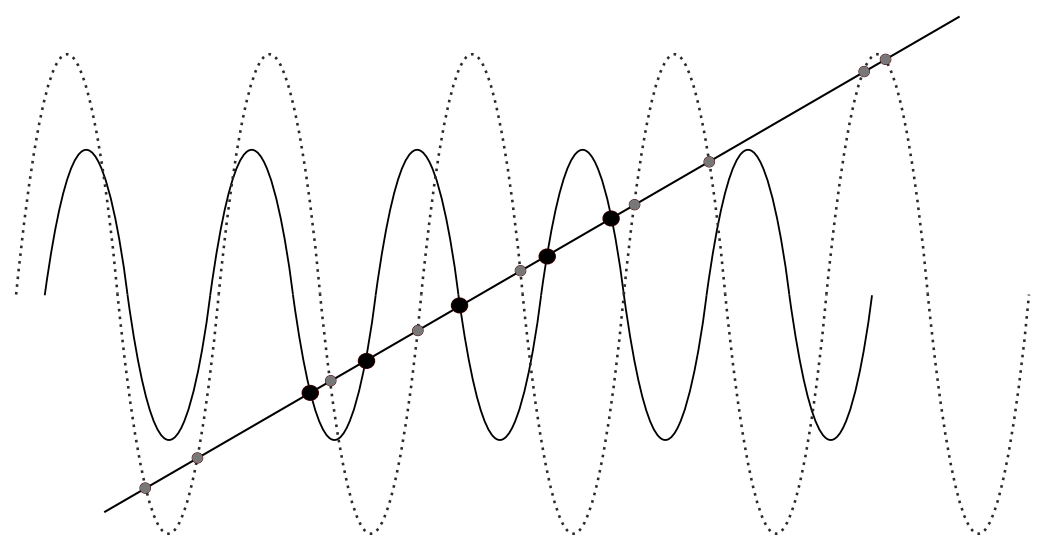

Figure 2. Fixed points of the $2 \pi$-periodic function $\mathrm{F}=\eta \tau \mathrm{G}\left(\cdot+\theta_{\tau}\right)$ : the number of fixed points increases with $\eta \tau$.

and so another sufficient condition is that $\omega_{i}^{*}$ satisfies:

$$
\frac{d}{d x}{ }_{\mid x=\omega_{i}^{*}} \ln \left(\left|\frac{\mathrm{G}\left(x+\theta_{\tau}\right)}{x}\right|\right) \neq 0 \text { and } \quad \frac{d}{d x}{ }_{\mid x=\omega_{i}^{*}} \ln \left(\left|\mathrm{G}\left(x+\theta_{\tau}\right)\right|\right) \neq 0 .
$$

In these last expressions, we can vary the parameter $\eta \in \mathbb{R}$ in a generic way so that the tangent at the curves of the maps $x \mapsto \ln \left(\left|\frac{\mathrm{G}\left(x+\theta_{\tau}\right)}{x}\right|\right)$ and $x \mapsto \ln \left(\left|\mathrm{G}\left(x+\theta_{\tau}\right)\right|\right)$ have a nonzero slope. Hence the result.

We know have, under this genericity condition of Proposition 6.4 (see the Appendix, Section 8.1, for a precise definition of normally hyperbolic invariant manifolds):

Lemma 6.5. Let $\eta>0, \tau>0$ and let $\mathcal{T}_{t, \eta, \tau}^{a v}$ the semi-flow associated to (15). Denote by $\left(\omega_{1}^{*}, \cdots, \omega_{p}^{*}\right)$ the fixed points of the function $\mathrm{F}=\eta \tau \mathrm{G}\left(\cdot+\theta_{\tau}\right)$. Then, there exists $\boldsymbol{\alpha}_{\mathbf{2}}>0$ such that for any $1 \leq i \leq p$, the following manifold of dimension two in $\mathscr{C}_{\boldsymbol{\alpha}_{2}}\left(\mathbb{R}_{-}, \mathbb{R}\right)^{2}$ :

$$
\mathcal{N}_{i, a v}=\left\{\left(\frac{2 \pi \tau}{T} \cdot+C_{1}, \omega_{i}^{*} \cdot+C_{2}\right), \text { where }\left(C_{1}, C_{2}\right) \in \mathbb{R}^{2}\right\}
$$

is invariant for the semi-flow $\mathcal{T}_{t, \eta, \tau}^{a v}$. Moreover, we have:

(i) If $\mathrm{F}^{\prime}\left(\omega_{i}^{*}\right)>1$, the manifold $\mathcal{N}_{\boldsymbol{i}, a v}$ is unstable and normally hyperbolic in $\mathscr{C}_{\boldsymbol{\alpha}_{\mathbf{2}}}\left(\mathbb{R}_{-}, \mathbb{R}\right)^{2}$, and its unstable manifold is of dimension 3 .

(ii) If $\mathrm{F}^{\prime}\left(\omega_{i}^{*}\right)<1$ and $\mathrm{F}^{\prime}\left(\omega_{i}^{*}\right) \neq 0$, the manifold $\mathcal{N}_{\boldsymbol{i}}$,av is stable and normally hyperbolic in $\mathscr{C}_{\boldsymbol{\alpha}_{\mathbf{2}}}\left(\mathbb{R}_{-}, \mathbb{R}\right)^{2}$.

Proof. Let us fix $\eta, \tau>0$ and $\alpha>0$ (the choice of $\alpha$ will be specified at the end of the proof).

Consider one of the manifolds $\mathcal{N}_{\boldsymbol{i} \text {,av }}$ associated to the fixed point $\omega_{i}^{*}$. Then, $\mathcal{N}_{\boldsymbol{i} \text {,av }}$ is invariant under $\mathcal{T}_{t, \eta, \tau}^{\text {av }}$. Indeed, we have, for any $t \geq 0$ :

$$
\frac{d}{d t}\left(\omega_{i}^{*} t+C_{2}\right)=\omega_{i}^{*}=\mathrm{F}\left[\omega_{i}^{*} t+C_{2}-\left(\omega_{i}^{*}(t-1)+C_{2}\right)\right]
$$

and thus for any $\left(C_{1}, C_{2}\right) \in \mathbb{R}^{2}$ and any $t \geq 0$ we have:

$$
\forall s \geq-t: \mathcal{T}_{t, \eta, \tau}^{\mathrm{av}}\left(\frac{2 \pi \tau}{T} \cdot+C_{1}, \omega_{i}^{*} \cdot+C_{2}\right)(s)=\left(\frac{2 \pi \tau}{T}(t+s)+C_{1}, \omega_{i}^{*}(t+s)+C_{2}\right),
$$

which proves the invariance of $\mathcal{N}_{i, \text { av }}$ under $\mathcal{T}_{t, \eta, \tau}^{\text {av }}$.

In order to prove Items (i) and (ii) we need to consider the linear semi-flow $D \mathcal{T}_{t, \eta, \tau}^{\mathrm{av}}(m)$ at each point $m=\left(\frac{2 \pi \tau}{T} \cdot+C_{1}, \omega_{i}^{*} \cdot+C_{2}\right) \in \mathcal{N}_{i, \text { av }}$. A simple writing of a rate of variation gives:

$$
\forall \beta, \phi \in \mathscr{C}_{\alpha}\left(\mathbb{R}_{-}, \mathbb{R}\right)^{2}, D \mathcal{T}_{t, \eta, \tau}^{\mathrm{av}}(m)(\beta, \phi)=\left(\beta(0), \mathcal{T}_{t, \omega_{i}^{*}}(\phi)\right),
$$


where $\beta(0)$ denotes the constant map of $\mathscr{C}_{\alpha}\left(\mathbb{R}_{-}, \mathbb{R}\right)$ equal to $\beta(0)$ on $\mathbb{R}_{-}$, and $\left.\mathcal{T}_{t, \omega_{i}^{*}}(\phi)\right)$ denotes the semi-flow associated to the linearized equation $\dot{\delta}=\mathrm{F}^{\prime}\left(\omega_{i}^{*}\right)(\delta(t)-\delta(t-1))$.

(i) To prove Item (i), let's consider the eigenvalues of the characteristic equation $-\lambda+\mathrm{F}^{\prime}\left(\omega_{i}^{*}\right)\left[1-e^{-\lambda}\right]=0$ : Since $\mathrm{F}^{\prime}\left(\omega_{i}^{*}\right)>1$ by assumption, following Proposition 6.3 , the eigenvalue $\lambda_{0}=0$ is simple, and we denote by $\lambda_{1}$ the unique positive eigenvalue, and $\left(\lambda_{n}\right)_{n \leq-1}$ all the eigenvalues with negative real part.

Now, it is a well-known fact on delay differential equations that the family of maps $\left\{s \mapsto e^{\lambda_{n} s}, n \leq 1\right\}$ forms a Schauder basis of the set $\mathscr{C}([-1,0], \mathbb{R})$. This means we have, for any $\phi \in \mathscr{C}([-1,0], \mathbb{R})$ :

$$
\forall s \in[-1,0]: \phi(s)=\sum_{n=-\infty}^{n=1} C_{n} e^{\lambda_{n} s}
$$

This decomposition is unfortunately not valid in $\mathscr{C}_{\alpha}\left(\mathbb{R}_{-}, \mathbb{R}\right)$, however thanks to it, we can still define the following projectors:

$$
\forall \phi \in \mathscr{C}_{\alpha}\left(\mathbb{R}_{-}, \mathbb{R}\right) \quad \begin{cases}p_{i}^{c}(\phi) & =C_{0} \\ p_{i}^{u}(\phi) & =C_{1} e^{\lambda_{1}}\end{cases}
$$

where $C_{0}$ and $C_{1}$ are the constants associated to the eigenvalues $\lambda_{0}, \lambda_{1}$ in the decomposition of $\phi_{\mid[-1,0]}$ in the Schauder basis, and where $e^{\lambda_{1}} \cdot$ denotes the map $s \mapsto e^{\lambda_{1} s}$ from $\mathbb{R}_{-}$to $\mathbb{C}$. Then, we can set:

$$
\left\{\begin{array}{l}
E_{m}^{c}=\mathbb{R}^{2} \\
E_{m}^{u}=\left\{\left(0, C_{1} e^{\lambda_{1} \cdot}\right), C_{1} \in \mathbb{R}\right\} \\
E_{m}^{s}=\left\{\left(\beta-\beta(0), \phi-p_{i}^{c}(\phi)-p_{i}^{u}(\phi)\right),(\beta, \phi) \in \mathscr{C}_{\alpha}\left(\mathbb{R}_{-}, \mathbb{R}\right)^{2}\right\}
\end{array}\right.
$$

where $e^{\lambda_{1}} \cdot$ denotes the map $s \mapsto e^{\lambda_{1} s}$ from $\mathbb{R}_{-}$to $\mathbb{C}$. These three sets are closed subspaces of $\mathscr{C}_{\alpha}\left(\mathbb{R}_{-}, \mathbb{R}\right)^{2}$ (where $\mathbb{R}$ is identified as the set of constant functions over $\mathbb{R}_{-}$and is therefore seen as a subspace of $\left.\mathscr{C}_{\alpha}\left(\mathbb{R}_{-}, \mathbb{R}\right)\right)$ and we observe:

$$
\begin{cases}\forall\left(C_{1}, C_{2}\right) \in \mathbb{R}^{2}, & D \mathcal{T}_{t, \eta, \tau}^{\mathrm{av}}(m)\left(C_{1}, C_{2}\right)=\left(C_{1}, C_{2}\right) \\ \forall C_{1} \in \mathbb{R}, & D \mathcal{T}_{t, \eta, \tau}^{\text {av }}(m)\left(0, C_{1} e^{\lambda_{1} \cdot}\right)=\left(0, C_{1} e^{\lambda_{1} t} e^{\lambda_{1} \cdot}\right)\end{cases}
$$

and also that we have, for any $s \in \mathbb{R}_{-}$:

$$
\begin{aligned}
D \mathcal{T}_{t, \eta, \tau}^{\mathrm{av}}(m)\left(\beta-\beta(0), \phi-p_{i}^{c}(\phi)-p_{i}^{u}(\phi)\right)(s) & =\left\{\begin{array}{l}
\left(0, \phi(t+s)-p_{i}^{c}(\phi)(t+s)-p_{i}^{u}(\phi)(t+s)\right), \text { if } s \leq-t-1 \\
\left(0, \sum_{n=-\infty}^{-1} C_{n} e^{\lambda_{n}(t+s)}\right), \text { if } s \geq-t-1
\end{array}\right. \\
& =\left(0, \mathcal{T}_{t, \omega_{i}^{*}}(\phi)(s)-p_{i}^{c}\left(\mathcal{T}_{t, \omega_{i}^{*}}(\phi)\right)(s)-p_{i}^{u}\left(\mathcal{T}_{t, \omega_{i}^{*}}(\phi)\right)(s)\right) .
\end{aligned}
$$

Therefore the linearized semi-flow satisfies: $D \mathcal{T}_{t, \eta, \tau}^{\text {av }}(m)_{\mid E_{m}^{\alpha}}: E_{m}^{\alpha} \rightarrow E_{m_{1}}^{\alpha}=E_{m}^{\alpha}$ (for $\alpha=c, u, s$ ) where $m_{1}=\mathcal{T}_{t, \eta, \tau}^{\mathrm{av}}(m)=\left(\frac{2 \pi \tau}{T}(t+\cdot)+C_{1}, \omega_{i}^{*}(t+\cdot)+C_{2}\right)$. Using the relation:

$$
\left\{\begin{array}{l}
\beta=\beta(0)+[\beta-\beta(0)] \\
\phi=p_{i}^{c}(\phi)+p_{i}^{u}(\phi)+\left[\phi-p_{i}^{c}(\phi)-p_{i}^{u}(\phi)\right]
\end{array}\right.
$$

we thus obtain:

$$
\mathscr{C}_{\alpha}\left(\mathbb{R}_{-}, \mathbb{R}\right)^{2}=E_{m}^{c} \oplus E_{m}^{u} \oplus E_{m}^{s}
$$

in which we remark that $E_{m}^{c}=T_{m} \mathcal{N}_{i \text {,av }}$. The unstable space $E_{m}^{u}$ at $m$ is of dimension 1, which automatically gives us that $D \mathcal{T}_{t, \eta, \tau}^{\text {av }}(m)_{\mid E_{m}^{u}}$ is an isomorphism from $E_{m}^{u}$ to $E_{m_{1}}^{u}$. To conclude that $\mathcal{N}_{\boldsymbol{i} \text {,av }}$ is a normally hyperbolic invariant manifold, it remains to check (see Definition of Section 8.1 in the Appendix) that $D \mathcal{T}_{t, \eta, \tau}^{\text {av }}(m)$ is expansive on $E_{m}^{u}$ and contractive on $E_{m}^{s}$, at a rate greater than its behavior on the tangent space $E_{m}^{c}$. In this purpose we easily notice:

$$
\left\{\begin{array}{l}
\min \left[1, \inf \left\{\left\|D \mathcal{T}_{t, \eta, \tau}^{\mathrm{av}}(m)(\beta, \phi)\right\|_{\alpha},(\beta, \phi) \in E_{m}^{c},\|(\beta, \phi)\|_{\alpha}=1\right\}\right]=1 \\
\inf \left\{\left\|D \mathcal{T}_{t, \eta, \tau}^{\mathrm{av}}(m)(\beta, \phi)\right\|_{\alpha},(\beta, \phi) \in E_{m}^{u},\|(\beta, \phi)\|_{\alpha}=1\right\}=e^{\lambda_{1} t}
\end{array}\right.
$$


The contractivity of $D \mathcal{T}_{t, \eta, \tau}^{\text {av }}(m)_{\mid E_{m}^{s}}$ is more technical to obtain: to do it, let's fix $(\beta, \phi) \in E_{m}^{s}$ with expression for $\phi$ over $[-1,0]$ given by $\phi(s)=\sum_{n=-\infty}^{n=-1} C_{n} e^{\lambda_{n} s}$. Then we have, for any $m \leq-1$ :

$$
\begin{aligned}
\int_{-1}^{0} \phi(s) e^{-\lambda_{m} s} d s & =\sum_{n=-\infty}^{n=-1} C_{n} \int_{-1}^{0} \phi(s) e^{\left(\lambda_{n}-\lambda_{m}\right) s} d s \\
& =C_{m}+\sum_{n \neq m} C_{n} e^{\lambda_{m}}\left[\frac{e^{-\lambda_{m}}-e^{-\lambda_{n}}}{\lambda_{n}-\lambda_{m}}\right] \\
& =C_{m}+\frac{e^{\lambda_{m}}}{\mathrm{~F}^{\prime}\left(\omega_{i}^{*}\right)} \sum_{n \neq m} C_{n} \\
& =C_{m}\left(1-\frac{e^{\lambda_{m}}}{\mathrm{~F}^{\prime}\left(\omega_{i}^{*}\right)}\right)+\frac{e^{\lambda_{m}}}{\mathrm{~F}^{\prime}\left(\omega_{i}^{*}\right)} \phi(0) \\
& =C_{m}\left(1-\frac{1}{\mathrm{~F}^{\prime}\left(\omega_{i}^{*}\right)-\lambda_{m}}\right)+\frac{1}{\mathrm{~F}^{\prime}\left(\omega_{i}^{*}\right)-\lambda_{m}} \phi(0) .
\end{aligned}
$$

For any $m \leq-1$, the eigenvalue $\lambda_{m}$ is in $\mathbb{C} \backslash \mathbb{R}$ which ensures $\mathrm{F}^{\prime}\left(\omega_{i}^{*}\right)-\lambda_{m}-1 \neq 0$. From this we get:

$$
\forall m \leq-1, C_{m}=\frac{\mathrm{F}^{\prime}\left(\omega_{i}^{*}\right)-\lambda_{m}}{\mathrm{~F}^{\prime}\left(\omega_{i}^{*}\right)-\lambda_{m}-1} \int_{-1}^{0} \phi(s) e^{-\lambda_{m} s} d s-\frac{1}{\mathrm{~F}^{\prime}\left(\omega_{i}^{*}\right)-\lambda_{m}-1} \phi(0) .
$$

As seen in the proof of Proposition 6.3, we have $\left|\lambda_{m}\right| \sim|m|$ when $m$ tends to infinity, and so there exists a constant $K>0$ such that:

$$
\forall m \leq-1:\left|C_{m}\right| \leq K \sup _{s \in[-1,0]}|\phi(s)|
$$

Now, for any $t \geq 2$, we have $D \mathcal{T}_{t, \eta, \tau}^{\mathrm{av}}(m)(\beta-\beta(0), \phi)(s)=\left(0, \sum_{n=-\infty}^{n=-1} C_{n} e^{\lambda_{n}(t+s)}\right)$ and since $D \mathcal{T}_{t, \eta, \tau}^{\text {av }}(m)$ is a compact operator on $\mathscr{C}([-1,0], \mathbb{R})^{2}$, the map $D \mathcal{T}_{t, \eta, \tau}^{\text {av }}(m)(\beta-\beta(0), \phi)$ is twice differentiable over $[-1,0]$. This allows us to write:

$$
\forall t \geq 2, \forall s \in[-1,0]: \quad \frac{d^{2}}{d s^{2}} D \mathcal{T}_{t, \eta, \tau}^{\mathrm{av}}(m)(\beta-\beta(0), \phi)(s)=\left(0, \sum_{n=-\infty}^{n=-1} C_{n} \lambda_{n}^{2} e^{\lambda_{n}(t+s)}\right),
$$

and applying the inequality (17) above, we get:

$$
\exists K>0, \forall t \geq 2, \forall s \in[-1,0]: \sum_{n=-\infty}^{-1}\left|C_{n}\right| \leq K \sup _{s \in[-1,0]}\left\|\frac{d^{2}}{d s^{2}} D \mathcal{T}_{t, \eta, \tau}^{\mathrm{av}}(m)(\beta-\beta(0), \phi)(s)\right\|
$$

and by compactness again of $D \mathcal{T}_{t, \eta, \tau}^{\text {av }}(m)$ on $\mathscr{C}([-1,0], \mathbb{R})^{2}$, we get the estimation:

$$
\begin{gathered}
\exists K>0, \forall s \in[-1,0]: \sum_{n=-\infty}^{-1}\left|C_{m}\right| \leq K \sup _{s \in[-1,0]}|| D \mathcal{T}_{2, \eta, \tau}^{\mathrm{av}}(m)(\beta-\beta(0), \phi)(s) \| \\
\leq K K_{2} \sup _{s \in[-1,0]}|\phi(s)|,
\end{gathered}
$$

where $K_{2}$ denotes the norm of the operator $D \mathcal{T}_{2, \eta, \tau}^{\text {av }}(m)$ on $\mathscr{C}([-1,0], \mathbb{R})^{2}$.

From this estimation we finally obtain, for any $\boldsymbol{\alpha}_{\mathbf{2}}>0$ satisfying $\Re\left(\lambda_{n}\right)+\boldsymbol{\alpha}_{\mathbf{2}}<0$ (for any $n \leq-1$ ):

$$
\begin{aligned}
\forall t \geq 2, \forall(\beta, \phi) \in E_{m}^{s},\left\|D \mathcal{T}_{t, \eta, \tau}^{\mathrm{av}}(m)(\beta-\beta(0), \phi)\right\|_{\boldsymbol{\alpha}_{\mathbf{2}}} & \leq e^{-\boldsymbol{\alpha}_{\mathbf{2}} t} \max \left(\|\phi\|_{\boldsymbol{\alpha}_{\mathbf{2}}}, \sum_{n=-\infty}^{n=-1}\left|C_{n}\right|\right) \\
& \leq e^{-\boldsymbol{\alpha}_{\mathbf{2}} t} \max \left(\left\|\phi\left|\|_{\boldsymbol{\alpha}_{\mathbf{2}}}, K K_{2} e^{\boldsymbol{\alpha}_{\mathbf{2}}} \sup _{s \in[-1,0]}\right| \phi(s) \mid\right)\right. \\
& \leq e^{-\boldsymbol{\alpha}_{\mathbf{2}} t} \max \left(1, K K_{2} e^{\boldsymbol{\alpha}_{\mathbf{2}}}\right)\|\phi\|_{\boldsymbol{\alpha}_{\mathbf{2}} .}
\end{aligned}
$$


We thus can conclude that exist a time $t_{0} \geq 2$ and a real number $\mu<1$ such that we have, for any $t \geq t_{0}$ :

$$
\begin{aligned}
& \mu \inf \left\{\left\|D \mathcal{T}_{t, \eta, \tau}^{\mathrm{av}}(m)(\beta, \phi)\right\|_{\boldsymbol{\alpha}_{\mathbf{2}}},(\beta, \phi) \in E_{m}^{u},\|(\beta, \phi)\|_{\boldsymbol{\alpha}_{\mathbf{2}}}=1\right\}>1 \\
& \mu>\left\|D \mathcal{T}_{t, \eta, \tau}^{\mathrm{av}}(m)_{\mid E_{m}^{s}}\right\|_{\boldsymbol{\alpha}_{\mathbf{2}}}
\end{aligned}
$$

which ends the proof that $\mathcal{N}_{\boldsymbol{i} \text {, av }}$ is a normally hyperbolic invariant manifold in $\mathscr{C}_{\boldsymbol{\alpha}_{\mathbf{2}}}\left(\mathbb{R}_{-}, \mathbb{R}\right)^{2}\left(\right.$ where $\boldsymbol{\alpha}_{\mathbf{2}}>0$ satisfies $\Re\left(\lambda_{n}\right)+\boldsymbol{\alpha}_{\mathbf{2}}<0$ for any $\left.n \leq-1\right) . \mathcal{N}_{\boldsymbol{i} \text {, av }}$ is unstable and since $\operatorname{dim}\left(E_{m}^{u}\right)=1$, therefore the dimension of the unstable manifold associated to $\mathcal{N}_{\boldsymbol{i} \text {, av }}$ is: $\operatorname{dim}\left(T_{m} \mathcal{N}_{\boldsymbol{i} \text {,av }}\right)+\operatorname{dim}\left(E_{m}^{u}\right)=3$, as desired. We have proved Item (i).

(ii) The proof of Item (ii) is completely similar.

Lemma 6.6. Let $\eta>0, \tau>0$ such that the condition $\mathrm{F}^{\prime}\left(\omega_{i}^{*}\right) \neq 0,1$ be satisfied for the fixed points $\omega_{1}^{*}, \cdots, \omega_{p}^{*}$ of $\mathrm{F}$. Then, the union set $\cup_{i=1}^{p} \mathcal{N}_{\boldsymbol{i}, \text { av }}$ is a globally stable (invariant) manifold in $\mathscr{C}_{\boldsymbol{\alpha}_{\mathbf{2}}}\left(\mathbb{R}_{-}, \mathbb{R}\right)^{2}\left(\right.$ where $\boldsymbol{\alpha}_{\mathbf{2}}$ is chosen as in the proof of Lemma 6.5) for the semi-flow $\mathcal{T}_{t, \eta, \tau}^{a v}$ of Equation (15), in the following sense:

$$
\begin{aligned}
& \forall(\beta, \phi) \in \mathscr{C}_{\boldsymbol{\alpha}_{\mathbf{2}}}\left(\mathbb{R}_{-}, \mathbb{R}\right)^{2}, \exists i \in\{1, \cdots, p\}, \exists\left(C_{1}, C_{2}\right) \in \mathbb{R}^{2}: \\
& \sup _{s \in[-1,0]}\left\|\mathcal{T}_{t, \eta, \tau}^{a v}(\beta, \phi)(s)-\mathcal{T}_{t, \eta, \tau}^{a v}\left(\frac{2 \pi \tau}{T} \cdot+C_{1}, \omega_{i}^{*} \cdot+C_{2}\right)(s)\right\| \rightarrow 0
\end{aligned}
$$

when the time t tends to infinity.

This means that the union $\cup_{i=1}^{p} \mathcal{N}_{\boldsymbol{i} \text {, av }}$ is "attracting" all initial conditions in $\mathscr{C}_{\boldsymbol{\alpha}_{\mathbf{2}}}\left(\mathbb{R}_{-}, \mathbb{R}\right)^{2}$ for the semi-flow of Equation (15).

This means also that the only invariant manifolds for the semi-flow of Equation (15), are the $\mathcal{N}_{\boldsymbol{i} \text {,av }}$.

Proof. Let us fix $\left(\beta_{0}, \phi_{0}\right) \in \mathscr{C}_{\boldsymbol{\alpha}_{\mathbf{2}}}\left(\mathbb{R}_{-}, \mathbb{R}\right)^{2}$ and write for any $t \geq 0$ and $s \leq 0, \mathcal{T}_{t, \eta, \tau}^{\text {av }}\left(\beta_{0}, \phi_{0}\right)(s)=\left(\frac{2 \pi \tau}{T}(t+s)+\right.$ $\left.\beta_{0}(0), \phi(t+s)\right)$, that is to say we denote by $\phi(t+s)$ the solution of the equation $\dot{\phi}^{\text {new }}(t)=\eta \tau \mathrm{G}\left[\phi^{\text {new }}(t)-\right.$ $\left.\phi^{\text {new }}(t-1)+\theta_{\tau}\right]$ with initial condition $\phi_{0}$, taken at time $t+s$. Let's also set $\omega(t)=\phi(t)-\phi(t-1)$, for any $t \geq 0$.

Then, $\omega$ is differentiable for $t \geq 1$, and so $\dot{\omega}(t)=\mathrm{F}(\omega(t))-\mathrm{F}(\omega(t-1))$, which means $\omega$ is solution of Equation (16): by Lemma 6.1 , it comes that $\omega(t)$ converges asymptotically to a constant $C$. On the other hand, for any $t \geq 1$, there exists $c_{t} \in[t-1, t]$ such that: $\omega(t)=\dot{\phi}\left(c_{t}\right)=\mathrm{F}\left(\omega\left(c_{t}\right)\right)$ : therefore the constant $C$ must be one of the fixed points of the map F, say $\omega_{i}^{*}$.

Now, as in Lemma 6.5 , each open interval of the set $\left\{c \in \mathbb{R}: \mathrm{F}^{\prime}(\mathrm{c}) \neq 0,1\right\}$ is a one-dimensional normally hyperbolic invariant manifold of $\mathscr{C}_{\boldsymbol{\alpha}_{\mathbf{2}}}\left(\mathbb{R}_{-}, \mathbb{R}\right)$ for the semi-flow of (16): this can be shown in an exactly similar way as the proof of Lemma 6.5 (and this since the linearized semi-flow of (16) at c has a similar expression as the one of $(15)$ at any point $\left.\left(\frac{2 \pi \tau}{T} \cdot+C_{1}, c \cdot+C_{2}\right)\right)$. Therefore, the convergence of the trajectory $\omega$ towards such a component of $\left\{c \in \mathbb{R}: \mathrm{F}^{\prime}(\mathrm{c}) \neq 0,1\right\}$ containing $\omega_{i}^{*}$ happens exponentially fast (since this trajectory must enter the stable manifold associated to this normally hyperbolic invariant manifold). This means there exist two constants $K>0, a>0$, and a time $t_{0} \geq 1$, such that the inequality $\left|\omega(t)-\omega_{i}^{*}\right| \leq K e^{-a t}$ holds, for any $t \geq t_{0}$. We have:

$$
\begin{aligned}
\forall t \geq t_{0} \geq 1,\left(\phi(t)-\omega_{i}^{*} t\right)-\left(\phi\left(t_{0}\right)-\omega_{i}^{*} t_{0}\right) & =\int_{t_{0}}^{t}\left(\frac{d \phi}{d u}(u)-\omega_{i}^{*}\right) d u \\
& =\int_{t_{0}}^{t}\left(\mathrm{~F}(\omega(u))-\mathrm{F}\left(\omega_{i}^{*}\right)\right) d u .
\end{aligned}
$$

It comes (since the function $\mathrm{F}^{\prime}$ is bounded):

$$
\int_{t_{0}}^{+\infty}\left|\mathrm{F}(\omega(s))-\mathrm{F}\left(\omega_{i}^{*}\right)\right| e^{a s} e^{-a s} d s \leq \frac{K}{a} \cdot \sup _{t \in \mathbb{R}+}\left|\mathrm{F}^{\prime}(t)\right|<+\infty,
$$


and therefore the integral $\int_{t_{0}}^{+\infty}\left(\mathrm{F}(\omega(u))-\mathrm{F}\left(\omega_{i}^{*}\right)\right) d u$ is convergent. Setting

$$
C_{0}=\phi\left(t_{0}\right)-\omega_{i}^{*} t_{0}+\int_{t_{0}}^{+\infty}\left(\mathrm{F}(\omega(u))-\mathrm{F}\left(\omega_{i}^{*}\right)\right) d u,
$$

we have obtained that the constant $C_{0}$ does not depend on the time $t_{0}$ (but only depends on the initial condition $\phi_{0}$ in $\left.\mathscr{C}_{\boldsymbol{\alpha}_{\mathbf{2}}}\left(\mathbb{R}_{-}, \mathbb{R}\right)\right)$ and that:

$$
\lim _{t \rightarrow \infty} \phi(t)-\omega_{i}^{*} t=C_{0}
$$

From this, we conclude we have:

$$
\begin{aligned}
& \forall\left(\beta_{0}, \phi_{0}\right) \in \mathscr{C}_{\boldsymbol{\alpha}_{\mathbf{2}}}\left(\mathbb{R}_{-}, \mathbb{R}\right)^{2}, \exists i \in\{1, \cdots, p\}, \exists C_{0} \in \mathbb{R}: \\
& \sup _{s \in[-1,0]}\left\|\mathcal{T}_{t, \eta, \tau}^{\mathrm{av}}\left(\beta_{0}, \phi_{0}\right)(s)-\mathcal{T}_{t, \eta, \tau}^{\mathrm{av}}\left(\frac{2 \pi \tau \cdot}{T}+\beta_{0}(0), \omega_{i}^{*} \cdot+C_{0}\right)(s)\right\| \rightarrow 0
\end{aligned}
$$

as desired.

The result of this Lemma means that any trajectory enters (after a finite time) the stable manifold of one of the normally hyperbolic invariant manifolds of $\mathcal{T}_{t, \eta, \tau}^{\text {av }}$, say $\mathcal{N}_{\boldsymbol{i} \text {,av }}$, whether this is a stable or unstable manifold.

Corollary 6.7. Let us fix $L_{1}, L_{2} \geq 1$. Then, there exists $\eta_{0}>0$ such that for a generic choice of $\eta, \tau$ in the set $\left\{(\eta, \tau) \in \mathbb{R}^{2}: 0<\eta<\eta_{0}, \tau>0, L_{1} \leq \eta \tau \leq L_{2}\right\}$, the $\mathscr{C}^{1}$ semi-flow $\mathcal{T}_{(8), t, \eta, \tau}$ of Equation (8) admits the following normally hyperbolic invariant manifolds:

$$
\mathcal{N}_{i}=\left\{\left(\frac{2 \pi \tau}{T}+C_{1}, \omega_{i}^{*} \cdot+C_{2}+\alpha_{i}\left(\cdot, \eta, \tau, C_{1}, C_{2}\right)\right), \text { where }\left(C_{1}, C_{2}\right) \in \mathbb{R}^{2}\right\}, \quad i=1, \cdots, p
$$

in $\mathscr{C}_{\boldsymbol{\alpha}_{\mathbf{2}}}\left(\mathbb{R}_{-}, \mathbb{R}\right)^{2}$ (with $\boldsymbol{\alpha}_{\mathbf{2}}>0$ taken as in Lemma 6.5), where the perturbation $s \mapsto \alpha_{i}\left(s, \eta, \tau, C_{1}, C_{2}\right)$ tends to 0 when $\eta$ tends to 0 (uniformly in $\tau, C_{1}, C_{2}$ ), depends smoothly in $C_{1}, C_{2}$ and satisfy the relations:

$$
\begin{aligned}
\alpha_{i}\left(\cdot, \eta, \tau, C_{1}, C_{2}\right) & =\alpha_{i}\left(\cdot, \eta, \tau, 0, C_{1}+C_{2}\right) \\
\alpha_{i}(\cdot, \eta, \tau, 0, C+2 \pi) & =\alpha_{i}(\cdot, \eta, \tau, 0, C),
\end{aligned}
$$

for any $\left(C_{1}, C_{2}, C\right) \in \mathbb{R}^{3}$. The union of these manifolds satisfy:

$$
\begin{aligned}
& \forall(\beta, \phi) \in \mathscr{C}_{\boldsymbol{\alpha}_{\mathbf{2}}}\left(\mathbb{R}_{-}, \mathbb{R}\right)^{2}, \exists i \in\{1, \cdots, p\}, \exists\left(C_{1}, C_{2}\right) \in \mathbb{R}^{2}: \\
& \sup _{s \in[-1,0]}\left\|\mathcal{T}_{(8), t, \eta, \tau}(\beta, \phi)(s)-\mathcal{T}_{(8), t, \eta, \tau}\left(\frac{2 \pi \tau \cdot}{T}+C_{1}, \omega_{i}^{*} \cdot+C_{2}+\alpha_{i}\left(\cdot, \eta, \tau, C_{1}, C_{2}\right)\right)(s)\right\| \rightarrow 0
\end{aligned}
$$

when the time $t$ tends to infinity.

Proof. Let's first consider Equation (13). For $\eta \tau$ larger than a constant $L \geq 1$, this equation is a $\mathscr{C}^{1}$ perturbation, of order of magnitude $\eta$, of Equation (15): in this perturbation, the variable $\beta$ is not affected, and this perturbation tends to 0 uniformly in $\tau$, when $\eta$ tends to 0 . Therefore, applying Lemma 6.6 and the result of Bates, Lu, Zeng for $\mathscr{C}^{1}$ semi-flows on Banach spaces (see the theorem of Section 8.1 in the Appendix), we automatically get the persistence of the manifolds $\mathcal{N}_{\mathbf{1}}$, av $, \cdots, \mathcal{N}_{p \text {,av }}$ for the semiflow of (13). Thus, given $L_{1}, L_{2} \geq 1$, exists $\eta_{0}>0$ and a function $\left(s, C_{1}, C_{2}\right) \mapsto \alpha_{i}\left(s, \eta, \tau, C_{1}, C_{2}\right) \in \mathbb{R}$ which tends to 0 when $\eta$ tends to 0 (uniformly in $\tau, C_{1}, C_{2}$ ) such that, for a generic choice of $\eta, \tau$ in $\left\{(\eta, \tau) \in \mathbb{R}^{2}: 0<\eta<\eta_{0}, \tau>0, L_{1} \leq \eta \tau \leq L_{2}\right\}$, each $\mathcal{N}_{i}$ with the form claimed is a normally hyperbolic invariant manifold for the semi-flow of (13), is of same stability as $\mathcal{N}_{\boldsymbol{i}}$,av , and is unique (in a neighborhood of size $\eta$ of $\mathcal{N}_{\boldsymbol{i} \text {,av }}$ ). Similarly for the semi-flow of (8), since the variable $\phi^{\text {new }}$ is a perturbation of $\phi$ which tends to 0 uniformly in $s$ and $\tau$, when $\eta$ tends to 0.

Now since the semi-flow of (15) admits no other invariant set than the normally hyperbolic invariant manifolds $\mathcal{N}_{i \text {,av }}$, it comes (by normal hyperbolicity) that for a generic choice of $\eta, \tau$ in $\left\{(\eta, \tau) \in \mathbb{R}^{2}: 0<\eta<\right.$ $\left.\eta_{0}, \tau>0, L_{1} \leq \eta \tau \leq L_{2}\right\}$, the semi-flow $\mathcal{T}_{(8), t, \eta, \tau}$ of System (8) has no other invariant set than the $\mathcal{N}_{\boldsymbol{i}}$.

So, as for Equation (15), for any initial condition $\left(\beta_{0}, \phi_{0}\right) \in \mathscr{C}_{\boldsymbol{\alpha}_{\mathbf{2}}}\left(\mathbb{R}_{-}, \mathbb{R}\right)^{2}$ the trajectory $\left(\mathcal{T}_{(8), t, \eta, \tau}\left(\beta_{0}, \phi_{0}\right)\right)_{t \geq 0}$ enters the stable manifold of one of the manifolds $\mathcal{N}_{\boldsymbol{i}}$ (whether it is a stable or unstable manifold) after a 
finite time. Hence the global stability claim.

Lastly, we observe that Equation (8) is invariant under the transformation $K \mapsto(\beta+K, \phi-K)$ where $K$ is a real variable. This means that given any initial condition $\left(\beta_{0}, \phi_{0}\right) \in \mathscr{C}_{\boldsymbol{\alpha}_{\mathbf{2}}}\left(\mathbb{R}_{-}, \mathbb{R}\right)^{2}$, the curve $(\beta(t), \phi(t))_{t \geq 0}$ solution of (8) with initial condition $\left(\beta_{0}, \phi_{0}\right)$ satisfies that $(\beta+K, \phi-K)$ is still a solution of (8). Thus, taking the limit of $\left(\mathcal{T}_{(8), t, \eta, \tau}\left(\beta_{0}, \phi_{0}\right)\right)_{t \geq 0}$ when $t$ tends to infinity, we obtain easily that for any $\left(K, C_{1}, C_{2}\right) \in \mathbb{R}^{3}$,

$$
\begin{aligned}
& \left(\frac{2 \pi \tau \cdot}{T}+K+C_{1}, \omega_{i}^{*} \cdot+C_{2}-K+\alpha_{i}\left(\cdot, \eta, \tau, C_{1}, C_{2}\right)\right) \\
= & \left(\frac{2 \pi \tau \cdot}{T}+K+C_{1}, \omega_{i}^{*} \cdot+C_{2}-K+\alpha_{i}\left(\cdot, \eta, \tau, K+C_{1}, C_{2}-K\right)\right) .
\end{aligned}
$$

Taking $K=-C_{1}$ it comes that, for any $\left(C_{1}, C_{2}\right) \in \mathbb{R}^{2}$ :

$$
\alpha_{i}\left(\cdot, \eta, \tau, 0, C_{1}+C_{2}\right)=\alpha_{i}\left(\cdot, \eta, \tau, C_{1}, C_{2}\right) .
$$

Moreover, since the equation (8) is defined by a function $\mathrm{h}$ which is $2 \pi$-periodic in the variable $\psi$, it also comes:

$$
\forall\left(\beta_{0}, \phi_{0}\right) \in \mathscr{C}_{\boldsymbol{\alpha}_{\mathbf{2}}}\left(\mathbb{R}_{-}, \mathbb{R}\right)^{2}, \mathcal{T}_{(8), t, \eta, \tau}\left(\beta_{0}, \phi_{0}-2 \pi\right)=\mathcal{T}_{(8), t, \eta, \tau}\left(\beta_{0}, \phi_{0}\right)-(0,2 \pi),
$$

from which we deduce the relation:

$$
\alpha_{i}\left(\cdot, \eta, \tau, 0, C_{1}+C_{2}+2 \pi\right)=\alpha_{i}\left(\cdot, \eta, \tau, 0, C_{1}+C_{2}\right),
$$

as desired.

\section{END OF THE PROOF OF THE MAIN RESUlT}

From Corollary 6.7 we can now come back to Equation (6) and establish the following result:

Corollary 7.1. Let us fix $L_{1}, L_{2} \geq 1$. Then, there exists $\eta_{0}>0$ such that for a generic choice of $\eta, \tau$ in the set $\left\{(\eta, \tau) \in \mathbb{R}^{2}: 0<\eta<\eta_{0}, \tau>0, L_{1} \leq \eta \tau \leq L_{2}\right\}$, there exist $p$ real numbers $\left(\omega_{1}^{*}, \cdots, \omega_{p}^{*}\right) \in \mathbb{R}^{p}$ and $2 p$ functions:

$$
\left\{\begin{array}{ll}
(s, C) & \mapsto \alpha_{i}(s, \eta, \tau, C) \\
\mathbb{R}_{-} \times \mathbb{R} & \rightarrow \mathbb{R},
\end{array}, \quad \begin{cases}(s, C) & \mapsto \beta_{i}(s, \eta, \tau, C) \\
\mathbb{R}_{-} \times \mathbb{R} & \rightarrow \mathbb{R},\end{cases}\right.
$$

for which the following holds.

For any initial condition $\psi_{0} \in \mathscr{C}_{\boldsymbol{\alpha}_{\mathbf{1}}}\left(\mathbb{R}_{-}, \mathbb{R}\right)$, (where $\boldsymbol{\alpha}_{\mathbf{1}}$ is chosen as in Theorem 4.5) there exist $C \in \mathbb{R}$ and $1 \leq i \leq p$ such that the trajectory $\left(\mathcal{T}_{\mathcal{M}_{\alpha_{1}}, t, \eta, \tau}\left(\psi_{0}\right)\right)_{t \geq 0}$ solution of Equation (6) starting at $\psi_{0}$ satisfies:

$$
\lim _{t \rightarrow+\infty} \sup _{s \in[-1,0]}\left|\mathcal{T}_{\mathcal{M}_{\boldsymbol{\alpha}_{1}}, t, \eta, \tau}\left(\psi_{0}\right)(s)-\mathcal{T}_{\mathcal{M}_{\boldsymbol{\alpha}_{\mathbf{1}}}, t, \eta, \tau}\left(\chi_{i, C}\right)(s)\right|=0
$$

where $\chi_{i, C}$ is defined by:

$$
\chi_{i, C}=\frac{2 \pi \tau \cdot}{T}+\omega_{i}^{*} \cdot+C+\alpha_{i}(\cdot, \eta, \tau, C)+\beta_{i}(\cdot, \eta, \tau, C) .
$$

The two functions $s \mapsto \alpha_{i}(s, \eta, \tau, C)$ and $s \mapsto \beta_{i}(s, \eta, \tau, C)$ tend to 0 when $\eta$ tends to 0 (uniformly in $\tau, C$ ), and satisfy the relation:

$$
\begin{aligned}
& \alpha_{i}(\cdot, \eta, \tau, C+2 \pi)=\alpha_{i}(\cdot, \eta, \tau, C) \\
& \beta_{i}(\cdot, \eta, \tau, C+2 \pi)=\beta_{i}(\cdot, \eta, \tau, C) .
\end{aligned}
$$

Proof. Consider the system:

$$
\left\{\begin{array}{l}
\dot{\beta}=\frac{2 \pi \tau}{T} \\
\dot{\phi}=\eta \tau \mathcal{G}_{1}\left(\phi_{t}+\beta_{t}\right)+\eta^{2} \tau \mathcal{H}_{\boldsymbol{\eta}}\left(\phi_{t}+\beta_{t}\right)
\end{array}\right.
$$


which is System (6) extended to $\mathbb{R}^{2}$ by introducing the fast variable $\beta$, as we did for System (8). Then the semi-flow $\mathcal{T}_{(18), t, \eta, \tau}$ of System $(18)$ is $\mathscr{C}^{1}$ on $\mathscr{C}_{\alpha}\left(\mathbb{R}_{-}, \mathbb{R}\right.$ ) (for any arbitrary given $\alpha>0$ ) and is a $\mathscr{C}^{1}$ perturbation of System (8) of order of magnitude $\eta^{2} \tau$. Therefore, applying Corollary 6.7 and applying the persistence result of Bates, Lu, Zeng (i.e the theorem of Section 8.1 in the Appendix) for $\mathscr{C}^{1}$ semi-flows on Banach spaces, we obtain that:

$$
\begin{aligned}
& \forall\left(\beta_{0}, \phi_{0}\right) \in \mathscr{C}_{\boldsymbol{\alpha}_{\mathbf{2}}}\left(\mathbb{R}_{-}, \mathbb{R}\right)^{2}, \exists i \in\{1, \cdots, p\}, \exists\left(C_{1}, C_{2}\right) \in \mathbb{R}^{2}, \forall s \in[-1,0]: \\
& \left\|\mathcal{T}_{(18), t, \eta, \tau}\left(\beta_{0}, \phi_{0}\right)(s)-\mathcal{T}_{(18), t, \eta, \tau}\left(\frac{2 \pi \tau \cdot}{T}+C_{1}, \omega_{i}^{*} \cdot+C_{2}+\alpha_{i}\left(\cdot, \eta, \tau, 0, C_{1}+C_{2}\right)+\beta_{i}\left(\cdot, \eta, \tau, 0, C_{1}+C_{2}\right)\right)(s)\right\|
\end{aligned}
$$

tends to 0 when the time $t$ tends to infinity, in which the terms $\alpha_{i}\left(\cdot, \eta, \tau, 0, C_{1}+C_{2}\right)$ and $\beta_{i}\left(\cdot, \eta, \tau, 0, C_{1}+C_{2}\right)$ are small terms of order $\eta^{2} \tau$ and satisfy the claimed properties of periodicity. Observing that for any $\psi_{0} \in \mathscr{C}_{\boldsymbol{\alpha}_{\mathbf{1}}}\left(\mathbb{R}_{-}, \mathbb{R}\right) \cap \mathscr{C}_{\boldsymbol{\alpha}_{\mathbf{2}}}\left(\mathbb{R}_{-}, \mathbb{R}\right)$, the term $\mathcal{T}_{\mathcal{M}_{\boldsymbol{\alpha}_{1}}, t, \eta, \tau}\left(\psi_{0}\right)$ can be written as the sum of the first and second component of $\mathcal{T}_{(18), t, \eta, \tau}\left(\beta_{0}, \psi_{0}-\beta_{0}\right)$, we get the result for elements $\psi_{0} \in \mathscr{C}_{\boldsymbol{\alpha}_{1}}\left(\mathbb{R}_{-}, \mathbb{R}\right) \cap \mathscr{C}_{\boldsymbol{\alpha}_{2}}\left(\mathbb{R}_{-}, \mathbb{R}\right)$.

Finally, given any $\psi_{0} \in \mathscr{C}_{\boldsymbol{\alpha}_{\mathbf{1}}}\left(\mathbb{R}_{-}, \mathbb{R}\right)$, it suffices to consider an element $\tilde{\psi}_{0} \in \mathscr{C}_{\boldsymbol{\alpha}_{\mathbf{1}}}\left(\mathbb{R}_{-}, \mathbb{R}\right) \cap \mathscr{C}_{\boldsymbol{\alpha}_{\mathbf{2}}}(\mathbb{R}-, \mathbb{R})$ satisfying:

$$
\tilde{\psi}_{0 \mid[-1,0]}=\psi_{0 \mid[-1,0]}
$$

and to notice that:

$$
\forall t \geq 0, \forall s \in[-1,0], \mathcal{T}_{\mathcal{M}_{\boldsymbol{\alpha}_{1}}, t, \eta, \tau}\left(\tilde{\psi}_{0}\right)(s)=\mathcal{T}_{\mathcal{M}_{\boldsymbol{\alpha}_{1}}, t, \eta, \tau}\left(\psi_{0}\right)(s)
$$

to get the result.

Remark 7.2. Observe that the persistence of normally hyperbolic invariant manifolds argument that we applied in this proof gives us, as for Corollary 6.7, the following relation:

$$
\forall 1 \leq i \leq p, \forall C \in \mathbb{R}, \forall t \geq 0, \exists C_{t} \in \mathbb{R},: \mathcal{T}_{\mathcal{M}_{\alpha_{1}}, t, \eta, \tau}\left(\chi_{i, C}\right)=\chi_{i, C+C_{t}},
$$

which is as well an invariance relation.

From Corollary 7.1, we can now use Theorem 4.5 (on the global stability of the manifold $\mathcal{M}_{\boldsymbol{\alpha}_{1}}$ associated to the $\mathscr{C}^{2}$ function $\mathrm{G}_{\boldsymbol{\alpha}_{1}}$ ), from which it directly comes the following theorem, which is the main result of this paper expressed in terms of the variables $(\psi, y)$, i.e for System $(2)$ :

Theorem 7.3. There exists $\delta_{0}>0$ for which, given $L_{1}, L_{2} \geq 1$, there exists $\eta_{0}>0$ such that for a generic choice of $\eta, \tau$ in the set $\left\{(\eta, \tau) \in \mathbb{R}^{2}: 0<\eta<\eta_{0}, \tau>0, L_{1} \leq \eta \tau \leq L_{2}\right\}$, there exist $\boldsymbol{\alpha}_{\mathbf{1}}>0$, p real numbers $\omega_{1}^{*}, \cdots, \omega_{p}^{*} \in \mathbb{R}^{p}$ and $p$ functions of class $\mathscr{C}^{1}$ :

$$
\begin{cases}(s, C) & \mapsto \gamma_{i}(s, \eta, \tau, C) \\ \mathbb{R}_{-} \times \mathbb{R} & \rightarrow \mathbb{R},\end{cases}
$$

for which the following holds.

For any initial condition $\left(\psi_{0}, y_{0}\right) \in \mathscr{C}([-\tau, 0], \mathbb{R}) \times \mathcal{B}_{\delta_{0} / 2}\left([-\tau, 0], \mathbb{R}^{n-1}\right)$, there exist $C \in \mathbb{R}$ and $1 \leq i \leq p$ such that the trajectory $\left(\mathcal{T}_{(2), t, \eta, \tau}\left(\psi_{0}, y_{0}\right)\right)_{t \geq 0}$ solution of System $(2)$ starting at $\left(\psi_{0}, y_{0}\right)$ satisfies:

$$
\lim _{t \rightarrow+\infty} \sup _{s \in[-\tau, 0]}\left\|\mathcal{T}_{(2), t+\tau, \eta, \tau}\left(\psi_{0}, y_{0}\right)(s)-\mathcal{T}_{(2), t, \eta, \tau}\left(\chi_{i, C}, \mathrm{G}_{\boldsymbol{\alpha}_{\mathbf{1}}}\left(\chi_{i, C}\right)\right)(s)\right\|=0
$$

where $\mathrm{G}_{\boldsymbol{\alpha}_{1}}$ is a $\mathscr{C}^{2}$ function on $\left(\mathscr{C}_{\boldsymbol{\alpha}_{\mathbf{1}}}\left(\mathbb{R}_{-}, \mathbb{R}\right),\|\cdot\|_{\boldsymbol{\alpha}_{1}}\right)$ and $\chi_{i, C}$ is defined by:

$$
\chi_{i, C}=\frac{1}{\tau}\left[\frac{2 \pi \tau \cdot}{T}+\omega_{i}^{*} \cdot\right]+C+\gamma_{i}\left(\frac{1}{\tau} \cdot, \eta, \tau, C\right) .
$$

The function $\gamma_{i}(\cdot, \eta, \tau, C)$ tends to 0 when $\eta$ tends to 0 (uniformly in $\tau, C$ ) and for any $(C, t) \in \mathbb{R} \times \mathbb{R}_{+}$, there exists $C_{t} \in \mathbb{R}$ such that:

$$
\begin{aligned}
\gamma_{i}(\cdot, \eta, \tau, C+2 \pi) & =\gamma_{i}(\cdot, \eta, \tau, C) \\
\mathcal{T}_{(2), t, \eta, \tau}\left(\chi_{i, C}, \mathrm{G}_{\boldsymbol{\alpha}_{\mathbf{1}}}\left(\chi_{i, C}\right)\right) & =\left(\chi_{i, C+C_{t}}, \mathrm{G}_{\boldsymbol{\alpha}_{\mathbf{1}}}\left(\chi_{i, C+C_{t}}\right)\right) .
\end{aligned}
$$


Proof. Let's take $\delta_{0}, \eta_{0}>0$ satisfying Theorem 4.5, and fix $L_{1}, L_{2} \geq 1$. Reducing $\eta_{0}$ if necessary we can assume $\eta_{0}$ satisfies Corollary 7.1. Let's take a generic choice of $\eta, \tau$ in $\left\{(\eta, \tau) \in \mathbb{R}^{2}: 0<\eta<\eta_{0}, \tau>0, L_{1} \leq\right.$ $\left.\eta \tau \leq L_{2}\right\}$ and $\boldsymbol{\alpha}_{\mathbf{1}}>0$ satisfying Theorem 4.5.

Then, given $\left(\psi_{0}, y_{0}\right) \in \mathscr{C}([-1,0], \mathbb{R}) \times \mathcal{B}_{\delta_{0} / 2}\left([-1,0], \mathbb{R}^{n-1}\right)$, Theorem 4.5 tells us that there exists $\left(\psi_{1}, y_{\psi_{1}}\right) \in$ $\mathcal{M}_{\alpha_{1}}$ such that:

$$
\lim _{t \rightarrow+\infty} \sup _{s \in[-1,0]}\left\|\mathcal{T}_{(3), t+1, \eta, \tau}\left(\psi_{0}, y_{0}\right)(s)-\mathcal{T}_{(3), t, \eta, \tau}\left(\psi_{1}, y_{\psi_{1}}\right)(s)\right\|=0,
$$

where $\mathcal{T}_{(3), t, \eta, \tau}$ denotes the semi-flow associated to System (3).

Now, setting $\gamma_{i}(\cdot, \eta, \tau, C)=\alpha_{i}(\cdot, \eta, \tau, C)+\beta_{i}(\cdot, \eta, \tau, C)$ (where the two terms $\alpha_{i}(\cdot, \eta, \tau, C)$ and $\beta_{i}(\cdot, \eta, \tau, C)$ are of order $\eta^{2} \tau$ and taken as in Corollary 7.1) and applying Corollary 7.1, there exist $1 \leq i \leq p$ and $C \in \mathbb{R}$ such that:

$$
\lim _{t \rightarrow+\infty} \sup _{s \in[-1,0]}\left|\mathcal{T}_{\mathcal{M}_{\boldsymbol{\alpha}_{1}}, t, \eta, \tau}\left(\psi_{1}\right)(s)-\mathcal{T}_{\mathcal{M}_{\boldsymbol{\alpha}_{1}}, t, \eta, \tau}\left(\chi_{i, C}\right)(s)\right|=0 .
$$

By construction of the manifold $\mathcal{M}_{\boldsymbol{\alpha}_{1}}$, we have:

$$
\begin{aligned}
\mathcal{T}_{(3), t, \eta, \tau}\left(\psi_{1}, y_{\psi_{1}}\right) & =\left(\mathcal{T}_{\mathcal{M}_{\boldsymbol{\alpha}_{\mathbf{1}}, t, \eta, \tau}}\left(\psi_{1}\right), \mathrm{G}_{\boldsymbol{\alpha}_{1}}\left(\mathcal{T}_{\mathcal{M}_{\boldsymbol{\alpha}_{1}}, t, \eta, \tau}\left(\psi_{1}\right)\right)\right) \\
\mathcal{T}_{(3), t, \eta, \tau}\left(\chi_{i, C}, \mathrm{G}_{\boldsymbol{\alpha}_{\mathbf{1}}}\left(\chi_{i, C}\right)\right) & =\left(\mathcal{T}_{\mathcal{M}_{\boldsymbol{\alpha}_{1}}, t, \eta, \tau}\left(\chi_{i, C}\right), \mathrm{G}_{\boldsymbol{\alpha}_{\mathbf{1}}}\left(\mathcal{T}_{\mathcal{M}_{\boldsymbol{\alpha}_{1}}, t, \eta, \tau}\left(\chi_{i, C}\right)\right)\right)
\end{aligned}
$$

and so, for any $t \geq 0$, for any $s \in[-1,0]$ :

$$
\begin{aligned}
& \left\|\mathcal{T}_{(3), t, \eta, \tau}\left(\psi_{1}, y_{\psi_{1}}\right)(s)-\mathcal{T}_{(3), t, \eta, \tau}\left(\chi_{i, C}, \mathrm{G}_{\boldsymbol{\alpha}_{\mathbf{1}}}\left(\chi_{i, C}\right)\right)(s)\right\| \\
= & \left\|\left(\mathcal{T}_{\mathcal{M}_{\boldsymbol{\alpha}_{1}, t, \eta, \tau}}\left(\psi_{1}\right)(s)-\mathcal{T}_{\mathcal{M}_{\boldsymbol{\alpha}_{1}, t, \eta, \tau}}\left(\chi_{i, C}\right)(s), \mathrm{G}_{\boldsymbol{\alpha}_{\mathbf{1}}}\left(\mathcal{T}_{\mathcal{M}_{\boldsymbol{\alpha}_{1}}, t, \eta, \tau}\left(\psi_{1}\right)\right)(s)-\mathrm{G}_{\boldsymbol{\alpha}_{\mathbf{1}}}\left(\mathcal{T}_{\mathcal{M}_{\boldsymbol{\alpha}_{1}, t, \eta, \tau}}\left(\chi_{i, C}\right)\right)(s)\right)\right\|,
\end{aligned}
$$

from which we get:

$$
\lim _{t \rightarrow+\infty} \sup _{s \in[-1,0]}\left\|\mathcal{T}_{(3), t, \eta, \tau}\left(\psi_{1}, y_{\psi_{1}}\right)(s)-\mathcal{T}_{(3), t, \eta, \tau}\left(\chi_{i, C}, \mathrm{G}_{\boldsymbol{\alpha}_{\mathbf{1}}}\left(\chi_{i, C}\right)\right)(s)\right\|=0,
$$

which gives the result for the semi-flow $\mathcal{T}_{(3), t, \eta, \tau}$ of System (3).

Rescaling the time by the factor $\tau$, we get the result for trajectories $\left(\mathcal{T}_{(2), t, \eta, \tau}\left(\psi_{0}, y_{0}\right)\right)_{t \geq 0}$ of System (2) starting at initial conditions $\left(\psi_{0}, y_{0}\right) \in \mathscr{C}([-\tau, 0], \mathbb{R}) \times \mathcal{B}_{\delta_{0} / 2}\left([-\tau, 0], \mathbb{R}^{n-1}\right)$.

Finally, from Theorem 7.3 and Lemma 2.2, we can now deduce the main result of this paper:

Proof of Theorem $A$. We fix $L_{1}, L_{2} \geq 1$ and take $\delta_{0}>0, \eta_{0}>0$ satisfying the claim of Theorem 7.3, and (reducing their value if necessary) of Lemma 2.2. We also take a generic choice of $\eta, \tau$ in the set $\left\{(\eta, \tau) \in \mathbb{R}^{2}: 0<\eta<\eta_{0}, \tau>0, L_{1} \leq \eta \tau \leq L_{2}\right\}$.

(i) First, let's take an integer $1 \leq i \leq p$, and $C \in \mathbb{R}$. By Theorem (7.3) we have:

$$
\forall t \geq 0, \exists C_{t} \in \mathbb{R}, \mathcal{T}_{(2), t, \eta, \tau}\left(\chi_{i, C}, \mathrm{G}_{\boldsymbol{\alpha}_{\mathbf{1}}}\left(\chi_{i, C}\right)\right)=\left(\chi_{i, C+C_{t}}, \mathrm{G}_{\boldsymbol{\alpha}_{\mathbf{1}}}\left(\chi_{i, C+C_{t}}\right)\right) .
$$

Since we have:

$$
\forall t \geq 0: \mathcal{T}_{(2), t, \eta, \tau}\left(\chi_{i, C}, \mathrm{G}_{\boldsymbol{\alpha}_{\mathbf{1}}}\left(\chi_{i, C}\right)\right)(-t)=\mathcal{T}_{(2), 0, \eta, \tau}\left(\chi_{i, C}, \mathrm{G}_{\boldsymbol{\alpha}_{\mathbf{1}}}\left(\chi_{i, C}\right)\right)(0),
$$

it thus comes for any $t \geq 0$ :

$$
C_{t}-\left(\frac{2 \pi}{T} t+\frac{\omega_{i}^{*}}{\tau} t\right)=\gamma_{i}(0, \eta, \tau, C)-\gamma_{i}\left(\frac{-t}{\tau}, \eta, \tau, C+C_{t}\right)
$$

Since $\gamma_{i}(\cdot, \eta, \tau, C)$ is in $\left(\mathscr{C}_{\boldsymbol{\alpha}_{1}}\left(\mathbb{R}_{-}, \mathbb{R}\right),\|\cdot\|_{\boldsymbol{\alpha}_{1}}\right)$ and of order $\eta^{2} \tau$, exists a constant $K$ such that:

$$
\left|C_{\tau}-\tau\left(\frac{2 \pi}{T}+\eta G\left(\omega_{i}^{*}+\theta_{\tau}\right)\right)\right| \leq K\left(1+e^{1}\right) \eta^{2} \tau,
$$

from which we deduce, since $C_{0}=0$, the existence of a time $t=A_{i}$ such that $C_{A_{i}}=2 \pi$ (which time $A_{i}$ is actually $\eta$-close to $T$ ). Notice that $A_{i}$ also depends on the value $C$ which has been initially fixed: to avoid cumbersome notations, we don't write this dependence on $C$ but we keep it in mind for the end of the proof. Now, we observe that the map $\mathcal{T}_{(2), A_{i}, \eta, \tau}\left(\chi_{i, C}, \mathrm{G}_{\boldsymbol{\alpha}_{\mathbf{1}}}\left(\chi_{i, C}\right)\right)$ belonging to $\mathscr{C}_{\boldsymbol{\alpha}_{\mathbf{1}}}\left(\mathbb{R}_{-}, \mathbb{R}\right)$ is in fact of class $\mathscr{C}^{1}$ 
over $\left[-A_{i}, 0\right]$ (since the semi-flow $\mathcal{T}_{(2), A_{i}, \eta, \tau}$ is of class $\mathscr{C}^{1}$ over $\left.\mathscr{C}\left(\left[-A_{i}, 0\right], \mathbb{R}\right)\right)$. This gives that $\gamma_{i}\left(\frac{\dot{-}}{\tau}, \eta, \tau, C\right)$ is also $\mathscr{C}^{1}$ over $\left[-A_{i}, 0\right]$, and then it comes:

$$
\left|A_{i}-\frac{2 \pi}{\frac{2 \pi}{T}+\frac{\omega_{i}^{*}}{\tau}}\right| \leq K_{1} \frac{A_{i}}{\tau} \frac{1}{\frac{2 \pi}{T}+\frac{\omega_{i}^{*}}{\tau}} \eta^{2} \tau
$$

in which $K_{1}>0$ is a constant. Thus exists a constant $M_{0}>0$ such that:

$$
\left|A_{i}-\frac{2 \pi}{\frac{2 \pi}{T}+\frac{\omega_{i}^{*}}{\tau}}\right| \leq M_{0} \eta^{2}
$$

as claimed. By construction of the manifold $\mathcal{M}_{\boldsymbol{\alpha}_{1}}$, we have $\mathrm{G}_{\boldsymbol{\alpha}_{1}}(\psi+2 \pi)=\mathrm{G}_{\boldsymbol{\alpha}_{1}}(\psi)$ for any $\psi \in \mathscr{C}_{\boldsymbol{\alpha}_{1}}\left(\mathbb{R}_{-}, \mathbb{R}\right)$ (see Lemma 4.3), from which comes, by properties of $\gamma_{i}(\cdot, \eta, \tau, C)$ given by Theorem 7.3:

$$
\begin{aligned}
\mathcal{T}_{(2), A_{i}, \eta, \tau}\left(\chi_{i, C}, \mathrm{G}_{\boldsymbol{\alpha}_{\mathbf{1}}}\left(\chi_{i, C}\right)\right) & =\left(\chi_{i, C+2 \pi}, \mathrm{G}_{\boldsymbol{\alpha}_{\mathbf{1}}}\left(\chi_{i, C+2 \pi}\right)\right) \\
& =\left(\chi_{i, C+2 \pi}, \mathrm{G}_{\boldsymbol{\alpha}_{\mathbf{1}}}\left(\chi_{i, C}\right)\right)
\end{aligned}
$$

and so we obtain the equality:

$$
\mathcal{T}_{(2), t+A_{i}, \eta, \tau}\left(\chi_{i, C}, \mathrm{G}_{\boldsymbol{\alpha}_{\mathbf{1}}}\left(\chi_{i, C}\right)\right)=\mathcal{T}_{(2), t, \eta, \tau}\left(\chi_{i, C}, \mathrm{G}_{\boldsymbol{\alpha}_{\mathbf{1}}}\left(\chi_{i, C}\right)\right)+(2 \pi, 0)
$$

where here 0 denotes the null point in $\mathbb{R}^{n-1}$.

(ii) Then, to obtain the desired result from this equality, it remains to apply Lemma 2.2 which makes the correspondance between curves solutions of System (2) in the variables $(\psi, y)$ and solutions of our original System (1) in the variable $x$.

We take an initial condition $\gamma \in \mathcal{V}_{\Gamma, \tau}\left(\delta_{0} / 2\right)$. By definition of the diffeomorphism $\Phi$ of Lemma 2.2, the curve $(\psi(t), y(t))_{t \geq 0}$ starting at the initial condition $\Phi \circ \gamma_{0}$ satisfies:

$$
\forall t \geq 0, \forall s \in[-1,0]: \mathcal{T}_{t, \eta, \tau}(\gamma)(s)=\Phi^{-1}(\psi(t+s), y(t+s)) .
$$

By Theorem 7.3, there exist an integer $1 \leq i \leq p$ and $C \in \mathbb{R}$ such that:

$$
\sup _{s \in[-1,0]}\left\|(\psi(t+s+1), y(t+s+1))-\mathcal{T}_{(2), t, \eta, \tau}\left(\chi_{i, C}, \mathrm{G}_{\boldsymbol{\alpha}_{\mathbf{1}}}\left(\chi_{i, C}\right)\right)(s)\right\| \rightarrow 0
$$

when the time $t$ tends to infinity. Therefore we have:

$$
\sup _{s \in[-1,0]}\left\|\mathcal{T}_{t+1, \eta, \tau}(\gamma)(s)-\Phi^{-1}\left(\mathcal{T}_{(2), t, \eta, \tau}\left(\chi_{i, C}, \mathrm{G}_{\boldsymbol{\alpha}_{\mathbf{1}}}\left(\chi_{i, C}\right)\right)(s)\right)\right\| \rightarrow 0 .
$$

Observing that $\Phi^{-1}\left(\mathcal{T}_{(2), t+A_{i}, \eta, \tau}\left(\chi_{i, C}, \mathrm{G}_{\boldsymbol{\alpha}_{\mathbf{1}}}\left(\chi_{i, C}\right)\right)(s)\right)=\Phi^{-1}\left(\mathcal{T}_{(2), t, \eta, \tau}\left(\chi_{i, C}, \mathrm{G}_{\boldsymbol{\alpha}_{\mathbf{1}}}\left(\chi_{i, C}\right)\right)(s)\right)$ for any $t \geq 0$ and for any $s \in[-1,0]$ (since $\Phi$ maps into $\mathbb{S}^{1} \times \mathbb{R}^{n-1}$ ), we can finally conclude that the trajectory $\left(\Phi^{-1} \circ\right.$ $\left.\mathcal{T}_{(2), t, \eta, \tau}\left(\chi_{i, C}, \mathrm{G}_{\boldsymbol{\alpha}_{\mathbf{1}}}\left(\chi_{i, C}\right)\right)\right)_{t \geq 0}$ is a periodic orbit (of period $A_{i}=A_{i}(C)$ close to $\frac{2 \pi}{\frac{2 \pi}{T}+\frac{\omega_{i}^{*}}{\tau}}$ ), towards which the trajectory $\left(\mathcal{T}_{t+1, \eta, \tau}(\gamma)\right)_{t \geq 0}$ converges asymptotically. In fact, this orbit $\left(\Phi^{-1} \circ \mathcal{T}_{(2), t, \eta, \tau}\left(\chi_{i, C}, \mathrm{G}_{\boldsymbol{\alpha}_{1}}\left(\chi_{i, C}\right)\right)\right)_{t \geq 0}$ is the same as the orbit $\left(\Phi^{-1} \circ \mathcal{T}_{(2), t, \eta, \tau}\left(\chi_{i, 0}, \mathrm{G}_{\boldsymbol{\alpha}_{1}}\left(\chi_{i, 0}\right)\right)\right)_{t \geq 0}$ (again since $\Phi$ maps into $\mathbb{S}^{1} \times \mathbb{R}^{n-1}$ ), which is a periodic orbit of period $A_{i}(0)$. Therefore, there are only $p$ distinct such periodic orbits, whose periods $A_{i}$ do not depend on $C$. QED.

\section{Conclusion}

We have rigorously proved a self-induced synchronization result for Equation (1), resulting from the perturbation of an arbitrary vector field $\mathrm{f}$ of class $\mathscr{C}^{r}, r \geq 3$, in $\mathbb{R}^{n}$ (around a stable periodic orbit of $\mathrm{f}$ ), by a delayed feedback term $\eta \mathrm{g}(x, x(t-\tau))$ of same regularity. The result obtained (Theorem A) showed that trajectories asymptotically enter into a periodic regime and are split in (a finite number of) clusters defined by frequencies equal (at first order in $\eta$ ) to $\frac{2 \pi}{T}+\frac{\omega_{i}^{*}}{\tau}$. Such a result was proved for a generic choice of the parameters $\eta, \tau$, under the assumption that $\eta>0$ be small, $\tau>0$ be large and $\eta \tau$ be bounded by a constant 
larger than one: it remains to describe the dynamics when there is no more this restriction on $\eta \tau$ (possibly unbounded).

Our synchronization result often appears in the context of networks (notably coupled phase oscillator networks), where very often it is stated for averaged systems reduced from the original delay equation, or in the case where $\eta \tau$ is small ([22], [1]): we will apply the result we presented here to dynamical networks in a future paper.

Acknowledgements. The author warmly thanks Dmitry Turaev who strongly contributed to this work and to the setting of this problem, and without whom this paper would never have seen the day, and Tiago Pereira as well. This work was co-funded by the EPSRC Centre for Predictive Modelling in Healthcare grant EP/N014391/1, and by the FAPESP BEPE Grant 17/21896-3 thanks to which the author could visit Dmitry Turaev during 6 months in the dynamical systems group at Imperial College London. The author is also in debt to Jan Sieber from the University of Exeter for very useful discussions on delay dynamical systems, and Jaap Eldering for discussions about Normally Hyperbolic Invariant Manifolds.

\section{APPENDIX}

8.1. Persistence of Normally Hyperbolic Invariant Manifolds for semi-flows in Banach spaces. Let us first recall the precise definition of a normally hyperbolic invariant manifold (see [3]):

Definition (Bates, Lu, Zeng, see [3]). Let $(X,|\cdot|)$ be a Banach space, equipped with the linear operator norm $\|\cdot\|$, and $M$ an invariant manifold for a semi-flow $T_{t}: X \rightarrow X$ of class $\mathscr{C}^{1}$ for any $t \geq 0$. Then, $M$ is a normally hyperbolic invariant manifold for $T_{t}$ if:

(H1) For each $m \in M$ there is a decomposition $X=X_{m}^{c} \oplus X_{m}^{u} \oplus X_{m}^{s}$ of closed subspaces with $X_{m}^{c}$ the tangent space to $M$ at $m$.

(H2) For each $m \in M$ and $t \geq 0$, if $m_{1}=T_{t}(m)$ :

$$
D T_{t}(m)_{\mid X_{m}^{\alpha}}: X_{m}^{\alpha} \rightarrow X_{m_{1}}^{\alpha}, \text { for } \alpha=c, u, s
$$

and $D T_{t}(m)_{\mid X_{m}^{u}}$ is an isomorphism from $X_{m}^{u}$ onto $X_{m_{1}}^{u}$.

(H3) There exists $t_{0} \geq 0$ and $\lambda<1$ such that for all $t \geq t_{0}$

$$
\begin{aligned}
& \lambda \inf \left\{\left|D T_{t}(m)\left(x^{u}\right)\right|: x^{u} \in X_{m}^{c},\left|x^{u}\right|=1\right\}>\max \left\{1,\left\|D T_{t}(m)_{\mid X_{m}^{c}}\right\|\right\} \\
& \lambda \min \left\{1, \inf \left\{\left|D T_{t}(m)\left(x^{c}\right)\right|: x^{c} \in X_{m}^{c},\left|x^{c}\right|=1\right\}\right\}>\left\|D T_{t}(m)_{\mid X_{m}^{s}}\right\| .
\end{aligned}
$$

As said in [3], Condition (H3) essentially asks that near $m \in M$, the hyperbolic dynamics in the direction normal to $M$ is much stronger than the dynamics on $M$ itself: i.e, the map $T_{t}$ is expansive in the direction of $X_{m}^{u}$, and at a rate greater than that on $M$, and $T_{t}$ is contractive in the direction of $X_{m}^{s}$ and at a rate greater than that on $M$.

With this definition we can now reproduce the persistence result of Bates, Lu, Zeng (first stated in [3] for $\mathscr{C}^{1}$ semi-flows on $\mathscr{C}^{2}$ manifolds, and then improved in [5] by a stronger version for $\mathscr{C}^{1}$ manifolds) that we use at several points in this paper:

Theorem (Bates, Lu, Zeng, see [3]). Let $X$ a Banach space, $T_{t}$ a $\mathscr{C}^{1}$ semi-flow of $X$, and $M$ a $\mathscr{C}^{2}$ compact connected invariant manifold on which $T_{t}$ is normally hyperbolic for $t$ sufficiently large. Suppose also that for each $m \in M, D T_{t \mid X_{m}^{u}}$ is an isomorphism. Let $t_{1}>0$ be large enough and be fixed and $N$ be a fixed neighborhood of $M$. For any $\epsilon>0$, there exists $\sigma>0$ such that if $\tilde{T}$ is a $\mathscr{C}^{1}$ map on $X$ which satisfies $\left\|\tilde{T}-T_{t_{1}}\right\|_{\mathscr{C}^{1}(N)}<\sigma$, then:

(a) Persistence: $\tilde{T}$ has a unique $\mathscr{C}^{1}$ compact connected normally hyperbolic invariant manifold $\tilde{M}$ near $M$.

(b) Convergence: $\tilde{M}$ converges to $M$ in the $\mathscr{C}^{1}$ topology as $\left\|\tilde{T}-T_{t_{1}}\right\|_{\mathscr{C}^{1}(N)}$ tends zero.

(c) Existence: $\tilde{T}$ has unique $\mathscr{C}^{1}$ invariant manifolds $\tilde{W}^{c s}(\epsilon)$ and $\tilde{W}^{c u}(\epsilon)$ in a tubular neighborhood $N(\epsilon)$ of $M$, which at $M$ are tangent to the center-stable vector bundle $\tilde{X}^{c} \oplus \tilde{X}^{s}$ and the center-unstable vector bundle $\tilde{X}^{c} \oplus \tilde{X}^{u}$, respectively.

(d) Characterization: $\tilde{W}^{c s}(\epsilon)=\left\{x_{0} \in N(\epsilon): \tilde{T}^{k}\left(x_{0}\right) \in N(\epsilon)\right.$, for $k \geq 1, \tilde{T}^{k}\left(x_{0}\right) \rightarrow \tilde{M}$ as $\left.k \rightarrow+\infty\right\}$ $\tilde{W}^{c u}(\epsilon)=\left\{x_{0} \in N(\varepsilon): \exists\left(x_{k}\right)_{k \geq 0} \subset N(\epsilon)\right.$, satisfying $\tilde{T}\left(x_{k}\right)=x_{k-1}$ for $k \geq 1$, and $x_{k} \rightarrow \tilde{M}$ as $\left.k \rightarrow+\infty\right\}$ 
and $\tilde{M}=\tilde{W}^{c u}(\epsilon) \cap \tilde{W}^{c s}(\epsilon)$.

Furthermore, if $\tilde{T}_{t}$ is a $\mathscr{C}^{1}$ semiflow on $X$ which satisfies $\left\|\tilde{T}_{t_{1}}-T_{t_{1}}\right\|_{\mathscr{C}^{1}(N)}<\sigma$ and $\left\|\tilde{T}_{t}-T_{t}\right\|_{\mathscr{C}^{0}(N)}<\sigma$ for $0 \leq t \leq t_{1}$, then $\tilde{M}$ is a normally hyperbolic invariant manifold for $\tilde{T}_{t}$ with center-stable manifold $\tilde{W}^{\text {cs }}(\epsilon)$ and center-unstable manifold $\tilde{W}^{\text {cu }}(\epsilon)$ respectively.

The reader might have observed that this persistence result is set for compact normally hyperbolic invariant manifolds: however, as said in [4], this compactness assumption can be released provided the quantities in the estimates above are uniform (which is the case in Lemma 6.5). It is the stronger statement of [5] (with the $\mathscr{C}^{2}$ requirement on $M$ relaxed to $\mathscr{C}^{1}$ ) together with this uniform estimates assumption (replacing compactness) that we use all along the paper.

\subsection{Basic estimates involving the matrix $A$.}

Lemma 8.1. Let $A$ be a matrix in $\mathcal{M}_{n}(\mathbb{R})$ whose eigenvalues are all strictly negative. Then, there exist $\sigma>0, C>0$ and a matrix $P \in G L_{n}(\mathbb{R})$ such that the following holds:

(ii) For any $z \in \mathbb{R}^{n}:\left\langle z, P^{-1} A P z\right\rangle \leq-\sigma\|z\|$.

(ii) For any $t>0$ : $\left\|e^{A t}\right\| \leq C e^{-\sigma t}$.

Proof. See [35], or [8].

\subsection{Proof of Theorem 4.5 (global stability of the manifold $\mathcal{M}_{\alpha_{1}}$ ).}

Proof. As for the proof of Lemma 4.3, we apply the Perron-Lyapunov method, adapting the construction done in [35] for non-delay differential equations. In this purpose, we need to use again the following weighted Banach spaces on $\mathbb{R}$ : the space $\left(\mathscr{C}_{\alpha}(\mathbb{R}, \mathbb{R}),\|\cdot\|_{\alpha}\right)$ of continuous functions on $\mathbb{R}$ bounded in norm $\|\cdot\|_{\alpha}$ and the space

$$
\mathcal{B}_{\delta_{0} / 2}^{\alpha}\left(\mathbb{R}, \mathbb{R}^{n-1}\right)=\left\{\mathrm{f}: \mathbb{R} \rightarrow \mathbb{R}^{n-1} \text { continuous such that: }\|\mathrm{f}\|_{\infty} \leq \delta_{0} / 2, \text { and }\|\mathrm{f}\|_{\alpha}<+\infty\right\},
$$

where $\alpha$ is a strictly positive number (whose range will be specified later in the proof) and $\|\cdot\|_{\alpha}$ stands for the weighted norm by the exponent $\alpha>0$ both for functions with values in $\mathbb{R}$ or with values in $\mathbb{R}^{n}$ (to avoid cumbersome notations).

$\left(\mathcal{B}_{\delta_{0} / 2}^{\alpha}\left(\mathbb{R}, \mathbb{R}^{n-1}\right),\|\cdot\|_{\alpha}\right)$ is a Banach space, and note that the continuous functions over $\mathbb{R}$ bounded in norm $\|\cdot\|_{\alpha}$ are in fact automatically bounded on $\mathbb{R}_{+}$but not on $\mathbb{R}_{-}$. Lastly, we will equip the product space $\mathscr{C}_{\alpha}(\mathbb{R}, \mathbb{R}) \times \mathcal{B}_{\delta_{0} / 2}^{\alpha}\left(\mathbb{R}, \mathbb{R}^{n-1}\right)$ with the norm given by the maximum of the weighted norms of the two components. To avoid useless cumbersome notations again, we will still denote by $\|\cdot\|_{\alpha}$ this norm, i.e we will set:

$$
\left\|\left(\mathrm{f}_{1}, \mathrm{f}_{2}\right)\right\|_{\alpha}:=\max \left(\left\|\mathrm{f}_{1}\right\|_{\alpha},\left\|\mathrm{f}_{2}\right\|_{\alpha}\right) .
$$

Keeping the same choice of $\delta_{0}>0$ and $\eta_{0}>0$ as for Lemma (4.2), (4.1), and (4.3), we set $\eta_{0}^{\prime}=\frac{\eta_{0}}{2}$ and choose $0<\eta<\eta_{0}^{\prime}(\eta$ satisfying therefore $(\star \star))$ and $\tau>0$, and we consider an initial condition $\left(\phi_{1}, y_{1}\right) \in \mathscr{C}([-1,0], \mathbb{R}) \times \mathcal{B}_{\delta_{0} / 2}\left([-1,0], \mathbb{R}^{n-1}\right)$. For simplicity of notations again, we denote by $\left(\psi_{1}(t), y_{1}(t)\right)$ the solution of (3) starting at the initial condition $\left(\phi_{1}, y_{1}\right)$, and taken at time $t \geq 0$, i.e defined by:

$$
\begin{aligned}
\psi_{1}(t) & :=\psi\left(\phi_{1}, y_{1}, t\right) \\
y_{1}(t) & :=y\left(\phi_{1}, y_{1}, t\right) .
\end{aligned}
$$

In order to rewrite Equation (3) in a more concise way, we will also set:

$$
\begin{aligned}
& p\left(\psi_{1}, y_{1}, \psi_{2}, y_{2}\right)=\frac{2 \pi \tau}{T}+\eta \tau \mathrm{g}_{1}\left(\psi_{1}, y_{1}, \psi_{2}, y_{2}\right) \\
& q\left(\psi_{1}, y_{1}, \psi_{2}, y_{2}\right)=\tau \mathrm{h}_{2}\left(\psi_{1}, y_{1}\right)+\eta \tau \mathrm{g}_{2}\left(\psi_{1}, y_{1}, \psi_{2}, y_{2}\right) .
\end{aligned}
$$

(i) By choice of $\eta$ and $\left(\phi_{1}, y_{1}\right)$, we know (see Item (ii) of Remark (3.1)) that we have $\left\|y_{1}(t)\right\| \leq \delta_{0} / 2$ for any $t \geq 0$. It thus comes, by $(\star \star)$ :

$$
\forall t \geq 0,\left\|\dot{y}_{1}(t)-\tau A y_{1}(t)\right\| \leq \tau \mathcal{K}\left(\mathrm{h}_{2}\right)\left(\frac{\delta_{0}}{2}\right)^{2}+\eta \tau \mathcal{K}\left(\mathrm{g}_{2}, \frac{\delta_{0}}{2}\right) .
$$


(ii) Now we pullback the trajectory $\left(\psi_{1}(t), y_{1}(t)\right)_{t \geq 0}$ by setting:

$$
\begin{gathered}
\tilde{\psi}_{1}(t)=\psi_{1}(t+1) \\
\tilde{y}_{1}(t)=y_{1}(t+1),
\end{gathered}
$$

so that the inequality stated in (i) be valid for any $t \geq-1$. Notice the curve $\left(\tilde{\psi}_{1}(t), \tilde{y}_{1}(t)\right)_{t \geq-1}$ is still a trajectory solution of Equation (3), and therefore is continuously differentiable.

Then, we extend this trajectory to a $\mathscr{C}^{1}$ curve $\left(\tilde{\psi}_{1}(t), \tilde{y_{1}}(t)\right)_{t \in \mathbb{R}}$ over the entire real line by imposing the following conditions:

(i) $\left(\tilde{\psi}_{1}(t), \tilde{y}_{1}(t)\right)_{t \in \mathbb{R}}$ satisfies $\sup _{s \leq 0}\left|\tilde{\psi}_{1}(s)\right|<+\infty$

(ii) $\left(\tilde{\psi}_{1}(t), \tilde{y}_{1}(t)\right)_{t \in \mathbb{R}}$ satisfies $\sup _{s \leq 0}\left\|\tilde{y}_{1}(s)\right\|<\delta_{0} / 2$

(iii) For any $s \leq 0,\left\|\dot{\tilde{y}}(s)-\tau A \tilde{y_{1}}(s)\right\| \leq \tau \mathcal{K}\left(\mathrm{h}_{2}\right)\left(\frac{\delta_{0}}{2}\right)^{2}+\eta \tau \mathcal{K}\left(\mathrm{g}_{2}, \frac{\delta_{0}}{2}\right)$

Now, as in the construction of the manifold $\mathcal{M}_{\alpha}$ (see Proposition 4.2 and Lemma 4.3) let us consider the operator $T_{1}\left(\cdot, \cdot, \tilde{\psi_{1}}, \tilde{y_{1}}\right)$ defined by :

$$
\begin{array}{llll}
T_{1}\left(\cdot, \cdot, \tilde{\psi}_{1}, \tilde{y}_{1}\right): & (\gamma, \xi) & \mapsto \quad(\tilde{\gamma}, \tilde{\xi}) & \\
& \mathscr{C}_{\alpha}(\mathbb{R}, \mathbb{R}) \times \mathcal{B}_{\delta_{0} / 2}^{\alpha}\left(\mathbb{R}, \mathbb{R}^{n-1}\right) & \rightarrow \mathscr{C}_{\alpha}(\mathbb{R}, \mathbb{R}) \times \mathcal{B}_{\delta_{0} / 2}^{\alpha}\left(\mathbb{R}, \mathbb{R}^{n-1}\right)
\end{array}
$$

where for any time $t \in \mathbb{R}$ :

$$
\left\{\begin{array}{c}
\tilde{\gamma}(t)=-\int_{t}^{+\infty}\left\{p\left[\tilde{\psi}_{1}(s)+\gamma(s), \tilde{y_{1}}(s)+\xi(s), \tilde{\psi}_{1}(s-1)+\gamma(s-1), \tilde{y}_{1}(s-1)+\xi(s-1)\right]-\dot{\tilde{\psi}}_{1}(s)\right\} d s \\
\tilde{\xi}(t)=\int_{-\infty}^{t} e^{\tau A(t-s)}\left\{q\left[\tilde{\psi}_{1}(s)+\gamma(s), \tilde{y_{1}}(s)+\xi(s), \tilde{\psi}_{1}(s-1)+\gamma(s-1), \tilde{y}_{1}(s-1)+\xi(s-1)\right]\right. \\
\left.-\tilde{\tilde{y}_{1}}(s)+\tau A \tilde{y_{1}}(s)\right\} d s
\end{array}\right.
$$

Let us verify this map is well defined, i.e $(\tilde{\gamma}, \tilde{\xi})$ actually belongs to $\mathscr{C}_{\alpha}(\mathbb{R}, \mathbb{R}) \times \mathcal{B}_{\delta_{0} / 2}^{\alpha}\left(\mathbb{R}, \mathbb{R}^{n-1}\right)$. We verify it for $\tilde{\gamma}$, the similar argument working for $\tilde{\xi}$.

By definition the curve $\left(\tilde{\psi}_{1}(t), \tilde{y}_{1}(t)\right)_{t \geq 0}$ satisfies Equation (3) and so for any time $t \geq 0$ :

$$
\begin{aligned}
\tilde{\gamma}(t)=-\int_{t}^{+\infty} & \left\{p\left[\tilde{\psi}_{1}(s)+\gamma(s), \tilde{y}_{1}(s)+\xi(s), \tilde{\psi}_{1}(s-1)+\gamma(s-1), \tilde{y_{1}}(s-1)+\xi(s-1)\right]\right. \\
& \left.-p\left[\tilde{\psi}_{1}(s), \tilde{y}_{1}(s), \tilde{\psi}_{1}(s-1), \tilde{y}_{1}(s-1)\right]\right\} d s .
\end{aligned}
$$

Thus, we have, for any time $t \geq 0$ :

$$
\begin{aligned}
|\tilde{\gamma}(t)| & \leq \eta \tau \mathcal{K}\left(D \mathrm{~g}_{1}, \delta_{0}\right) \int_{t}^{+\infty} \max [|\gamma(s)|,\|\xi(s)\|,|\gamma(s-1)|,\|\xi(s-1)\|] d s, \\
& \leq \frac{\eta \tau}{\alpha} \mathcal{K}\left(D \mathrm{~g}_{1}, \delta_{0}\right) \cdot\|(\gamma, \xi)\|_{\alpha} e^{-\alpha t},
\end{aligned}
$$

where $\mathcal{K}\left(D \mathrm{~g}_{1}, \delta_{0}\right)=\sup _{\mathfrak{z} \in\left([0,2 \pi] \times \mathcal{W}_{\delta_{0}}\right)^{2}}\left\|D \mathrm{~g}_{1}(\mathfrak{z})\right\|$. This implies (since $\mathcal{K}\left(D \mathrm{~g}_{1}, \delta_{0}\right)$ is bounded independently on $\left.\left(\phi_{1}, y_{1}\right)\right): \sup _{t \geq 0}|\tilde{\gamma}(t)| e^{\alpha t}<+\infty$. Then, for any $t \leq 0$ the quantity:

$$
\left|\int_{t}^{0}\left\{p\left[\tilde{\psi}_{1}(s)+\gamma(s), \tilde{y_{1}}(s)+\xi(s), \tilde{\psi}_{1}(s-1)+\gamma(s-1), \tilde{y_{1}}(s-1)+\xi(s-1)\right]-\dot{\tilde{\psi}}_{1}(s)\right\} d s\right| \cdot e^{\alpha t}
$$

is smaller than:

$$
\left\{\left|\tilde{\psi}_{1}(0)\right|+\left|\tilde{\psi}_{1}(t)\right|+\sup _{s \in[t, 0]}\left|p\left[\tilde{\psi}_{1}(s)+\gamma(s), \tilde{y_{1}}(s)+\xi(s), \tilde{\psi}_{1}(s-1)+\gamma(s-1), \tilde{y_{1}}(s-1)+\xi(s-1)\right]\right| \cdot|t|\right\} \cdot e^{\alpha t} .
$$

Since $(\xi(s))_{s \leq 0}$ is bounded and $\left(\tilde{y}_{1}(s)\right)_{s \leq 0}$ as well (by condition (ii)), we get that the superior bound in the last inequality is bounded uniformly in $t$ : thus (as $\left(\tilde{\psi}_{1}(t)\right)_{t \leq 0}$ is bounded by condition (i)), we obtained

$$
\sup _{t \leq 0}|\tilde{\gamma}(t)| e^{\alpha t}<+\infty
$$

and so $\tilde{\gamma} \in \mathscr{C}_{\alpha}(\mathbb{R}, \mathbb{R})$. Using $(\star)$ and $(\star \star)$, the similar argument works to prove that $\tilde{\xi} \in \mathcal{B}_{\delta_{0} / 2}^{\alpha}\left(\mathbb{R}, \mathbb{R}^{n-1}\right)$, using this time condition (iii). 
Furthermore, for a good choice of $\alpha$ (that we state below), the map $T_{1}\left(\cdot, \cdot, \tilde{\psi}_{1}, \tilde{y}_{1}\right)$ is a contraction. Indeed, let us take two couples $\left(\gamma_{1}, \xi_{1}\right)$ and $\left(\gamma_{2}, \xi_{2}\right)$ in $\mathscr{C}_{\alpha}(\mathbb{R}, \mathbb{R}) \times \mathcal{B}_{\delta_{0} / 2}^{\alpha}\left(\mathbb{R}, \mathbb{R}^{n-1}\right)$ and consider the term:

$$
\left\|T_{1}\left(\gamma_{1}, \xi_{1}, \tilde{\psi}_{1}, \tilde{y}_{1}\right)-T_{1}\left(\gamma_{2}, \xi_{2}, \tilde{\psi}_{1}, \tilde{y_{1}}\right)\right\|_{\alpha}=\max \left(\sup _{t \in \mathbb{R}}\left|\tilde{\gamma_{1}}(t)-\tilde{\gamma_{2}}(t)\right| e^{\alpha t}, \sup _{t \in \mathbb{R}}\left\|\tilde{\xi}_{1}(t)-\tilde{\xi}_{2}(t)\right\| e^{\alpha t}\right) .
$$

We have, for any time $t$ in $\mathbb{R}$ :

$$
\begin{aligned}
\left|\tilde{\gamma}_{1}(t)-\tilde{\gamma}_{2}(t)\right| \leq \int_{t}^{+\infty} & \mid p\left[\tilde{\psi}_{1}(s)+\gamma_{1}(s), \tilde{y_{1}}(s)+\xi_{1}(s), \tilde{\psi}_{1}(s-1)+\gamma_{1}(s-1), \tilde{y_{1}}(s-1)+\xi_{1}(s-1)\right] \\
& -p\left[\tilde{\psi}_{1}(s)+\gamma_{2}(s), \tilde{y_{1}}(s)+\xi_{2}(s), \tilde{\psi_{1}}(s-1)+\gamma_{2}(s-1), \tilde{y_{1}}(s-1)+\xi_{2}(s-1)\right] \mid d s
\end{aligned}
$$

which as above yields:

$$
\left\|\tilde{\gamma_{1}}-\tilde{\gamma_{2}}\right\|_{\alpha} \leq \frac{\eta \tau}{\alpha} \mathcal{K}\left(D g_{1}, \delta_{0}\right)\left\|\left(\gamma_{1}-\gamma_{2}, \xi_{1}-\xi_{2}\right)\right\|_{\alpha}
$$

A similar inequality is obtained by the same reasoning for $\left\|\tilde{\xi}_{1}-\tilde{\xi}_{2}\right\|_{\alpha}$. Namely:

$$
\left\|\tilde{\xi}_{1}-\tilde{\xi}_{2}\right\|_{\alpha} \leq C \cdot \frac{\tau \mathcal{K}\left(D \mathrm{~h}_{2}\right) \delta_{0}+\eta \tau \mathcal{K}\left(D \mathrm{~g}_{2}, \delta_{0}\right)}{-\tau \sigma-\alpha}\left\|\left(\gamma_{1}-\gamma_{2}, \xi_{1}-\xi_{2}\right)\right\|_{\alpha}
$$

Thus, choosing $\alpha_{1}$ such that:

$$
\eta \tau \mathcal{K}\left(D \mathrm{~g}_{1}, \delta_{0}\right)<\alpha_{1}<2 \alpha_{1}<-\tau \sigma
$$

(in this way $\alpha_{1}$ depends only on $\eta$ and $\tau$, since $\mathcal{K}\left(D \mathrm{~g}_{1}, \delta_{0}\right)$ does not depend on $\left.\left(\phi_{1}, y_{1}\right)\right)$ we obtain:

$$
\left\|\tilde{\xi}_{1}-\tilde{\xi}_{2}\right\|_{\alpha_{1}} \leq 2 C \cdot \frac{\mathcal{K}\left(D \mathrm{~h}_{2}\right) \delta_{0}+\eta \mathcal{K}\left(D \mathrm{~g}_{2}, \delta_{0}\right)}{-\sigma}\left\|\left(\gamma_{1}-\gamma_{2}, \xi_{1}-\xi_{2}\right)\right\|_{\alpha_{1}}
$$

which proves, by condition $(\star \star)$, that $T_{1}\left(\cdot, \cdot, \tilde{\psi}_{1}, \tilde{y_{1}}\right)$ is a contraction.

(iii) Finally, by the Banach contractive mapping theorem, there exists a unique fixed point $\left(\gamma_{1}, \xi_{1}\right)$ of the map $T_{1}\left(\cdot, \cdot, \tilde{\psi}_{1}, \tilde{y_{1}}\right)$. We then set:

$$
\begin{aligned}
\psi_{0} & :=\tilde{\psi}_{1}+\gamma_{1} \\
y_{0} & :=\tilde{y}_{1}+\xi_{1},
\end{aligned}
$$

which are curves defined and continuously differentiable over the entire real line $\mathbb{R}$.

By construction, $\left\|y_{0}(s)\right\| \leq \delta_{0}$ for any time $s \leq 0$, and we have, for any $t \in \mathbb{R}$ :

$$
\begin{aligned}
& \dot{\psi_{0}}(t)=p\left[\psi_{0}(t), y_{0}(t), \psi_{0}(t-1), y_{0}(t-1)\right] \\
& \dot{y}_{0}(t)=\tau A y_{0}+q\left[\psi_{0}(t), y_{0}(t), \psi_{0}(t-1), y_{0}(t-1)\right],
\end{aligned}
$$

and thus, at any given time $s \in[-1,0]$ :

$$
\forall t \geq-s, \mathcal{T}_{t, \eta, \tau}\left(\psi_{0 \mid \mathbb{R}_{-}}, y_{0 \mid \mathbb{R}_{-}}\right)(s)=\left(\psi_{0}(t+s), y_{0}(t+s)\right) .
$$

This means that $\left(\psi_{0 \mid \mathbb{R}_{+}}, y_{0 \mid \mathbb{R}_{+}}\right)$is the solution of the delay equation starting at the initial condition $\psi_{0 \mid \mathbb{R}_{-}}, y_{0 \mid \mathbb{R}_{-}}$ (and even starting at $\psi_{0 \mid[-1,0]}, y_{0 \mid[-1,0]}$ ). Now, at any fixed time $s \in[-1,0]$ we have, for any $t \geq-s$ :

$$
\begin{aligned}
\left\|\mathcal{T}_{t, \eta, \tau}\left(\psi_{0 \mid \mathbb{R}_{-}}, y_{0 \mid \mathbb{R}_{-}}\right)(s)-\mathcal{T}_{t+1, \eta, \tau}\left(\phi_{1}, y_{1}\right)(s)\right\| & =\left(\left|\psi_{0}(t+s)-\psi_{1}(t+s+1)\right|,\left\|y_{0}(t+s)-y_{1}(t+s+1)\right\|\right) \\
& =\left(\left|\psi_{0}(t+s)-\tilde{\psi}_{1}(t+s)\right|, \| y_{0}(t+s)-\tilde{y_{1}}(t+s)||\right) \\
& =\left(\left|\gamma_{1}(t+s)\right|,|| \xi_{1}(t+s) \|\right)
\end{aligned}
$$

and so:

$$
\lim _{t \rightarrow \infty} \sup _{s \in[-1,0]}\left\|\mathcal{T}_{t, \eta, \tau}\left(\psi_{0 \mid \mathbb{R}_{-}}, y_{0 \mid \mathbb{R}_{-}}\right)(s)-\mathcal{T}_{t+1, \eta, \tau}\left(\phi_{1}, y_{1}\right)(s)\right\|=0
$$

this pointwise convergence being exponentially fast.

To conclude, since the curve $\left(y_{0}(t)\right)_{t \leq 0}$ is the solution of the equation:

$$
\dot{z}(t)=\tau A z+q\left[\psi_{0 \mid \mathbb{R}_{-}}(t), z(t), \psi_{0 \mid \mathbb{R}_{-}}(t-1), z(t-1)\right], \quad t \leq 0
$$


we have that $\left(\psi_{0 \mid \mathbb{R}_{-}}, y_{0 \mid \mathbb{R}_{-}}\right)$belongs to the manifold $\mathcal{M}_{\alpha}$, as desired.

\subsection{Proof of Proposition 5.2 (a coordinate transformation for Equation (9)).}

Proof. (i) The map $\mathbb{T}_{1}$ is of the form: $\mathbb{T}_{1}(\beta, \phi)=(\beta, \phi)-\eta \cdot\left(0, \mathrm{Q}\left[\beta, \phi, \phi_{-1}\right]\right)$, with:

$$
\forall s \leq 0, \mathrm{Q}[\beta, \phi, \phi(s-1)]:=\Re\left(\sum_{(m \neq n) \in \mathbb{Z} \times \mathbb{Z}} \frac{a_{m, n}}{2 i \pi \frac{1}{T}(m-n)} e^{i\left(m \phi(s)-n \phi(s-1)+2 \pi n \frac{\tau}{T}\right)}\left(e^{i(m-n) \beta(s)}-1\right)\right),
$$

(where we recall that the notation $\phi_{-1}$ stands for the function $\phi_{-1}: s \mapsto \phi(s-1)$, with $s \in \mathbb{R}_{-}$). Given $\left(\beta_{1}, \phi_{1}\right)$ and $\left(\beta_{2}, \phi_{2}\right)$ in $\mathscr{C}_{\alpha}\left(\mathbb{R}_{-}, \mathbb{R}\right)^{2}$, we have, for any $s \leq 0$ :

$$
\left|\mathrm{Q}\left[\beta_{1}, \phi_{1}, \phi_{1}(s-1)\right]-\mathrm{Q}\left[\beta_{2}, \phi_{2}, \phi_{2}(s-1)\right]\right| \leq \sum_{(m \neq n) \in \mathbb{Z} \times \mathbb{Z}} \frac{\left|\Re\left(a_{m, n}\right)\right|+\left|\Im\left(a_{m, n}\right)\right|}{\left|2 \pi \frac{1}{T}(m-n)\right|}\left(2\left|X_{1}-X_{2}\right|+\left|Y_{1}-Y_{2}\right|\right),
$$

where:

$$
\left\{\begin{array}{l}
X_{1}=m \phi_{1}(s)-n \phi_{1}(s-1)+2 \pi \frac{n \tau}{T} \\
Y_{1}=(m-n) \beta_{1}(s)
\end{array}\right.
$$

and similarly for $X_{2}, Y_{2}$.

Since the function $\mathrm{h}$ is $\mathscr{C}^{2}$, therefore the following series:

$$
\sum_{(m \neq n) \in \mathbb{Z} \times \mathbb{Z}} \frac{\left|\Re\left(a_{m, n}\right)\right|+\left|\Im\left(a_{m, n}\right)\right|}{\left|2 \pi \frac{1}{T}(m-n)\right|}(|m|+|n|)
$$

converges. This gives that the map $(\beta, \phi) \mapsto\left(0, \mathrm{Q}\left[\beta, \phi, \phi_{-1}\right]\right)$ is a Lipschitz map from $\mathscr{C}_{\alpha}\left(\mathbb{R}_{-}, \mathbb{R}^{2}\right.$ to $\mathscr{C}_{\alpha}\left(\mathbb{R}_{-}, \mathbb{R}\right)^{2}$. As a Lipschitz perturbation of the identity map on a Banach space, the map $\mathbb{T}_{1}$ is therefore a bi-Lipschitz homeomorphism.

(ii) Let $(\beta, \phi)$ and $(\mathrm{h}, \mathrm{k})$ in $\mathscr{C}_{\alpha}\left(\mathbb{R}_{-}, \mathbb{R}\right)^{2}$. Given $s \leq 0$, the difference:

$$
-\left(\mathbb{T}_{2}(\beta+\mathrm{h}, \phi+\mathrm{k})(s)-\mathbb{T}_{2}(\beta, \phi)(s)-(\mathrm{h}(s), \mathrm{k}(s))\right.
$$

is equal to

$$
\begin{array}{r}
\eta\left(0, \sum_{(m \neq n) \in \mathbb{Z} \times \mathbb{Z}} \frac{\Re\left(a_{m, n}\right)}{2 \pi \frac{1}{T}(m-n)}\left[\sin \left(X_{\phi}+X_{\mathrm{k}}+Y_{\beta}+Y_{\mathrm{h}}\right)-\sin \left(X_{\phi}+X_{\mathrm{k}}\right)-\sin \left(X_{\phi}+Y_{\beta}\right)+\sin \left(X_{\phi}\right)\right]\right) \\
+\eta\left(0, \sum_{(m \neq n) \in \mathbb{Z} \times \mathbb{Z}} \frac{\Im\left(a_{m, n}\right)}{2 \pi \frac{1}{T}(m-n)}\left[\cos \left(X_{\phi}+X_{\mathrm{k}}+Y_{\beta}+Y_{\mathrm{h}}\right)-\cos \left(X_{\phi}+X_{\mathrm{k}}\right)-\cos \left(X_{\phi}+Y_{\beta}\right)+\cos \left(X_{\phi}\right)\right]\right)
\end{array}
$$

where we have set:

$$
\left\{\begin{array}{l}
X_{\phi}=m \phi(s)-n \phi(s-1)+2 \pi n \frac{\tau}{T} \\
X_{\mathrm{k}}=m \mathrm{k}(s)-n \mathrm{k}(s-1) \\
Y_{\beta}=(m-n) \beta(s) \\
Y_{\mathrm{h}}=(m-n) \mathrm{h}(s)
\end{array} .\right.
$$

Let's look at the first sum of this term, expressed by the function sin and $\Re\left(a_{m, n}\right)$. We have:

$$
\begin{aligned}
& {\left[\sin \left(X_{\phi}+X_{\mathrm{k}}+Y_{\beta}+Y_{\mathrm{h}}\right)-\sin \left(X_{\phi}+X_{\mathrm{k}}\right)-\sin \left(X_{\phi}+Y_{\beta}\right)+\sin \left(X_{\phi}\right)\right] } \\
= & \sin \left(X_{\phi}+Y_{\beta}\right)\left[\cos \left(X_{\mathrm{k}}+Y_{\mathrm{h}}\right)-1\right]-\sin \left(X_{\phi}\right)\left[\cos \left(X_{\mathrm{k}}\right)-1\right]+\sin \left(X_{\mathrm{k}}+Y_{\mathrm{h}}\right) \cos \left(X_{\phi}+Y_{\beta}\right)-\sin \left(X_{\mathrm{k}}\right) \cos \left(X_{\phi}\right) \\
= & \left(X_{\mathrm{k}}+Y_{\mathrm{h}}\right) \cos \left(X_{\phi}+Y_{\beta}\right)-X_{\mathrm{k}} \cos \left(X_{\phi}\right)+Z_{1, \mathbf{k}, \mathbf{h}}
\end{aligned}
$$

where $\boldsymbol{Z}_{\mathbf{1}, \mathbf{k}, \mathbf{h}}$ satisfies: $\sup _{s \geq 0}\left|\boldsymbol{Z}_{\mathbf{1 , \mathbf { k } , \mathbf { h }}}\right| e^{2 \alpha s}=o\left(\|(\mathrm{~h}, \mathrm{k})\|_{\alpha}\right)$ when $\|(\mathrm{h}, \mathrm{k})\|_{\alpha}$ tends to 0. Similarly we have:

$$
\begin{aligned}
& {\left[\cos \left(X_{\phi}+X_{\mathrm{k}}+Y_{\beta}+Y_{\mathrm{h}}\right)-\cos \left(X_{\phi}+X_{\mathrm{k}}\right)-\cos \left(X_{\phi}+Y_{\beta}\right)+\cos \left(X_{\phi}\right)\right] } \\
= & -\left(X_{\mathrm{k}}+Y_{\mathrm{h}}\right) \sin \left(X_{\phi}+Y_{\beta}\right)+X_{\mathrm{k}} \sin \left(X_{\phi}\right)+Z_{\mathbf{2}, \mathbf{k}, \mathbf{h}}
\end{aligned}
$$

where $\boldsymbol{Z}_{\mathbf{2}, \mathbf{k}, \mathbf{h}}$ satisfies: $\sup _{s \geq 0}\left|\boldsymbol{Z}_{\mathbf{2}, \mathbf{k}, \mathbf{h}}\right| e^{2 \alpha s}=o\left(\|(\mathrm{~h}, \mathrm{k})\|_{\alpha}\right)$ when $\|(\mathrm{h}, \mathrm{k})\|_{\alpha}$ tends to 0 . This gives Item (ii). 
(iii) Let us fix $\left(\beta, \phi^{\text {new }}\right) \in \mathscr{C}_{\alpha}\left(\mathbb{R}_{-}, \mathbb{R}\right)^{2}$. As said in Item (i), the map $\mathbb{T}_{1}$ is a Lipschitz perturbation of the identity map on $\mathscr{C}_{\alpha}\left(\mathbb{R}_{-}, \mathbb{R}\right)^{2}$ : thus $\mathbb{T}_{1}^{-1}\left(\beta, \phi^{\text {new }}\right)$ can be obtained as the limit of the sequence $\left(Z_{n}\right)_{n \geq 0}$ defined by the relations:

$$
\left\{\begin{array}{ll}
Z_{0} & =\left(\beta_{0}, \phi_{0}^{\text {new }}\right) \\
Z_{n+1} & =\mathrm{F}\left(Z_{n}\right),
\end{array} \quad n \geq 0\right.
$$

where the point $\left(\beta_{0}, \phi_{0}^{\text {new }}\right)$ is taken arbitrarily in $\mathscr{C}_{\alpha}\left(\mathbb{R}_{-}, \mathbb{R}\right)^{2}$, and the map $\mathrm{F}$ is defined by the relation $\mathrm{F}(X, Y)=\eta \mathcal{Q}\left(X, Y, Y_{-1}\right)+\left(\beta, \phi^{\text {new }}\right)$, for any $(X, Y) \in \mathscr{C}_{\alpha}\left(\mathbb{R}_{-}, \mathbb{R}\right)^{2}$. Since $\mathbb{T}_{2}$ is $\mathscr{C}^{1}$, this gives:

$$
D \mathbb{T}_{[-1]}\left(\beta, \phi^{\text {new }}\right)(\mathrm{h}, \mathrm{k})=(\mathrm{h}, \mathrm{k})+\eta D \mathcal{Q}\left(\mathbb{T}_{[-1]}\left(\beta, \phi^{\text {new }}\right), \mathbb{T}_{[-1]}\left(\beta, \phi^{\text {new }}\right)_{2}(\cdot-1)\right)(\mathrm{h}, \mathrm{k})+o\left(\|(\mathrm{~h}, \mathrm{k})\|_{\alpha}\right)
$$

when $\|(\mathrm{h}, \mathrm{k})\|_{\alpha}$ tends to 0 , where we have denoted by $\mathbb{T}_{[-1]}\left(\beta, \phi^{\text {new }}\right)_{2}$ the second coordinate of $\mathbb{T}_{[-1]}\left(\beta, \phi^{\text {new }}\right)$ (and where, as before, $\mathbb{T}_{[-1]}\left(\beta, \phi^{\text {new }}\right)_{2}(\cdot-1)$ stands for the map $s \mapsto \mathbb{T}_{[-1]}\left(\beta, \phi^{\text {new }}\right)_{2}(s-1)$ ). By Item (ii), the linear map $D \mathcal{Q}\left(\mathbb{T}_{[-1]}\left(\beta, \phi^{\text {new }}\right), \mathbb{T}_{[-1]}\left(\beta, \phi^{\text {new }}\right)_{2}(\cdot-1)\right)$ is continuous. Hence the result.

\section{REFERENCES}

[1] Isam Al-Darabsah and Sue Campbell. A phase model with large time delayed coupling. Physica D: Nonlinear Phenomena, page 132559, 052020.

[2] Stefan Banach. Sur les opérations dans les ensembles abstraits et leur application aux équations intégrales. Fundamenta Mathematicae, 3(1):133-181, 1922.

[3] Peter W. Bates, Kening Lu, and Chongchun Zeng. Existence and persistence of invariant manifolds for semiflows in banach space. Memoirs of the American Mathematical Society, 135(645):0-0, 1998.

[4] Peter W. Bates, Kening Lu, and Chongchun Zeng. Persistence of overflowing manifolds for semiflow. Communications on Pure and Applied Mathematics, 52(8):983-1046, 1999.

[5] Peter W. Bates, Kening Lu, and Chongchun Zeng. Approximately invariant manifolds and global dynamics of spike states. Inventiones mathematicae, 174(645):355-433, 2008.

[6] G. Brown, C.M. Postlethwaite, and M. Silber. Time-delayed feedback control of unstable periodic orbits near a subcritical hopf bifurcation. Physica D: Nonlinear Phenomena, 240(9):859 - 871, 2011.

[7] X.-Y. Chen, J.K. Hale, and B. Tan. Synchronization is enhanced in weighted complex networks. Journal of Differential Equations, 139:283-318, 1997.

[8] R. Cooke and V.I. Arnold. Ordinary Differential Equations. Springer Textbook. Springer Berlin Heidelberg, 1992.

[9] E. Cotton. Sur les solutions asymptotiques des équations différentielles. Ann. de l'Éc. Norm., 28(1911):473-521, 1911.

[10] Thomas Dahms, Philipp Hövel, and Eckehard Schöll. Stabilizing continuous-wave output in semiconductor lasers by timedelayed feedback. Phys. Rev. E, 78:056213, Nov 2008.

[11] Jaap Eldering. Normally hyperbolic invariant manifolds, volume 2 of Atlantis Studies in Dynamical Systems. Atlantis Press, Paris, 2013. The noncompact case.

[12] Neil Fenichel. Persistence and smoothness of invariant manifolds for flows. Indiana Univ. Math. J., 21:193-226, 1972.

[13] B. Fiedler, V. Flunkert, M. Georgi, P. Hövel, and E. Schöll. Refuting the odd-number limitation of time-delayed feedback control. Phys. Rev. Lett., 98:114101, Mar 2007.

[14] B. Fiedler, S. Yanchuk, V. Flunkert, P. Hövel, H.-J. Wünsche, and E. Schöll. Delay stabilization of rotating waves near fold bifurcation and application to all-optical control of a semiconductor laser. Phys. Rev. E, 77:066207, Jun 2008.

[15] Valentin Flunkert and Eckehard Schöll. Suppressing noise-induced intensity pulsations in semiconductor lasers by means of time-delayed feedback. Phys. Rev. E, 76:066202, Dec 2007.

[16] Jack K. Hale and Marion Weedermann. On perturbations of delay-differential equations with periodic orbits. Journal of Differential Equations, 197(2):219 - 246, 2004.

[17] J.K. Hale. Ordinary Differential Equations. Pure and applied mathematics : a series of texts and monographs. WileyInterscience, 1969.

[18] J.K. Hale and S.M.V. Lunel. Introduction to Functional Differential Equations. Number vol. 99 in Applied mathematical sciences. Springer-Verlag, 1993.

[19] M.W. Hirsch, C.C. Pugh, and M. Shub. Invariant Manifolds. Lecture Notes in Mathematics. Springer Berlin Heidelberg, 2006.

[20] P. Hövel and E. Schöll. Control of unstable steady states by time-delayed feedback methods. Phys. Rev. E, 72:046203, Oct 2005.

[21] Philipp Hövel, Markus A. Dahlem, and Eckehard Schöll. Control of synchronization in coupled neural systems by timedelayed feedback. Int. J. Bifurc. Chaos, 20:813-825, 2010.

[22] Eugene M. Izhikevich. Phase models with explicit time delays. Phys. Rev. E, 58:905-908, Jul 1998.

[23] R. Lang and K. Kobayashi. External optical feedback effects on semiconductor injection laser properties. IEEE Journal of Quantum Electronics, 16(3):347-355, 1980.

[24] Ricardo Mañé. Persistent manifolds are normally hyperbolic. Trans. Amer. Math. Soc., 246:261-283, 1978. 
[25] A. Miele. The 1st john v. breakwell memorial lecture: Recent advances in the optimization and guidance of aeroassisted orbital transfers. Acta Astronautica, 38:747-768, 1996.

[26] Paul Miller and Jonathan Cannon. Combined mechanisms of neural firing rate homeostasis. Biological Cybernetics, 113:4759, 2019.

[27] Edward Ott, Celso Grebogi, and James A. Yorke. Controlling chaos. Phys. Rev. Lett., 64:1196-1199, Mar 1990.

[28] P. Perlikowski and S. Yanchuk. Delay and periodicity. Phys. Rev. E, 79:046221, Apr 2009.

[29] O. Perron. über stabilität und asymptotisches verhaltender integrale von differentialgleichungssystemen. Math. Z, 29:129$160,1929$.

[30] O. Perron. Die stabilitätsfrage bei differentialgleichungen. Math. Z, 32:703-728, 1930.

[31] Camille Poignard. Inducing chaos in a gene regulatory network by coupling an oscillating dynamics with a hysteresis-type one. Journal of Mathematical Biology, pages 1-34, 2013.

[32] K. Pyragas. Continuous control of chaos by self-controlling feedback. Physics Letters A, 170(6):421 - 428, 1992.

[33] G. Rigatos and P. Siano. A nonlinear optimal control approach of insulin infusion for blood glucose levels regulation. Intelligent Industrial Systems, 3:91-102, 2017.

[34] Michael Rosenblum and Arkady Pikovsky. Delayed feedback control of collective synchrony: An approach to suppression of pathological brain rhythms. Phys. Rev. E, 70:041904, Oct 2004.

[35] Leonid P. Shilnikov, Andrey L. Shilnikov, Dmitry V. Turaev, and Leon O. Chua. Methods of qualitative theory in nonlinear dynamics. Part I. World Scientific, 2001.

[36] Leonid P. Shilnikov, Andrey L. Shilnikov, Dmitry V. Turaev, and Leon O. Chua. Methods of qualitative theory in nonlinear dynamics. Part II. World Scientific, 2001.

[37] J. Sieber. Generic stabilizability for time-delayed feedback control. Proceedings of the Royal Society of London A: Mathematical, Physical and Engineering Sciences, 472(2189), 2016.

[38] Jan Sieber, Matthias Wolfrum, Mark Lichtner, and Serhiy Yanchuk. On the stability of periodic orbits in delay equations with large delay. Discrete and Continuous Dynamical Systems - A, 33:3109, 2013.

[39] Le-Zhi Wang, Ri-Qi Su, Zi-Gang Huang, Xiao Wang, Wen-Xu Wang, Celso Grebogi, and Ying-Cheng Lai. A geometrical approach to control and controllability of nonlinear dynamical networks. Nature Communications, 7(1):11323, 2016.

[40] S.M. Wieczorek, B. Krauskopf, T.B. Simpson, and D. Lenstra. The dynamical complexity of optically injected semiconductor lasers. Physics Reports, 416 (1-2):1 - 128, September 2005. Publisher: Elsevier Science BV.

[41] S. Wiggins, G. Haller, and I. Mezic. Normally Hyperbolic Invariant Manifolds in Dynamical Systems. Applied Mathematical Sciences. Springer New York, 2013.

[42] Serhiy Yanchuk and Matthias Wolfrum. A Multiple Time Scale Approach to the Stability of External Cavity Modes in the Lang-Kobayashi System Using the Limit of Large Delay. SIAM Journal on Applied Dynamical Systems, 9:519-535, January 2010.

[43] Serhiy Yanchuk, Matthias Wolfrum, Philipp Hövel, and Eckehard Schöll. Control of unstable steady states by long delay feedback. Phys. Rev. E, 74:026201, Aug 2006. 